\title{
Representations of the Loop Braid Group and Aharonov-Bohm like effects in discrete $(3+1)$-dimensional higher gauge theory
}

\author{
Alex Bullivant, João Faria Martins ${ }^{\dagger}$ Paul Martin. ${ }^{\ddagger}$ \\ School of Mathematics, University of Leeds, Leeds, LS2 9JT, United Kingdom
}

December 20, 2018

We show that representations of the loop braid group arise from Aharonov-Bohm like effects in finite 2 -group $(3+1)$-dimensional topological higher gauge theory. For this we introduce a minimal categorification of biracks, which we call W-bikoids (welded bikoids). Our main example of W-bikoids arises from finite 2-groups, realised as crossed modules of groups. Given a W-bikoid, and hence a groupoid of symmetries, we construct a family of unitary representations of the loop braid group derived from representations of the groupoid algebra. We thus give a candidate for higher Bais' flux metamorphosis, and hence also a version of a 'higher quantum group'.

Keywords: Loop braid group, virtual braid group, welded braid group, welded knot, mapping class groups, knotted surfaces, categorification, higher gauge theory, flux metamorphosis, topological phases of matter in $3+1 \mathrm{D}$, loop excitations, topological quantum computing, weak Hopf algebras, biracks and biquandles

\section{Introduction}

The motivation for this work is to describe the observable collective properties of loop-like quasiparticles [1, 2, 3, 4, 5, 6] arising in (3+1)-dimensional topological phases of matter with higher gauge symmetry [7, 8, 9, 10, thereby modelling corresponding higher Aharonov-Bohm effects.

Higher gauge theory is a version of gauge theory that features 2-dimensional holonomies along surfaces [11, 12, hence in particular along trajectories of loops moving in 3-dimensional space. In this paper we model Aharonov-Bohm phases that are conjecturaly related to the 2-dimensional holonomies along the surfaces traced by loop-particles as they move.

In a topological system the observable collective properties of particles are, at most, their braidings. The braiding of (unknotted and unlinked) oriented circles is described by the $n$-loop braid group $\mathrm{LB}_{n}$ [13, 14]. So we study representations of $\mathrm{LB}_{n}$. Technically this group can be realised as the mapping class group $\operatorname{MCG}\left(D^{3}, C_{n}\right)$ of self-mappings of the 3 -disk $D^{3}$ that are the identity on $\partial D^{3}$ and fix $C_{n}$, an unlinked union of unknotted oriented circles, setwise, in addition preserving the orientation on the circles. (We review the definitions in §3.4) The group $\mathrm{LB}_{n}$ can also be described in terms of loop motions [13, 15]; see [14, §2].

Let us give a visualization of motions of loops in $(3+1)$-dimensions that would be expected to induce 'Aharonov-Bohm like' effects [3, 4, 5, 6, 16. It will be helpful (cf. [13, 14]) first to work with two circular and parallel loops, and in a frame in which one of the loops is fixed. Thus for example consider two loops that are initially coaxial (as on the left in (1) below), and work in a frame in which the upper loop is fixed. We can then visualize a motion by showing the 'Dirac sheet', the surface swept out by the other loop in this

\footnotetext{
*E-mail address: a.l.bullivant@leeds.ac.uk

$\dagger$ E-mail address: j.fariamartins@leeds.ac.uk

‡E-mail address: p.p.martin@leeds.ac.uk
} 
frame. At times $t=0,1,2$ we might then have:
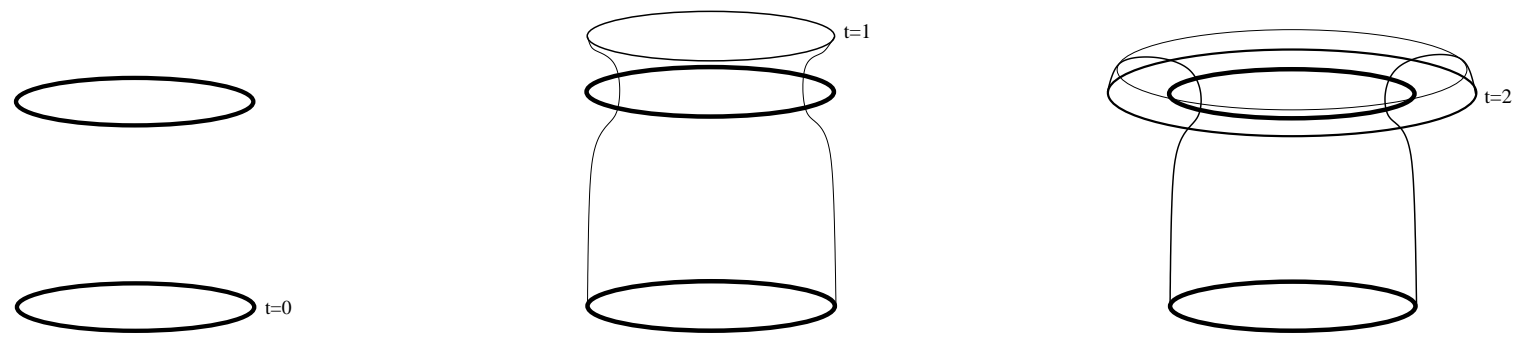

This is analogous to tracking the world-line of a point-particle moving around another point in a plane. Indeed our particular motion is reproduced by rotating this plane into 3 dimensions about a suitable axis in the plane (which axis then becomes the coaxis of the two loops). Note that our motion up to $t=1$ can be completed to a pure loop-braid in two topologically distinct ways, that are both distinct from the 'un-motion'. Firstly the 'moving' loop can return to its original position first as at $t=2$, and from there in the obvious direct way, as shown in figure (2) below.

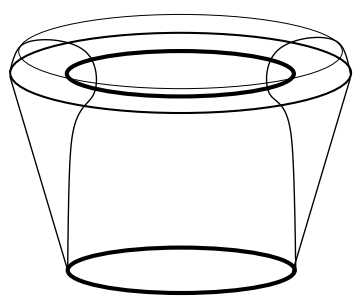

Secondly from $t=1$ we can then break the axial symmetry and move to some position high on the left, say, then return to base in the obvious way from there; see figure (3) below.
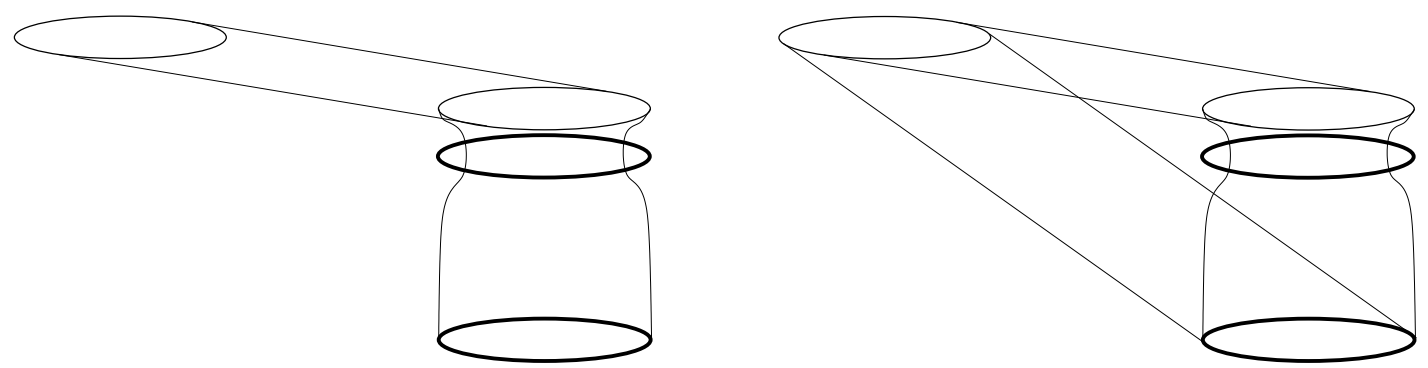

These two topologically distint motions of a loop around another one play a key role in understanding Aharonov-Bohm like effects for loop-particles; see 22.3 .

Let $\sigma$ denote the motion class represented by $t=0 \rightarrow 1$ in (10); and $\nu$ the class represented by $t=1 \rightarrow 3$ in (3). (Keep in mind the moving frame - the loops are exchanged by $\sigma$ and $\nu$.) Extending to a stack of $n$ loops, it turns out that these motions generate $\mathrm{LB}_{n}$. Thus given (I) a corresponding $n$-loop-particle Hilbert space $\mathcal{H}$, in some topological theory, and (II) transformations on $\mathcal{H}$ corresponding to these motions, we will have a representation of the loop braid group $\mathrm{LB}_{n}$. So far there is no higher analogue of the solenoid/electron holography setup or experiments telling us how to obtain (I,II). But there are formal recipes coming from higher gauge theory - as we shall show in $\$ 2$ Since these recipes are necessarily partly ad hoc, we then rely on combinatorial methods to verify the well-definedness Theorem for the representations of $\mathrm{LB}_{n}$, which takes up the remainder of the paper.

Writing $\sigma_{i}$ for the $\sigma$ motion exchanging loops $i$ and $i+1$ it is easy to verify that the $\sigma$ and $\nu$ generators obey braid relations: $\sigma_{i} \sigma_{i+1} \sigma_{i}=\sigma_{i+1} \sigma_{i} \sigma_{i+1}$, and certain mixed braid relations between them. These relations give a homomorphism with a group defined by generators and relations. For the combinatorial proof we will use the isomorphism [13, 14] of $\mathrm{LB}_{n}$ to the welded braid group $\mathrm{WB}_{n}$ [17, 18, 19. This presentation arises in virtual knot theory. In virtual knot theory we have 'virtual' knot and braid diagrams [14, 18, 20, 19, see 
33.3. These diagrams have positive, negative and virtual crossings between strands, as below:

positive crossing

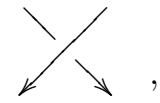

negative crossing

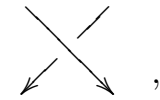

virtual crossing

[1.1] The $n$-strand virtual braid group $\mathrm{VB}_{n}$ (discussed in 93.3 ) is generated, as a monoid, by the elements $S_{a}^{+}[n], S_{a}^{-}[n]$ and $V_{a}[n]$, where $a \in\{1, \ldots, n-1\}$, subject to certain relations; see $\{3.3$. These generators may be depicted as $n$-strand virtual braid diagrams, as below, with composition via vertical stacking.

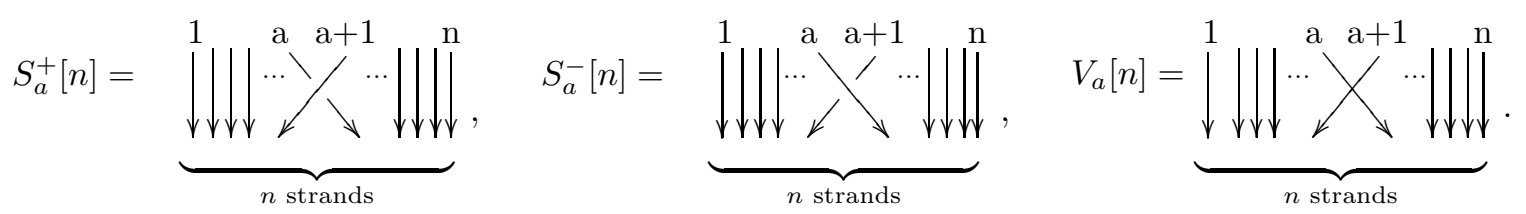

The relations in $\mathrm{VB}_{n}$ between generators are derived from the usual classical, virtual and mixed Reidemeister moves between virtual braid diagrams [18, 19, 20, 21; see equations (44)-(48), and the paragraph below. One passes from $\mathrm{VB}_{n}$ to $\mathrm{WB}_{n}$ by furthermore imposing the the so-called "Forbidden Move F " [19, 21, 22, here called the $W$-move [18]", a short for welded move, displayed in Equation (49).

On generators, the isomorphism $\mathrm{WB}_{n} \rightarrow \mathrm{LB}_{n}$ is given by the motion in Fig. 5 .

[1.2] We have categories $\mathcal{V B}$ and $\mathcal{W B}$ of virtual and welded braid groups. The set of objects of both is $\mathbb{N}$, and $\operatorname{hom}(n, n)$ are $\mathrm{VB}_{n}$ and $\mathrm{WB}_{n}$, respectively. (There are no morphisms $n \rightarrow m$ if $n \neq m$.) The composition $\otimes$ of diagrams through horizontal stacking gives that $\mathcal{V B}$ and $\mathcal{W B}$ are monoidal categories. Note that $S_{2}^{+}[3]=1_{1} \otimes S_{1}^{+}[2], S_{2}^{+}[3]=S_{1}^{+}[2] \otimes 1_{1}$ and so on. Here $1_{1}$ is the identity morphism $1 \rightarrow 1$.

The Reidemeister III-move (46) in $\mathcal{V B}$ and $\mathcal{W B}$, is a consequence of the monoidal category structure in $\mathcal{V B}$ and $\mathcal{W B}$, and the particular case $S_{1}^{+}[3] S_{2}^{+}[3] S_{1}^{+}[3]=S_{2}^{+}[3] S_{1}^{+}[3] S_{2}^{+}[3]$, which can be written as:

$$
\left(S_{1}^{+}[2] \otimes 1_{1}\right)\left(1_{1} \otimes S_{1}^{+}[2]\right)\left(S_{1}^{+}[2] \otimes 1_{1}\right)=\left(1_{1} \otimes S_{1}^{+}[2]\right)\left(S_{1}^{+}[2] \otimes 1_{1}\right)\left(1_{1} \otimes S_{1}^{+}[2]\right) .
$$

And the welded-move in $\mathcal{W B}$ is a consequence of the monoidal structure and the particular case:

$$
\left(V_{1}[2] \otimes 1_{1}\right)\left(1_{1} \otimes S_{1}^{+}[2]\right)\left(S_{1}^{+}[2] \otimes 1_{1}\right)=\left(1_{1} \otimes S_{1}^{+}[2]\right)\left(S_{1}^{+}[2] \otimes 1_{1}\right)\left(1_{1} \otimes V_{1}[2]\right) .
$$

[1.3] One can show (a proof is in e.g. [23, Thm. 2]) that the category $\mathcal{V B}$ of virtual braid groups contains the braid category $\mathcal{B}$ (defined in e.g. in [24, §XIII.2]) as a subcategory — it is the part monoidally generated in $\mathcal{V B}$ by the $S_{1}^{ \pm}[2]$. Suppose we have a monoidal representation of $\mathcal{B}$ in a symmetric category; the main example to have in mind is the category of vector spaces. This lifts automatically 25 to a representation of $\mathcal{V B}$ by mapping $V_{1}[2]$ to the transposition of tensor factors. However, for representations of the loop braid group we require the welded braid group, and in this the welded move (6) is also satisfied. The satisfaction of the welded move is one key outcome of our higher gauge construction.

In order to formalise the representations of $\mathrm{LB}_{n}$ derived from finite 2-group higher gauge theory 11 in 3+1 dimensions, we develop a (lightly) categorified notion of biracks [22, 26, 27, which we call "W-bikoids" . The connection to loop-excitations in $(3+1)$-dimensional topological higher gauge theory is in $\$ 2.3$.

[1.4] A birack $(X, /, \backslash)$ is a set $X$ with two operations $X \times X \rightarrow X$ written $(x, y) \mapsto y / x$ and $(x, y) \mapsto x \backslash y$ such that $(x, y) \mapsto(y / x, x \backslash y)$ defines an invertible map $S: X \times X \rightarrow X \times X$, satisfying the set-theoretical Yang-Baxter equation [28, $(S \times$ id $) \circ($ id $\times S) \circ(S \times$ id $)=($ id $\times S) \circ(S \times$ id $) \circ($ id $\times S)$; and for any $a \in X$ the maps $x \mapsto x / a$ and $x \mapsto x \backslash a$ are invertible. Our conventions for biracks are spelled out in $\$ 4.3$,

As we now recall, a birack encodes the combinatorics of virtual braid diagrams (and more generally of virtual knot and link diagrams) [18, 19, 20, 29, 30, up to Reidemeister II and III moves between them.

[1.5] Given a birack $(X, /, \backslash)$, a $(X, /, \backslash)$-colouring [26, 31] of a virtual braid diagram (more generally of a virtual link diagram [17, 18, 19, 20]) is a map from the set of edges of the diagram to $X$, satisfying the relations below when four edges meet at a positive, negative or virtual crossing:
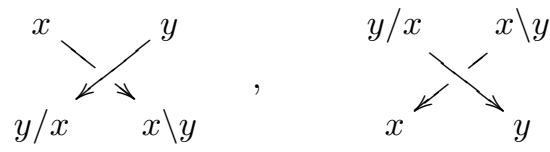

and

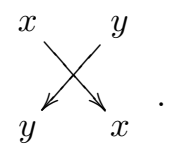


(A crossing breaks an edge into two components, regardless of it being over, under or virtual crossing.)

A biquandle is a birack also imposing Reidemeister I moves [26, 31] between virtual knot diagrams. The axioms of biquandles ensure that the number of $(X, /, \backslash)$-colourings of a virtual link diagram is invariant under all classical Reidemeister moves. This implies that the number of $(X, /, \backslash)$-colourings is also invariant under virtual and mixed Reidemeister moves between virtual link diagrams [20], hence defining a virtual link invariant, called the colour counting invariant [31.

A welded birack 22] (called here a $W$-birack) makes the number of $(X, /, \backslash)$-colourings of virtual braid diagrams in addition invariant under the welded move (6). In the biquandle case this leads to the definition of invariants of welded knots [17, 18, 20, 32, 33, 34.

[1.6] A birack $\left(X, /, \backslash\right.$ ) yields, for each $n$, a (in this paper right) action $\triangleleft$ of $\operatorname{VB}_{n}$ on $X^{n}$. The action on $\underline{x}=\left(x_{1}, x_{2}, \ldots, x_{n}\right) \in X^{n}$ of $S_{a}^{+}[n]$ and of $V_{a}[n]$ is given by the bottom lines of the diagrams below:

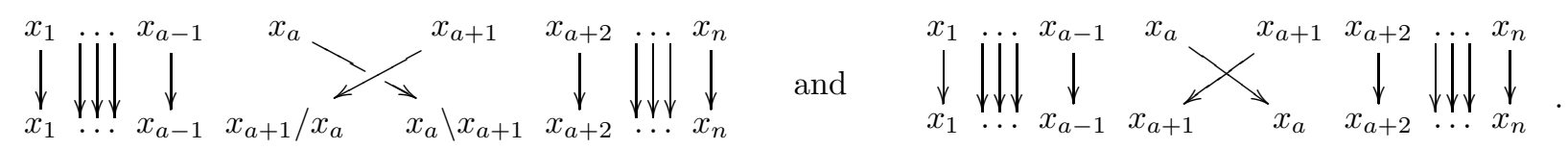

This is to say that:

$$
\begin{aligned}
\left(x_{1}, \ldots, x_{a-1}, x_{a}, x_{a+1}, \ldots, x_{n}\right) \triangleleft S_{a}^{+}[n] & =\left(x_{1}, \ldots, x_{a-1}, x_{a+1} / x_{a}, x_{a} \backslash x_{a+1}, x_{a+2}, \ldots, x_{n}\right), \\
\left(x_{1}, \ldots, x_{a-1}, x_{a}, x_{a+1}, \ldots, x_{n}\right) \triangleleft V_{a}[n] & =\left(x_{1}, \ldots, x_{a-1}, x_{a+1}, x_{a}, x_{a+2}, \ldots, x_{n}\right) .
\end{aligned}
$$

Linearising, we have a right-representation of $\mathrm{VB}_{n}$ on $(\mathbb{C} X)^{n \otimes} \cong \mathbb{C}\left(X^{n}\right)$.

There are several ways to enrich biquandle colouring counting invariants of links and welded links. See for instance [35, 36, 37, for quandle cohomology classes, and [38, 39] for meridian-longitude refinements. In this paper we give the first representation theoretic steps in the development of a light categorification-based enrichment. We will focus on the corresponding representations of the loop braid group. Invariants of welded and virtual knots will be addressed elsewhere [40].

The underlying notion is that of a bikoid $\left(\Gamma, X_{\Gamma}^{+}\right)$; see 4.4 . Let us sketch their definition. First of all, given a groupoid [41] $\Gamma$, we write $\Gamma_{0}$ for the set of objects, $\Gamma_{1}$ for the set of morphisms (arrows), and $\sigma, \tau: \Gamma_{1} \rightarrow \Gamma_{0}$ for the source and target maps. We write $\Gamma^{n}$ for the $n$-fold product groupoid and $\Gamma^{2 n}$ for the wreath product 84.1 the semidirect product $\Gamma^{n} \rtimes \Sigma_{n}$ with the symmetric group $\Sigma_{n}$ acting by permutation of factors. We draw elements of $\Gamma^{2 n}$ as top-to-bottom oriented permutation diagrams (like $V_{a}[n]$ in (4) above) with edges decorated by elements of $\Gamma_{1}$. Multiplication is then by vertical stacking, followed by the composition of the groupoid arrows living in the same strand of a permutation diagram.

A bikoid is a groupoid $\Gamma$ and a birack $\left(\Gamma_{0}, /, \backslash\right)$, called the underlying birack of the bikoid, together with two maps $\Gamma_{0} \times \Gamma_{0} \rightarrow \Gamma_{1}$, of the form:

$$
(x, y) \mapsto(x \stackrel{L(x, y)}{\longrightarrow} x \backslash y) \text { and }(x, y) \mapsto(y \stackrel{R(x, y)}{\longrightarrow} y / x) .
$$

The $L, R$ maps are combined as $L \otimes R$ in $\Gamma^{22}$, and $X_{\Gamma}^{+}=(L \otimes R) T$, where $T$ is the elementary transposition. A bikoid can be graphically represented as in Equation (8) below. The over-under effect in the last version of $X_{\Gamma}^{+}(x, y)$ carries no information. But this redundant information is useful for later tracking reasons, and in order to formulate bikoid colourings of braid diagrams, in a parallel way to birack colourings as in (7).

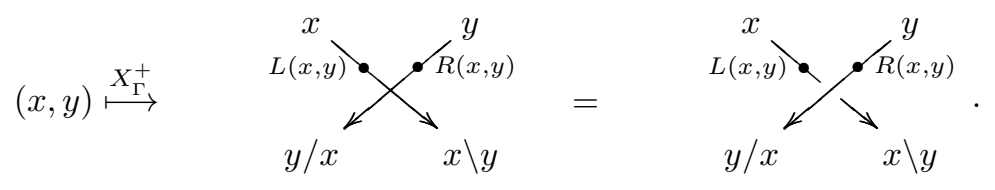

The axioms that $X_{\Gamma}^{+}$in Equation (8) should obey are in Def. 22, A graphical way to state them is in Equation (61), which, 'component-wise' (i.e. only looking at the $L, R$ maps) is equivalent to Equation (62).

As we will see, constructions for bikoids appear in finite (2-)group topological gauge theory. There, the $L(x, y)$ and $R(x, y)$ arrows encode Aharonov-Bohm phases [3, 42, 43, 44, 45] arising from flat connection 
holonomy and flat 2-connection 2-holonomy obtained when point-particles move in 2-dimensional space and loop-particles move in 3-dimensional space; see $\$ 2.1$ and $\$ 2.3$. Hence, we call $L$ and $R$ "holonomy arrows".

One can formulate the notion of a bikoid colouring of a virtual braid diagram (and more generally of a virtual link diagram), which close to a positive crossing should follow the pattern indicated in (8). (At negative crossings we have the inverse of $X_{\Gamma}^{+}$, and at virtual crossings only identity holonomy arrows are inserted.) The axioms of bikoids precisely ensure that, fixing colours of top and bottom strands, and given equivalent virtual braid diagrams $B$ and $B^{\prime}$, there is a one-to-one correspondence between colourings of $B$ and colourings of $B^{\prime}$, which moreover preserves the composition of holonomy arrows living in each strand.

[1.7] A welded bikoid (abbrv. W-bikoid) is a bikoid that also obeys the welded relation (6). In concrete terms this means (63) and (64). This ensures that the one-to-one correspondence between bikoid colourings of the paragraph above also holds if $B$ and $B^{\prime}$ are related by moves between welded braid diagrams.

[1.8] Given a bikoid $\left(\Gamma, X_{\Gamma}^{+}\right)$, then not only is $\Gamma_{0}$ a birack, but also $\Gamma_{1}$ is a birack. Thus, by [1.6] in particular we have a right-action of $\mathrm{VB}_{n}$ on $\Gamma_{1}^{n}$, hence a representation of $\mathrm{VB}_{n}$ on $\mathbb{C} \Gamma_{1}^{n}$. Let us write $\underline{\gamma} \in \Gamma_{1}^{n}$ as:

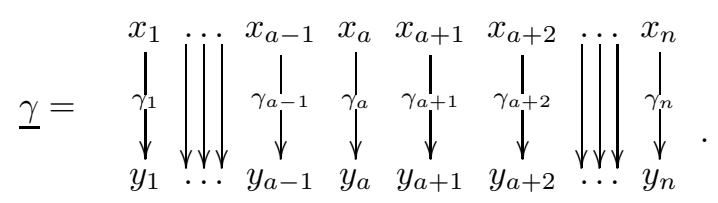

Let $\star$ denote composition of arrows in $\Gamma$. Then this representation is given by:

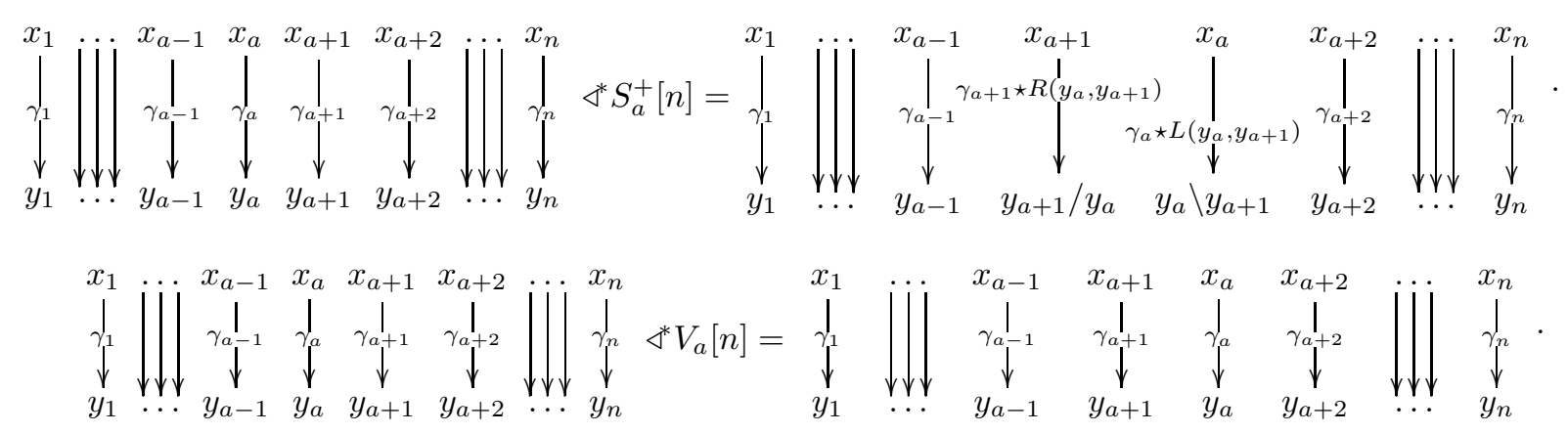

W-bikoids yield representations of the welded braid group $\mathrm{WB}_{n}$ defined in the same way.

Bikoids have a level of structure which biracks do not have, which is their underlying groupoid $\Gamma$. This leads the primary reason to introduce them. In particular consider the following.

[1.9] The groupoid algebra $\mathbb{C}(\Gamma)$ of a groupoid $\Gamma$ [46, 47, 48] is the free vector space $\mathbb{C} \Gamma_{1}$ with product:

$$
(x \stackrel{\gamma}{\rightarrow} y)\left(x^{\prime} \stackrel{\gamma^{\prime}}{\rightarrow} y^{\prime}\right)=\delta\left(y, x^{\prime}\right)\left(x \stackrel{\gamma \star \gamma^{\prime}}{\longrightarrow} y^{\prime}\right) .
$$

(Given $x, y \in \Gamma_{0}$, we put $\delta(y, x)$ to be 1 if $y=x$ and 0 otherwise.) If the set of objects of $\Gamma$ is finite, then $\mathbb{C}(\Gamma)$ is a unital algebra with unit: $1_{\mathbb{C}(\Gamma)}=\sum_{x \in \Gamma_{0}} \iota(x)=\sum_{x \in \Gamma_{0}}\left(x \stackrel{\mathrm{id}_{x}}{\longrightarrow} x\right)$. We also have a ${ }^{*}$-structure [3.8]

A bikoid $\left(\Gamma, X_{\Gamma}^{+}\right)$gives rise to the following invertible element in $\mathbb{C}(\Gamma) \otimes \mathbb{C}(\Gamma)$ :

$$
\mathcal{R}_{\left(\Gamma, X_{\Gamma}^{+}\right)}=\mathcal{R}=\sum_{x, y \in \Gamma_{0}}(x \stackrel{L(x, y)}{\longrightarrow} x \backslash y) \otimes(y \stackrel{R(x, y)}{\longrightarrow} y / x) \in \mathbb{C}(\Gamma) \otimes \mathbb{C}(\Gamma) .
$$

This $\mathcal{R}$ satisfies the relation below (cf. the relation satisfied by an R-matrix in a quasi-triangular bialgebra):

$$
\mathcal{R}_{12} \mathcal{R}_{13} \mathcal{R}_{23}=\mathcal{R}_{23} \mathcal{R}_{13} \mathcal{R}_{12}, \text { in } \mathbb{C}(\Gamma) \otimes \mathbb{C}(\Gamma) \otimes \mathbb{C}(\Gamma) ;
$$

see e.g. [24, Thm. VIII.2.4]. Here

$$
\mathcal{R}_{13}=\sum_{x, y \in \Gamma_{0}}(x \stackrel{L(x, y)}{\longrightarrow} x \backslash y) \otimes \operatorname{id}_{\mathbb{C}(\Gamma)} \otimes(y \stackrel{R(x, y)}{\longrightarrow} y / x), \quad \mathcal{R}_{12}=\mathcal{R} \otimes \operatorname{id}_{\mathbb{C}(\Gamma)} \text { and } \mathcal{R}_{23}=\operatorname{id}_{\mathbb{C}(\Gamma)} \otimes \mathcal{R} .
$$


(N.B.: $\mathcal{R}$ is not in general an R-matrix [24], and $\mathbb{C}(\Gamma)$ is not, a priori, a quasi-triangular bialgebra.) Furthermore, a bikoid is welded if, and only if, in $\mathbb{C}(\Gamma) \otimes \mathbb{C}(\Gamma) \otimes \mathbb{C}(\Gamma)$, it holds that:

$$
\mathcal{R}_{13} \mathcal{R}_{23}=\mathcal{R}_{23} \mathcal{R}_{13}
$$

A main result of this paper is that the representation in [1.8] of $\mathrm{VB}_{n}$ can be generalised to braid any $n$-tuple $\left(V_{1}, \ldots, V_{n}\right)$ of representations of $\mathbb{C}(\Gamma)$ - see Thm. 38 and 41 In particular, (11) implies that, if $V$ is a representation of $\mathbb{C}(\Gamma)$, then there is a representation $\triangleleft^{*}$ of $\mathrm{VB}_{n}$ on $V^{n \otimes}=V \otimes \ldots \otimes V$, such that:

$$
\begin{gathered}
\left(v_{1} \otimes \ldots \otimes v_{a-1} \otimes v_{a} \otimes v_{a+1} \otimes v_{a+2} \ldots \otimes v_{n}\right) \triangleleft^{*} S_{a}^{+}(n) \\
=\sum_{x, y \in \Gamma_{0}} v_{1} \otimes \ldots \otimes v_{a-1} \otimes v_{a+1} \cdot(y \stackrel{R(x, y)}{\longrightarrow} y / x) \otimes v_{a} \cdot(x \stackrel{L(x, y)}{\longrightarrow} x \backslash y) \otimes v_{a+2} \otimes \ldots \otimes v_{n}, \\
\left(v_{1} \otimes \ldots \otimes v_{a-1} \otimes v_{a} \otimes v_{a+1} \otimes v_{a+2} \ldots \otimes v_{n}\right) \triangleleft^{*} V_{a}(n)=v_{1} \otimes \ldots \otimes v_{a-1} \otimes v_{a+1} \otimes v_{a} \otimes v_{a+2} \otimes \ldots \otimes v_{n} .
\end{gathered}
$$

This is a unitary representation if $V$ is a unitary representation of the groupoid algebra. This coincides with the representation in 1.8] if $V=\mathbb{C}(\Gamma)$. If $\left(\Gamma, X_{\Gamma}^{+}\right)$is a welded bikoid, then this representation of $\mathrm{VB}_{n}$ in $V^{n \otimes}$ descends to a representation of $\mathrm{WB}_{n}$. In particular the welded move (6) follows from Equation (12).

If $\mathbb{C}(\Gamma)$ can be given a quasi-triangular bialgebra structure and $\mathcal{R}$ in (10) is its R-matrix then (13) yields exactly the braid group representation yielded by the quasi-triangular structure in $\mathbb{C}(\Gamma)$; see [24, VIII-3].

Of course the utility of all this abstract machinery depends on the ability to construct bikoids and W-bikoids. We address this core point now.

Let $G$ be a finite group. An example of unitary representation of the welded braid group that follows from W-bikoids is the representation of $\mathrm{WB}_{n}$ on $V^{n \otimes}$, arising from the R-matrix in the quantum double $D(G) \cong \mathbb{C}(\operatorname{AUT}(G))$ of the group algebra of $G[49$, which is a quasi-triangular Hopf algebra. Here $V$ is a representation of $D(G)$. This represention of $\mathrm{WB}_{n}$ is normally only stated to be a representation of the braid group $\mathrm{B}_{n}$ 24, 49, 50, 51, by using (13). However (14) extends it trivially to a representation of $\mathrm{VB}_{n}$. Since the R-matrix of $D(G)$ satisfies (12), the representation in (13]14) descends to $\mathrm{WB}_{n}$.

[1.10] Let us now hence relate W-bikoids with the quantum-double $D(G)$. For $G$ a finite group, $\operatorname{AUT}(G)$ is defined to be the action groupoid of the conjugation action of $G$ on $G$. The objects of $\operatorname{AUT}(G)$ are elements $g \in G$ and arrows have the form $\left(g \stackrel{a}{\rightarrow} a g a^{-1}\right)$, where $g, a \in G$. Composition in $\operatorname{AUT}(G)$ is by group multiplication in reverse order. Hence in $\mathbb{C}(\operatorname{AUT}(G))$ the product on generators is:

$$
\left(g \stackrel{a}{\rightarrow} a g a^{-1}\right)\left(g^{\prime} \stackrel{a^{\prime}}{\longrightarrow} a^{\prime} g^{\prime} a^{\prime-1}\right)=\delta\left(a g a^{-1}, g^{\prime}\right)\left(g \stackrel{a a^{\prime}}{\longrightarrow} a a^{\prime} g a^{\prime-1} a^{-1}\right), \text { where } g, g^{\prime}, a, a^{\prime} \in G .
$$

[1.11] The quantum double $D(G)$ is the algebra $\mathbb{C}(\operatorname{AUT}(G))$ made 24] a quasi-triangular Hopf algebra by:

$$
\begin{array}{ll}
\Delta\left(x \stackrel{g}{\rightarrow} g x g^{-1}\right)=\sum_{y z=x}\left(y \stackrel{g}{\rightarrow} g y g^{-1}\right) \otimes\left(z \stackrel{g}{\rightarrow} g z g^{-1}\right), & \epsilon\left(\left(x \stackrel{g}{\rightarrow} g x g^{-1}\right)\right)=\delta\left(x, 1_{G}\right), \\
S\left(x \stackrel{g}{\rightarrow} g x g^{-1}\right)=\left(g x^{-1} g^{-1} \stackrel{g^{-1}}{\longrightarrow} x^{-1}\right), & \mathcal{R}=\sum_{g, h \in G}\left(g \stackrel{h^{-1}}{\longrightarrow} h^{-1} g h\right) \otimes\left(h \stackrel{1_{G}}{\longrightarrow} h\right) .
\end{array}
$$

[1.12] The bikoid $X_{G}^{+}$associated to $\operatorname{AUT}(G)$ is given, cf. Equation (8), by:

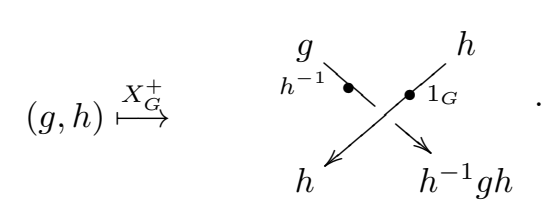

Therefore $\mathcal{R}_{\left(\Gamma, X_{G}^{+}\right)}$obtained from Equation (10) coincides with $\mathcal{R}$ in Equation (16). Note that the underlying birack of $X_{G}^{+}$is the 'conjugation quandle' in $G: h / g=h$ and $g \backslash h=h^{-1} g h$; see [35, 36, 37].

So we can see that even though $X_{G}^{+}$in (17) is apparently only a very simple spin-off of the conjugation quandle, the holonomy arrows in $X_{G}^{+}$add the information needed for generating the representations of the braid group derived from the R-matrix in the quantum double $D(G)$. Hence $X_{G}^{+}$is considerably stronger 
than the conjugation quandle of $G$ alone. In particular, the invariants of knots derived from the $R$-matrix of $D(G)$ take into account not only the knot group of a knot, as does the conjugation quandle of $G$, but also [40] the entire peripheral structure of a knot [38], which the conjugation quandle alone cannot uncover.

As we will see below in $\$ 2.1$, the holonomy arrows in $X_{G}^{+}$naturally arise from Aharonov-Bohm phases expected in finite group topological field theory in the 2-disk; see [42, 43, 44, 45, 52, 53].

[1.13] For future reference, for $X_{G}^{+}$, the representation of $\operatorname{VB}_{2}$ on $\mathbb{C}(\operatorname{AUT}(G)) \otimes \mathbb{C}(\operatorname{AUT}(G))$ from [1.8] is:

$$
\begin{aligned}
& \left(\left(a^{-1} g a \stackrel{a}{\rightarrow} g\right) \otimes\left(b^{-1} h b \stackrel{b}{\rightarrow} h\right)\right) \triangleleft^{*} S_{1}^{+}[2]=\left(b^{-1} h b \stackrel{b}{\rightarrow} h\right) \otimes\left(a^{-1} g a \stackrel{h^{-1} a}{\rightarrow} h^{-1} g h\right), \\
& \left(\left(a^{-1} g a \stackrel{a}{\rightarrow} g\right) \otimes\left(b^{-1} h b \stackrel{b}{\rightarrow} h\right)\right) \triangleleft^{*} V_{1}[2]=\left(b^{-1} h b \stackrel{b}{\rightarrow} h\right) \otimes\left(a^{-1} g a \stackrel{a}{\rightarrow} g\right) .
\end{aligned}
$$

[1.14] Our main example here (39) and (97) is a W-bikoid $X_{R}^{+}$in a certain groupoid $\operatorname{TRANS}\left(T_{R}^{2}(\mathcal{G})\right)$ motivated by discrete higher gauge theory in the 3 -disk. Here $\mathcal{G}$ is a finite 2 -group (represented by a crossed module). In this case, the underlying birack of $X_{R}^{+}$is a full fledged W-birack and not simply a quandle.

In this $\left(\operatorname{TRANS}\left(T_{R}^{2}(\mathcal{G})\right), X_{R}^{+}\right)$case, no underpinning quasi-triangular Hopf algebra appears to be in hand for modelling the representations of the welded braid group thereby obtained. Groupoid algebras are examples of weak Hopf algebras [54, 55, 56. However the representations of the welded braid group derived from $\operatorname{TRANS}\left(T_{R}^{2}(\mathcal{G})\right)$ do not appear to arise from a bona fide R-matrix inside $\mathbb{C}\left(\operatorname{TRANS}\left(T_{R}^{2}(\mathcal{G})\right)\right.$ ), at least as far as the definition of quasi-triangular weak Hopf algebras appearing in [55] is concerned. These difficulties in interpreting our representations of $\mathrm{WB}_{n}$ in terms of already know construction were our main reason to introduce bikoids, which in addition have the already mentioned advantage that they naturally incorporate Aharonov-Bohm phases featuring in topological field theory in their very construction.

[1.15] The W-bikoid $X_{R}^{+}$, motivated by higher gauge theory, can be derived from one of the form $X_{g r}^{+}$, below. Here we have an abelian $g r$-group, i.e. a group $G$ left-acting, by automorphisms, in an additive abelian group $A$. Form the semidirect product $G \ltimes A$ and define $\operatorname{TRANS}(G, A)=\operatorname{AUT}(G \ltimes A)$ to be the action groupoid of the conjugation action of $G \ltimes A$ on itself. Thus arrows of $\operatorname{TRANS}(G, A)$ are of the form:

$$
\left.\left((g, a) \stackrel{(w, k)}{\longrightarrow}(w, k)(g, a)(w, k)^{-1}\right)=\left(w g w^{-1}, k+w \triangleright a-\left(w g w^{-1}\right) \triangleright a\right)\right), \text { where } g, w \in G \text { and } a, k \in A .
$$

The corresponding W-bikoid $X_{g r}^{+}$takes the form:

$$
X_{g r}^{+}((z, a),(w, b))=\underbrace{(z, a)}_{\left(w, a+b-w^{-1} \triangleright a\right)}\left(\begin{array}{c}
\left(w^{-1}, 0_{A}\right) \\
\left(w_{G}^{-1} z w, w^{-1} \triangleright w^{-1} \triangleright a\right)
\end{array}\right.
$$

This W-bikoid is very different from the $X_{G \ltimes A}^{+}$we would obtain from (17).

A topological explanation for the existence of the underlying W-birack of $X_{g r}^{+}$, in terms of elementary algebraic topology - namely in terms of the action of $\pi_{1}$ on $\pi_{2}$, is done in 4.5 .1 . (The pertinent space is the 3-disk $D^{3}$ minus an unlinked union of unknotted circles.) The latter W-birack had appeared previously in [32, where it arised from Yetter TQFT [57, 58, of the complement of a knotted surface in $S^{4}$ [59, 60]. In [32] it is proven that this W-birack can distinguish between different welded knots with the same knot group.

In a future publication [40, we will inspect invariants of virtual and welded links derived from bikoids.

\section{Structure of the paper}

In 92 , we sketch the reason of why W-bikoids appear in the context of topological gauge theory in $D^{2}$ and topological higher gauge theory in $D^{3}$. We start in $\$ 2.1$ by giving an overview of Bais Flux metamorphosis 42, 43, 44, 45, and how it is related to quantum doubles and finite group W-bikoids. In $\$ 2.2$ we give a brief overview of discrete higher gauge theory, and in particular of the 2-dimensional holonomy of a 2-connection and its behaviour under gauge transformations. In 2.3 we explain how our main example of W-bikoids is related to loop-particles moving in $D^{3}$. In $\$ 2.3$ we also propose a version of Bais flux metamorphosis in order to handle higher gauge fluxes of unknotted and unlinked loop particles in topological higher gauge theory. Section 2 is roughly independent of the rest of the paper. However it motivates the main constructions. 
In $₫ 3$ we recap conventions for: groupoids, groupoid algebras, virtual and welded braid groups, and loop braid groups, and sketch the definition of the isomorphism $\mathrm{WB}_{n} \rightarrow \mathrm{LB}_{n}$. In 4 we firstly 4.1 explain the wreath product $\Gamma^{2 n}$ of a groupoid with the symmetric group $\Sigma_{n}$, and in 4.2 we give a graphical calculus for $\Gamma^{2 n}$, providing a visual framework to handle a lot of intricate calculations. Conventions for biracks are in 4.3. Bikoids and W-bikoids are defined in 4.4 In 4.5 we explain the W-bikoids $X_{g r}^{+}$derived from abelian $g r$-groups, and show how they arise from elementary algebraic topology. In $\$ 5$ we show how W-bikoids give rise to representations of the loop braid group, when loops are coloured with representations of the groupoid algebra of the underlying groupoid of the W-bikoid. In $\$ 6$ we show how finite 2-groups (described as crossed modules) yield W-bikoids, hence proving that the representations of $\mathrm{LB}_{n}$ announced in 2.3 exist.

Acknowledgements. Alex Bullivant and Paul Martin thank EPSRC for funding under Grant EP/I038683/1. João Faria Martins and Paul Martin thank the Leverhulme trust for funding under the Research Project Grant RPG-2018-029. We all thank Zoltan Kadar, Marcos Calçada and Celeste Damiani for discussions.

\section{Insights from topological gauge theory and higher gauge theory}

\subsection{Finite group gauge theory in $D^{2}$, quantum doubles and braid groups}

This section contains a review of Bais flux metamorphosis [42, 43, 44, 45, 52, 53, organised and reinterpreted with our lift to higher gauge theory in mind. (A connection between finite group topological gauge theory in $D^{2}$ and topological phases in (2+1)-dimensions can be made via the Kitaev quantum double model [16, 61.)

Let $G$ be a finite group. We consider a topological gauge theory with gauge group $G$, with spatial manifold the unit disk $D^{2}=[0,1]^{2}=\{(x, y) \mid 0 \leq x, y \leq 1\}$. Here a gauge field is interpreted as being a principal $G$-bundle. We suppose the restriction to the boundary $S^{1}$ to be 'static' in time. Note that a principal $G$-bundle, where $G$ is finite has a unique connection, which is automatically flat.

[2.1] Consider a set of $n$ anyonic point-particles $p_{1}, \ldots, p_{n}$ moving in the interior of $D^{2}$. We model these by a flat $G$-connection in $D^{2}$, which becomes singular at the location of each anyon. At that location a point-like magnetic vortex arises. The magnetic vortex at $p_{i}$ is formally classified by its magnetic flux. The latter is the gauge holonomy $g_{i} \in G$ observed when travelling along a positively oriented small circle $c_{i}$, looping around the particle $p_{i}$. We let $*_{c_{i}}$ be the coinciding initial and end-point of $c_{i}$; cf. Fig. 1. Both $c_{i}$ and $*_{c_{i}}$ will be co-moving with the particle $p_{i}$. A naive 1-particle Hilbert space is given by the group algebra $\mathbb{C} G$.

[2.2] Holonomies $g \in G$ are not physical since the holonomy around a particle is defined only up to conjugation. The reason is twofold. Firstly, gauge transformations in the connection change holonomy along a closed path by conjugation by an element of $G$. Secondly, it is more realistic to consider the connection holonomy along of a loop $\hat{c}_{i}$, looping around $p_{i}$, however starting and ending at a base-point $*$ far away from $p_{i}$. Different choices of $\hat{c}_{i}$ lead also to conjugate values for the holonomy. (In anticipation, we note that in higher gauge theory, discussed in 2.3 , the lifts of these two types of tranformation in the way we calculate magnetic flux do not act exactly in the same way on the observed 2-dimensional holonomy; see [2.23]

Hence one could consider the Hilbert space $\mathbb{C} C C(G)$ spanned by the conjugacy classes of $G$ to be the relevant one for describing finite group topological gauge theory particles in the plane. However in general there exists a further decomposition of the physical Hilbert space corresponding to internal charge degrees of freedom. These charge degrees of freedom are truncated when switching from $\mathbb{C} G$ to $\mathbb{C} C C(G)$; 62 , Page 1196]. For instance, given a flux $g \in G$, conjugation by the $g$-central subgroup $Z(g)=\{h \in G \mid g h=h g\} \subset$ $G$ is a symmetry of the flux degree of freedom and as such the action can be further decomposed into representations of $Z(g)$. The latter correspond to possibly non-trivial charges associated to anyons.

[2.3] Finally, cf. [43, 44, a convenient formulation of the Hilbert space associated to a point-particle in finite group topological gauge theory in $D^{2}$ is given by the underlying vector space of the groupoid algebra $\mathbb{C}(\operatorname{AUT}(G))$; see [1.10] This $\mathbb{C}(\operatorname{AUT}(G))$ retains enough information to allow the treatment of multiple anyons and of charge. The Hilbert space for $n$-particles configurations is then $\mathbb{C}(\operatorname{AUT}(G)) \otimes \cdots \otimes \mathbb{C}(\operatorname{AUT}(G))$.

Cf. the discussion in [62, §2.1], the vector space $\mathbb{C}(\operatorname{AUT}(G))$ is a 'resolved' version of $\mathbb{C} C C(G)$, i.e. a derived quotient of $\mathbb{C} G$ under the conjugation action. Hence switching from $\mathbb{C} C C(G)$ to $\mathbb{C}(\mathrm{AUT}(G))$ can be motivated by the general principle that derived quotients retain more information and are as a rule well behaved in comparison to naive quotients, see e.g. [63, §2.1]. The latter point of view will be prevalent when discussing topological higher gauge theory in $D^{3}$; see $\$ 2.3$. 


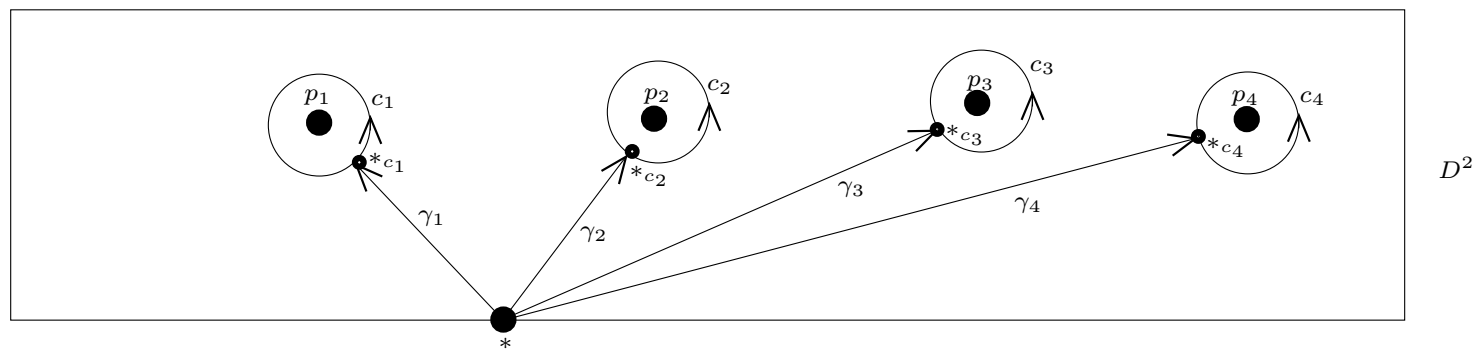

Figure 1: A generic configuration of four particles $p_{1}, p_{2}, p_{3}, p_{4}$ in the disk $D^{2}$.

[2.4] In general, if $X$ is a set of possible formal configurations of a particle and $H$ is a group of symmetries acting on $X$, then we retain enough information in the Hilbert space of the particle if we take this space to have basis the morphisms of the action groupoid (Def. 2) of the action of $H$ on $X$. A 'resolved' state of the system is thus a formal state $x \in X$ together with a datum $h \in H$, which encodes how it was measured.

An inner product in $\mathbb{C}(\operatorname{AUT}(G))$ which renders different arrows orthogonal is:

$$
\left\langle\left(g \stackrel{a}{\rightarrow} a g a^{-1}\right),\left(g^{\prime} \stackrel{a^{\prime}}{\rightarrow} a^{\prime} g^{\prime} a^{\prime-1}\right)\right\rangle=\delta\left(a, a^{\prime}\right) \delta\left(g, g^{\prime}\right) .
$$

Note that the group $G$ left-acts in $\mathbb{C}(\operatorname{AUT}(G))$ by $\langle$,$\rangle -unitary transformations as:$

$$
h .\left(g \stackrel{a}{\rightarrow} a g a^{-1}\right)=\left(g \stackrel{h a}{\longrightarrow} h a g a^{-1} h^{-1}\right), \text { where } g, h, a \in G .
$$

The groupoid algebra $\mathbb{C}(\operatorname{AUT}(G))$ is additionally an example of a Hopf algebra (see [1.11] describing the local symmetries of finite group topological gauge theory [43, 44, 45, 61] in the 2-disk. References justifying the latter fact by identifying the quantum-double algebra as the coarse graining algebra for topological gauge theory are [53, 64. Particle types in finite-group topological gauge theory should thus be labelled by irreducible representation of $\mathbb{C}(\operatorname{AUT}(G))$.

A Hilbert space on its own has very little information (Hilbert spaces of equal dimension are isomorphic). We must specify what the observables are and what they mean physically.

Given a $g \in G$, the flux operator $F_{g}: \mathbb{C}(\operatorname{AUT}(G)) \rightarrow \mathbb{C}(\operatorname{AUT}(G))$ for a single particle is defined as $F_{g}\left(x \stackrel{a}{\rightarrow} a x a^{-1}\right)=\delta\left(g, a x a^{-1}\right)\left(x \stackrel{a}{\rightarrow} a x a^{-1}\right)$. Classically, particle fluxes depend on the way we travel around a particle and also on the choice of gauge. Let us specify what the flux observables mean by stating how the magnetic flux (i.e. the connection holonomy around each particle) is calculated.

[2.5] Let our base-point $*$ be $(1 / 2,0) \in \partial D^{2}$. For simplicity, we will only consider gauge transformations on our discrete gauge fields which are the identity in $\partial D^{2}$. This is a very strong restriction, which we will also make when discussing topological higher gauge theory in 2.3, see [2.18] Given that our gauge fields are stable in $*$, we can also choose and fix a point in the fibre of $*$. Hence the holonomy along a path starting and ending at $*$ will from now on be uniquely defined. This will not make the definition of flux around a particle $p_{i}$ unique, as some freedom still exists in the actual choice of closed path looping around $p_{i}$.

Let $c_{i}$ be a counter-clockwise oriented path enclosing $p_{i}$ and no other $p_{j}$ for $j \neq i$, with initial point $*_{c_{i}}$, lying on the straight line connecting the base-point $* \in \partial D^{2}$ and $p_{i}$. We then choose a path $\gamma_{i}$, call it a "connecting path", connecting the base-point $*$ to the initial point $*_{c_{i}}$. This leads to an unambiguous definition of magnetic flux $g_{i} \in G$ of a discrete gauge field around $p_{i}$, to be the holonomy along the closed path $\hat{c}_{i}=\gamma_{i} c_{i} \gamma_{i}^{-1}$, connecting $*$ to $*$. Homotopically distinct choices of paths in the definition of $\gamma_{i}$ generally yield conjugate values for $g_{i}$. However in the following, by allowing the motion of the particles $p_{1}, \ldots, p_{n}$, there is no way paths $\gamma_{i}, i=1, \ldots, n$ connecting $*$ to $*_{c_{i}}$ can be chosen both once and for all, and in a way such that they depend continuously of time. For this reason we will consider particles in generic positions.

[2.6] Let us say that the particles $p_{1}, \ldots, p_{n}$ are in generic positions if their $x$-coordinates are all different (see Fig. 11). In particular straight lines $\gamma_{i}$ connecting $*$ to $*_{c_{i}}$ do not intersect any particle. For generic configurations of particles, we will assume that the flux of each particle corresponds to the one arising from the holonomy along $\gamma_{i} c_{i} \gamma_{i}^{-1}$, for $\gamma_{i}$ a straight line connecting $*$ to $*_{c_{i}}$.

In the following we utilise the convention that the tensor components in the Hilbert space $\mathbb{C}(\operatorname{AUT}(G)) \otimes$ $\cdots \otimes \mathbb{C}(\operatorname{AUT}(G)$ correspond to the particles ordered by increasing $x$ value, here left to right. 
[2.7] We now consider the transformations on the Hilbert space under motions of the particles. Moving particles adiabatically in a way such that the configuration remains generic does not change the state of the system. Let the particles $p_{i}, i=1, \ldots, n$ move, in between $t=0$ and $t=1$, in such a way that we momentarily pass (say at $t=1 / 2$ ) through a non-generic configuration. For each $i$, and at each $t$, let $\gamma_{i}^{t}$ be the path, in $D^{2} \backslash\left\{p_{1}, \ldots, p_{n}\right\}$, obtained from a straight line from $*$ to the position of $*_{c_{i}}$, at time $t$. Note that $\gamma_{i}^{t}$ might be undefined at $t=1 / 2$, since the straight line from $*$ to $*_{c_{i}}$ may cross another particle.

For each $i$, let also $\phi_{i}^{t}$ be the path obtained by concatenating $\gamma_{i}^{0}$ with the trajectory of $*_{c_{i}}$ in between time 0 and time $t$. Note that $\phi_{i}^{0}=\gamma_{i}^{0}$ and that $\phi_{i}^{t}$ is homotopic to $\gamma_{i}^{t}$, if $0 \leq t<1 / 2$. However $\gamma_{i}^{t}$ is in general not homotopic to $\phi_{i}^{t}$ for $t>1 / 2$. See Fig. 2] below, for a two particles configuration.
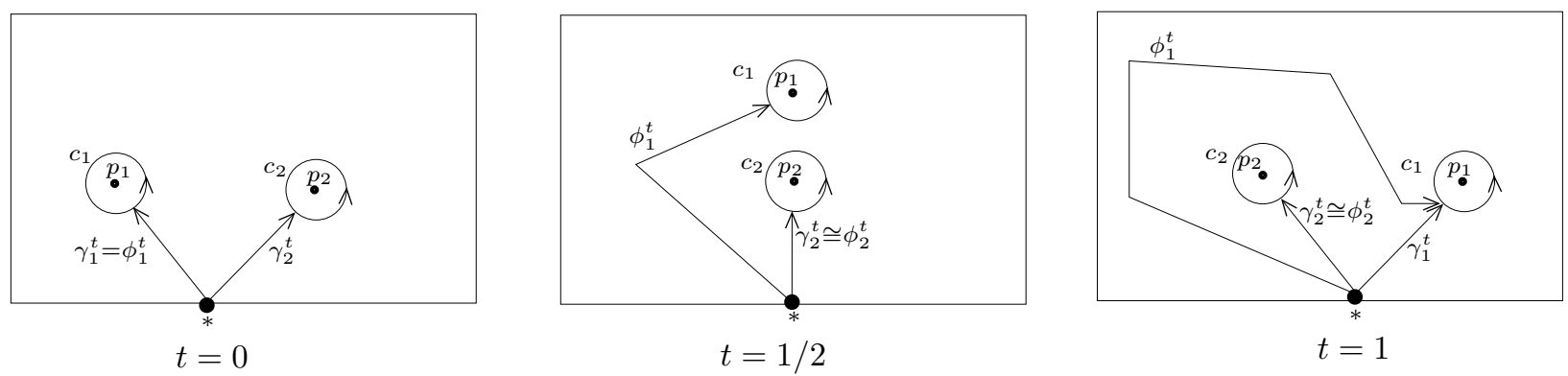

Figure 2: A movement of particles passing non-generic configurations. Note $\gamma_{2}^{t}$ is homotopic to $\phi_{2}^{t}$, for all $t$.

When passing by non-generic configurations, 'Aharonov-Bohm phases' 42, 43, 44, 45, 65, $A_{i} \in G$ must be inserted. They make up for the fact that $\gamma_{i}^{t}$ and $\phi_{i}^{t}$ might not be homotopic when $t=1$. A possible convention is that, for each $i=1, \ldots, n$, the group element $A_{i}$ is given by the holonomy of the $G$-connection along the path $\phi_{i}^{t}\left(\gamma_{i}^{t}\right)^{-1}$, at time $t=1$.

These holonomies $A_{i}$ can be determined [42, 43, 44, 45]. For instance in Fig 2, if the particle $p_{1}$ carries flux $g$, and the particle $p_{2}$ carries flux $h$, then the holonomy along the path $\phi_{2}^{t}\left(\gamma_{2}^{t}\right)^{-1}$ is trivial, when $t=1$, i.e. $A_{2}=1_{G}$. This is because the path is homotopically trivial, hence the associated holonomy is the group identity. On the other hand, the holonomy along $\phi_{1}^{t}\left(\gamma_{1}^{t}\right)^{-1}$, at $t=1$, is $A_{1}=h^{-1}$. (Since at $t=1, \phi_{1}^{t}\left(\gamma_{1}^{t}\right)^{-1}$ is homotopic to $\gamma_{2}^{t} c_{2}^{-1}\left(\gamma_{2}^{t}\right)^{-1}$.) Therefore, the movement of particles in Fig. 2 induces the following unitary transformation in the Hilbert space $\mathbb{C}(\operatorname{AUT}(G)) \otimes \mathbb{C}(\operatorname{AUT}(G))$ (Bais calls this "flux metamorphosis" [42]):

$$
\begin{aligned}
\left(a^{-1} g a \stackrel{a}{\rightarrow} g\right) \otimes\left(b^{-1} h b \stackrel{b}{\rightarrow} h\right) & \mapsto A_{2} \cdot\left(b^{-1} h b \stackrel{b}{\rightarrow} h\right) \otimes A_{1} \cdot\left(a^{-1} g a \stackrel{a}{\rightarrow} g\right) \\
& =\left(b^{-1} h b \stackrel{b}{\rightarrow} h\right) \otimes h^{-1} \cdot\left(a^{-1} g a \stackrel{a}{\rightarrow} g\right) \\
& =\left(b^{-1} h b \stackrel{b}{\rightarrow} h\right) \otimes\left(a^{-1} g a \stackrel{h^{-1} a}{\rightarrow} h^{-1} g h\right) .
\end{aligned}
$$

Note that the operative part of this can be expressed as $g \otimes h \mapsto h \otimes h^{-1} g h$.

[2.8] Remark: Equation (22) is exactly (18). Hence, the bikoid $X_{G}^{+}$in (17) is related to finite group topological gauge theory in $D^{2}$ (just forget about the 'elementary transposition' component). Considering $n$-particle configurations (22) yields representations of the braid group $\mathrm{B}_{n}$ in $\mathbb{C}(\operatorname{AUT}(G))^{n \otimes}$ [42, 43].

[2.9] Fix particle locations $p_{1}, \ldots, p_{n} \in D^{2}$. Adiabatic exchanges of particles, are modelled by diffeomorphisms $\left(D^{2},\left\{p_{1}, \ldots, p_{n}\right\}\right) \rightarrow\left(D^{2},\left\{p_{1}, \ldots, p_{n}\right\}\right)$ (by definition these are diffeomorphisms $D^{2} \rightarrow D^{2}$ which are the identity in the boundary $S^{1}$ of $D^{2}$ and send $\left\{p_{1}, \ldots, p_{n}\right\}$ to $\left.\left\{p_{1}, \ldots, p_{n}\right\}\right)$. The braid group $\mathrm{B}_{n}$ is isomorphic to the group of isotopy classes of diffeomorphisms $\left(D^{2},\left\{p_{1}, \ldots, p_{n}\right\}\right) \rightarrow\left(D^{2},\left\{p_{1}, \ldots, p_{n}\right\}\right)$; see 66. 67]. In topological gauge theory, maps induced by adiabatic exchanges of particles on Hilbert spaces should depend only on isotopy classes of diffeomorphisms $\left(D^{2},\left\{p_{1}, \ldots, p_{n}\right\}\right) \rightarrow\left(D^{2},\left\{p_{1}, \ldots, p_{n}\right\}\right)$. This is the case when maps on Hilbert spaces are, as outlined above, induced by holonomies (to encode AharonovBohm phases) of flat connections in $D^{2}$ with magnetic vortices at particle locations. The same principle can be made to work in the loop braid group case and higher gauge theory in the 3-disk $D^{3}$, as we now explain.

\subsection{Higher gauge theory with a finite 2-group: preliminaries}

Higher gauge theory is a generalisation of gauge theory which enables the definition of non-abelian holonomies 
along surfaces embedded in a manifold $M$, when a principal 2-bundle with a 2-connection over $M$ is given; see [11, 12, 68, 69, 70. See [70] for a cubical approach [71 to the holonomy of a 2-connection. This is the point of view used here. In particular, a 2-bundle with a 2-connection over $M$, will be subordinated to an open cover of $M$, whose open sets are called coordinate neighbourhoods [70, §3.1].

In discrete higher gauge theory, for the gauge field, instead of a principal bundle with a finite group $G$ of structure, we have a principal 2-bundle (see [12, 72] and [70, §3.1]) with a structure finite 2-group. Note that a principal 2-bundle with a finite 2-group of structure has a unique 2-connection.

[2.10] In the strict version of higher gauge theory used here, a 2-group is equivalent to a crossed module $\mathcal{G}=(\partial: E \rightarrow G, \triangleright)$ of groups, [71, 73, 74]. Here $G$ and $E$ are groups, $\partial: E \rightarrow G$ is a group map, and $\triangleright$ is a left action of $G$ on $E$ by automorphisms, such that the 1st and 2nd Peiffer relations are satisfied: $\partial(g \triangleright e)=g \partial(e) g^{-1}$ and $\partial(e) \triangleright f=e f e^{-1}$, where $g \in G$ and $e, f \in E$. We will suppose that $E$ and $G$ are finite.

A square in $\mathcal{G}$ [70, $\$ 2.2 .1]$ is, by definition, a diagram like:

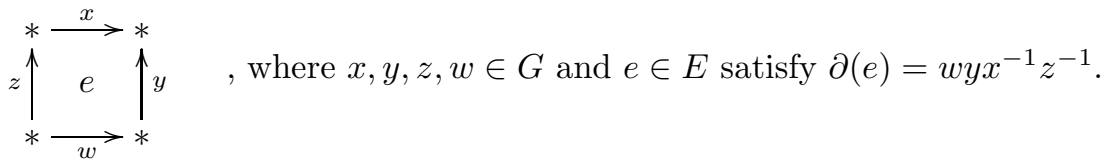

[2.11] Squares in $\mathcal{G}$ can be composed vertically and horizontally, if their sides match [70, §2.2.1]:

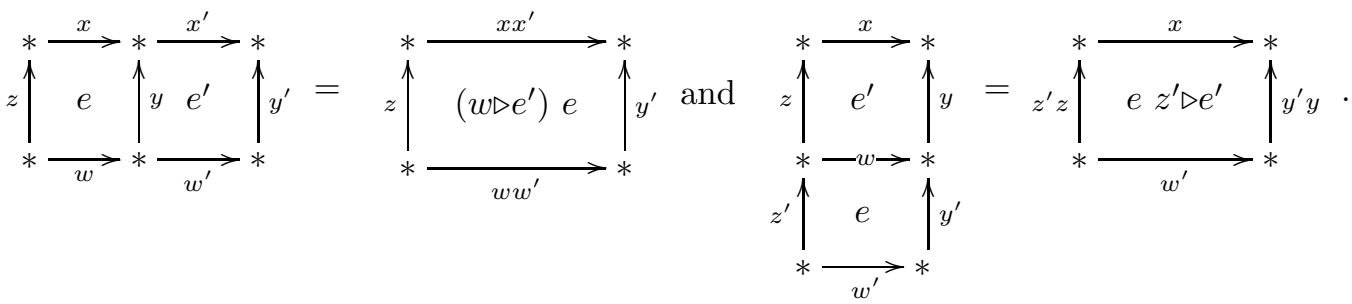

These compositions are associative, have vertical and horizontal identities, and satisfy the interchange law; see [70, 2.2.1], 71, §6.6] or [8] for explanation. Also, each square in $\mathcal{G}$ has a vertical and a horizontal inverse.

In higher gauge theory, fields are 2-connections in a 2-bundle over a manifold $M$. A 2-connection is a cubical- $\mathcal{G}$-2-bundle with a connection, as defined in [70, Defs. 3.1 and 3.4]. There is an equivalence relation [70. Def. 4.18] on the set of 2-connections, by gauge transformations. It is derived from a 2-groupoid of 2connections, gauge transformations and 2-gauge transformations between gauge transformations [68, 69, 70].

[2.12] If we have a 2 -connection in $M$, then given a piecewise smooth map $P:[0,1] \times[0,1] \rightarrow M$, and a choice of coordinate neighbourhoods covering the image $P\left([0,1]^{2}\right)$, we can define [70, Def. 5.1] the 2-dimensional holonomy $2 \operatorname{hol}(P)$ along $P$, which is a square in $\mathcal{G}[8,70,71$, as in (23). This $2 \mathrm{hol}(P)$ depends only on the choice of coordinate neighbourhoods covering $P\left(\partial[0,1]^{2}\right)$; see [70, Cor. 5.5].

If $\gamma:[0,1] \rightarrow M$ is a path, there is also a 1-dimensional holonomy $\operatorname{hol}(\gamma) \in G$ of $\gamma$; see [70, §5.1.3]. Holonomy $h=\operatorname{hol}(\gamma)$ along a closed path is well defined, up to transformations like $h \mapsto \partial(a) g h g^{-1}$, where $g \in G$ and $a \in E$. The elements of $G$ appearing in the edges of the square $2 \mathrm{hol}(P)$ in (23) are given by the 1-dimensional holonomies of the paths obtained by restricting $P$ to the edges of the boundary of $[0,1]^{2}$.

[2.13] Piecewise smooth maps of form $P:[0,1] \times[0,1] \rightarrow M$ can be composed horizontally, respectively vertically, when the restrictions to the appropriate side of $[0,1] \times[0,1]$ match, by rescaling [70, $§ 2.3 .1]$.

The 2-dimensional holonomy of a 2-connection preserves both horizontal and vertical compositions of maps $P:[0,1]^{2} \rightarrow M$; see [70, Thm. 4.3]. Each map $\Gamma:[0,1]^{2} \rightarrow M$ has horizontal $\Gamma^{-H}$ and vertical $\Gamma^{-V}$ inverses, given by $(t, s) \mapsto \Gamma(1-t, s)$ and $(t, s) \mapsto \Gamma(t, 1-s)$. The 2-dimensional holonomy of a 2-connection preserves horizontal and vertical inverses.

Maps $P:[0,1] \times[0,1] \rightarrow M$ such that the paths $[0,1] \rightarrow M$ obtained by restricting $P$ to $\{0\} \times[0,1]$ and to $\{1\} \times[0,1]$ coincide are called tubes in $M$, as they can be seen as maps $P: S^{1} \times[0,1] \rightarrow M$. If $P$ is a tube 
in $M$, the 2-dimensional holonomy along $P$ has the form:

$$
\operatorname{2hol}(P)=\stackrel{*}{h \uparrow_{* \underset{w}{\longrightarrow}}^{\stackrel{x}{\longrightarrow}} *} \uparrow_{h}^{*} \text {, where } x, h, w \in G \text { and } f \in E \text { satisfy } \partial(f)=w h x^{-1} h^{-1} \text {. }
$$

[2.14] We will also consider tori-maps in $M$, which by definition are tubes in $M$ such that the paths $[0,1] \rightarrow M$ obtained by restricting to $[0,1] \times\{0\}$ and to $[0,1] \times\{1\}$ coincide. If $P$ is a torus-map in $M$, then the 2-dimensional holonomy of a 2-connection along $P$ has the form below (cf. [70, Def. 5.16]):

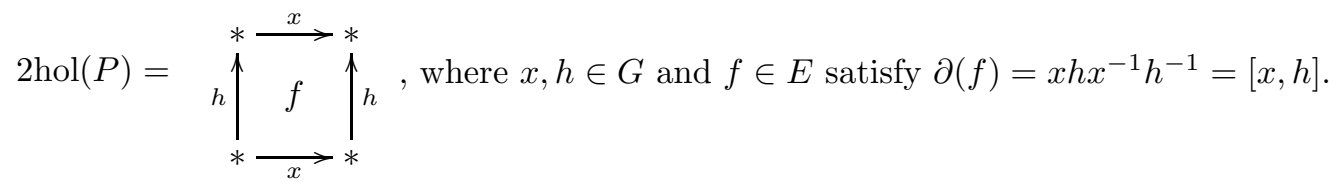

This 2 hol $(P)$, for $P$ a torus-map, is independent of the choice of coordinate neighbourhoods along $P\left(\partial[0,1]^{2}\right)$, and the gauge equivalence class of the 2-connection, up to [70, Thm. 5.17] transformations of the form of a simultaneous horizontal and vertical conjugation in the 'double-groupoid' of squares in $\mathcal{G}$, as in (27), below. (We note that the squares in the middle left and the middle right are horizontal inverses of each other, and similarly the squares in the middle top and the middle bottom are vertical inverses of each other.)

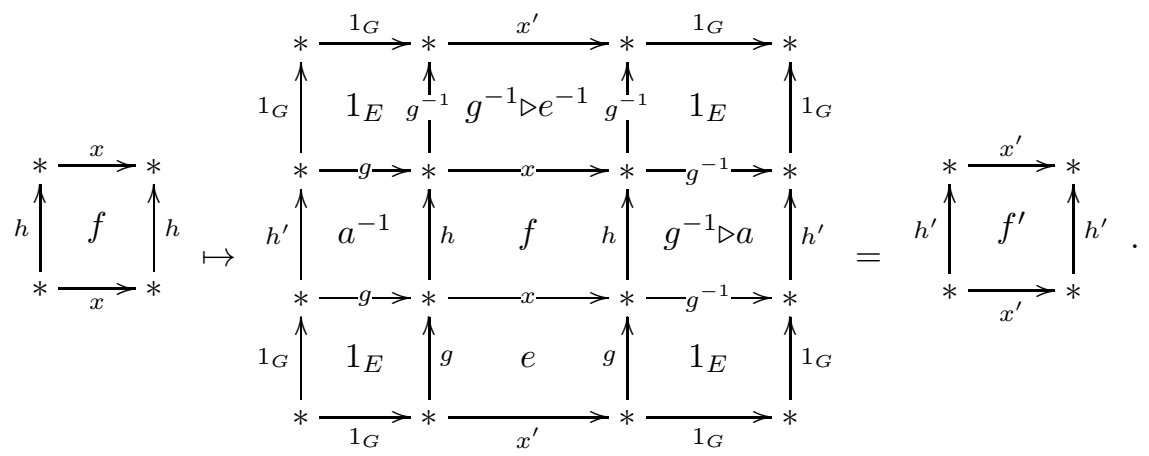

Where: $\partial(e)=x^{\prime} g x^{-1} g^{-1}$, thus $x^{\prime}=\partial(e) g x g^{-1}, \quad \partial(a)^{-1}=g h g^{-1} h^{-1}$, thus $h^{\prime}=\partial(a) g h g^{-1}$, and:

$$
f^{\prime}=e\left(g x g^{-1}\right) \triangleright a g \triangleright f\left(g h g^{-1}\right) \triangleright e^{-1} a^{-1}=x^{\prime} \triangleright a \text { e } g \triangleright f\left(g h g^{-1}\right) \triangleright e^{-1} a^{-1} .
$$

[2.15] A 2-connection is 2-flat if its categorical curvature vanishes [70, Def. 2.16] in all coordinate neighbourhoods of $M$ [68, Prop. 3.3.6]. 2-connections on 2-bundles with finite 2-groups are automatically 2-flat.

[2.16] Fix a flat 2-connection in $M$. The 2-dimensional holonomy along $P:[0,1]^{2} \rightarrow M$ depends only on the homotopy class of the map $P:[0,1]^{2} \rightarrow M$, relative to the boundary of $[0,1]^{2}$, and the choice of coordinate neighbourhoods covering $P\left(\partial[0,1]^{2}\right)$. This follows by [70, Thm. 4.5], by the same argument as in the proof of [70, Thm. 5.8], or by [68, Prop. 3.3.6]. For torus-maps $P:[0,1]^{2} \rightarrow M$, we consider homotopies of $P$ relative to $\{0,1\} \times I$, which stay within the set of torus maps. If we fix coordinate neighbourhoods covering $P(\{0,1\} \times[0,1])$, then $2 \mathrm{hol}(P)$ is homotopy invariant, up to transformations as in (27), with $e=1_{E}, g=1_{G}$.

[2.17] Let $B^{2}=\left\{z \in \mathbb{R}^{2}:\|z\| \leq 1\right\}$ be based at $*=(-1,0)$. Hence $B^{2}$ and $D^{2}$ are homeomorphic. Suppose that $P: B^{2} \rightarrow M$ is piecewise smooth. It is more convenient to consider the 2-dimensional holonomy along $P$ to have the form: $2 \operatorname{hol}(P)=* e v$, where $e \in E$ and $v=\partial(e)$; cf. (23), here we are putting $v=w y x^{-1} z^{-1}$. Note that $v$ is the 1-dimensional holonomy of the path obtained by restricting $P$ to the boundary $S^{1}$ of $B^{2}$, oriented counterclockwise. If $B \subset M$ is diffeomorphic to $B^{2}$, and has a base point $* \in \partial B$, then the 2-dimensional holonomy along an orientation and base-point preserving diffeomorphism $B^{2} \rightarrow B$ depends only on $B$, see [8, Thm. 67$]$ or [70, Thm. 5.14]. It will be denoted $2 \mathrm{hol}(B)$. 


\subsection{Higher gauge theory with a finite 2-group and invariants of loop braids}

Consider a 2-flat 2-connection [2.15] in the 3-disk $D^{3}=\{(x, y, z) \mid 0 \leq x, y, z \leq 1\}$. Let us also assume that the 2-connection is 'static' in the boundary $\partial D^{3}$. We choose an oriented 'base-loop' $O \cong S^{1}$ embedded in $D^{2} \times\{1\} \subset \partial D^{3}$. We let $* \in O$ be the base point of $O$. Hence $O$ is the image of a path $\gamma:[0,1] \rightarrow \partial D^{3}$, starting and ending at $*$. Also $O$ bounds a 2-dimensional ball $B$ contained in $\partial D^{3}$; see Fig. 3 , below.

[2.18] A parameter we will need is the 2-dimensional holonomy [2.17] along $B$ which has the form:

$$
2 \mathrm{hol}(B)=\hat{*} \partial(R)
$$

Here $R \in E$. The 1-dimensional holonomy along $O$, with initial point $*$, is $\partial(R) \in G$. Cf. 2.5] as for gauge theory in $D^{2}$, we will only consider gauge transformations [70, §4.2.1] on 2-connections which are trivial in $\partial\left(D^{3}\right)$. We also fix coordinate neighbourhoods along $O$. Hence we take $R$ and $\partial R$ to be constant in time.

[2.19] Consider a set of small unknotted unlinked circles $c_{1}, \ldots, c_{n}$ (call them "loop particles") in $D^{3}$. Outside these, the 2-connection is flat. We allow for a 2-connection to be singular in the loop-particles $c_{1}, \ldots, c_{n}$, which therefore may carry 'higher gauge magnetic vortices'.

[2.20] We suppose that loop-particles can move, but remain horizontal. In other words each loop-particle is at each time $t$ contained in a plane (whose height may vary with $t$ ) parallel to $D^{2} \times\{0\} \subset D^{3}$.

[2.21] Let $c$ be loop-particle. A formal observable for $c$ (here called magnetic primary 2-flux) is the 2dimensional holonomy $F_{c}=2 \mathrm{hol}\left(P_{c}\right)$ along a torus-map $P_{c}:[0,1]^{2} \rightarrow D^{3}$, starting and ending at the baseloop $O$, and bounding the torus $T_{c}^{2}$ obtained as the boundary of a local neighbourhood of $c$ as in Fig. 3 below. Cf. the figure in Equation (2) for a visualisation of a tube-map homotopic to $P_{c}$.

Let us explain $P_{c}$. It is constructed as the vertical composition of three tubes in $D^{3}$. The torus $T_{c}^{2} \cong$ $S^{1} \times S^{1}$ has a meridian $m$ and a longitude $l$. Both $m$ and $l$ are assumed to be co-moving with $c$. The linking number of $l$ and $c$ should be zero. We consider a 'connecting tube' $\Gamma_{c}$ connecting $O$ to $l$. Then consider the torus-map in $D^{3}$ obtained by sweeping the torus $T_{c}^{2}$, in the obvious way, from $l$ to $l$. And finally we go back to $O$ by using the vertical inverse $\Gamma_{c}^{-V}$ of $\Gamma_{c}$. (Hence $\Gamma_{c}^{-V}(t, s)=\Gamma_{c}(t, 1-s)$.)

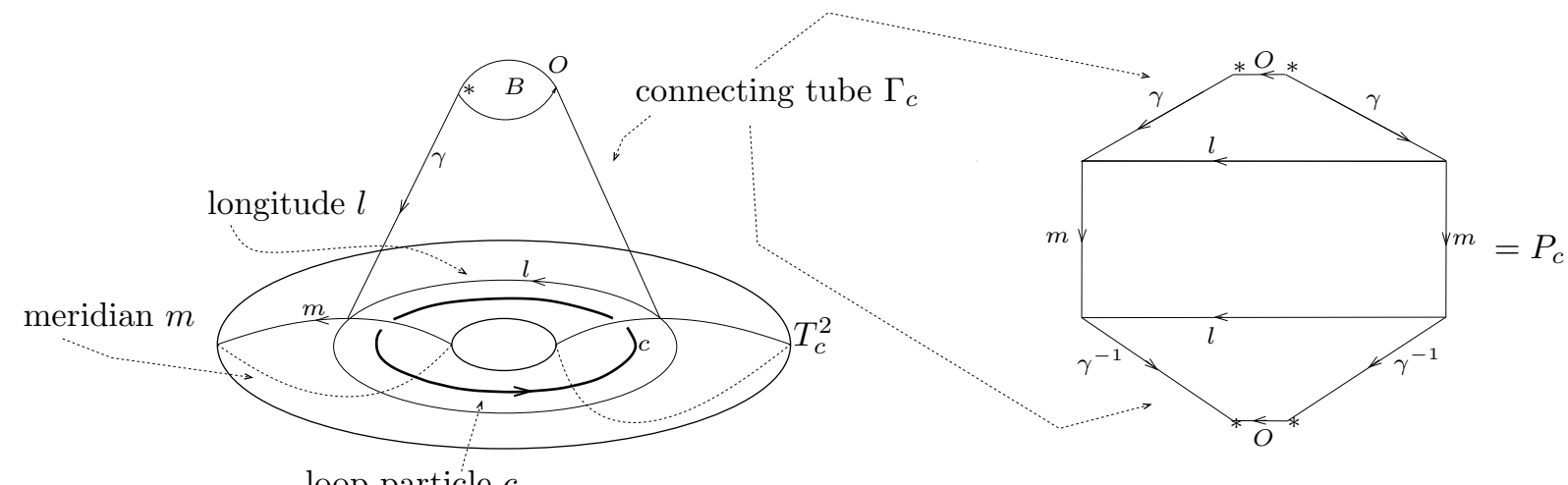

loop-particle $c$

Figure 3: Construction of a torus-map $P_{c}:[0,1]^{2} \rightarrow D^{3}$ in $D^{3}$, associated to a loop-particle $c$.

The 2-dimensional holonomy $2 \mathrm{hol}\left(P_{c}\right)$ has the form (26), and hence by [2.18] the form (29) below. Note that $h$ is the 1-dimensional holonomy along $\gamma m \gamma^{-1}$, and $\partial(R)$ is the 1-dimensional holonomy along $O$.

$$
\operatorname{2hol}\left(P_{c}\right)=\quad h \uparrow_{* \underset{\partial(R)}{*}}^{*} \uparrow_{h} \text {, where } h \in G, f \in E \text { and } \partial(f)=[\partial(R), h]
$$

[2.22] An encoding of magnetic primary 2-flux $F_{c}$ is thus a triple $F(c)=(h, \partial(R), f) \in G \times G \times E$ such that $\partial(f)=[\partial R, h]$. The relation between $F(c)=(h, \partial(R), f)$ and $2 \mathrm{hol}\left(P_{c}\right)$ is as in (29).

We let $T_{R}^{2}(\mathcal{G})$ be the set of those triples, called 2-fluxes. 
An important property of 2-fluxes is that they form a group (as do fluxes $g \in G$ of gauge theory) under the vertical composition of squares in $\mathcal{G}$; see (24). Hence: $(g, \partial(R), a)\left(g^{\prime}, \partial(R), a^{\prime}\right)=\left(g g^{\prime}, \partial(R), a g \triangleright a^{\prime}\right)$.

[2.23] As for topological gauge theory flux 2.2] $F(c)=(h, \partial(R), f)$ is not physical. It changes if we perform gauge transformations in the 2-gauge field [70, $\S 4.2 .1$ and $\S 5.1 .7$ ] or change the chosen coordinate neighbourhoods along $P_{c}\left(\partial[0,1]^{2}\right)$; see [70, Thm. 5.1.4]. Cf. 2.18] in order to simplify the construction, we fix coordinate neighbourhoods along the base loop, and the disk $B$, and only consider gauge transformations which are trivial along $\partial D^{3}$. In particular, we are considering only a subset of gauge transformations which leave the holonomy of the base-loop invariant. This means that in (27) we take $g=1_{G}$ and $e=1_{E}$. Hence $F(c)$ will change as

$$
(h, \partial(R), f) \rightarrow\left(\partial(a) h, \partial(R), R a R^{-1} f a^{-1}\right),
$$

under gauge transformations; here $e \in E$. We are using the 2nd Peiffer relation here; see [2.10],

We can also modify the connecting tube $\Gamma_{c}$, connecting the base-loop $O$ to the longitude $l$ (see Fig. 3), to a new connecting tube $\Gamma_{c}^{\prime}$. This leads to transformations in the 2-flux $F(c)=(h, \partial(R), f)$ like below:

$$
(h, \partial(R), f) \mapsto(g, \partial(R), e)(h, \partial(R), f)(g, \partial(R), e)^{-1}=\left(g h g^{-1}, \partial(R), e g \triangleright f\left(g h g^{-1}\right) \triangleright e^{-1}\right) .
$$

Here $(g, \partial(R), e) \in T_{R}^{2}(\mathcal{G})$. Note that $(g, \partial(R), e)$ arises as the 2-dimensional holonomy of the torus-map obtained as the vertical concatenation of $\Gamma_{c}^{\prime}$ and the vertical inverse of $\Gamma_{c}$.

Putting the two types of transformations together, we can see that the appropriate group of transformations on 2-fluxes is given by the following semidirect product:

$$
T_{R}^{2}(\mathcal{G}) \ltimes E=\{(g, \partial(R), e, a) \in G \times G \times E \times E: \partial(e)=[\partial(R), g]\} .
$$

with group operation

$$
(g, \partial(R), e, a)\left(g^{\prime}, \partial(R), e^{\prime}, a^{\prime}\right)=\left(g g^{\prime}, \partial(R), \text { e } g \triangleright e^{\prime}, a g \triangleright a^{\prime}\right) .
$$

The group $T_{R}^{2}(\mathcal{G}) \ltimes E$ acts on the set $T_{R}^{2}(\mathcal{G})$ of 2-fluxes as below (calculations are in $\sqrt[6.2 .2]{6}$ :

$$
(g, \partial(R), e, a) \gg(h, \partial(R), f)=\left(\partial(a) g h g^{-1}, \partial(R), R a R^{-1} \text { e } g \triangleright f\left(g h g^{-1}\right) \triangleright e^{-1} a^{-1}\right) .
$$

This action also arises from (27), where we put $x^{\prime}=\partial(R)$. (We are using the 2nd. Peiffer relation [2.10]) [2.24] We define $\operatorname{TRANS}\left(T_{R}^{2}(\mathcal{G})\right)$ as the action groupoid of the action $\gg$ of $T_{R}^{2}(\mathcal{G}) \ltimes E$ on $T_{R}^{2}(\mathcal{G})$. Objects of $\operatorname{TRANS}\left(T_{R}^{2}(\mathcal{G})\right)$ are given by 2 -fluxes $F \in T_{R}^{2}(\mathcal{G})$. Morphisms are, where $T=(g, \partial(R), e, a) \in T_{R}^{2}(\mathcal{G}) \ltimes E$ :

$$
((h, \partial(R), f) \stackrel{(g, \partial(R), e, a)}{\longrightarrow}(g, \partial(R), e, a) \gg(h, \partial(R), f))=(F \stackrel{T}{\rightarrow} T \gg F) .
$$

[2.25] In higher gauge theory, we not only have gauge transformations between 2-connections but also 2-gauge transformations between gauge transformations. Hence there is also a quotient groupoid $\overline{\operatorname{TRANS}\left(T_{R}^{2}(\mathcal{G})\right)}$ of $\operatorname{TRANS}\left(T_{R}^{2}(\mathcal{G})\right)$, where gauge transformation connected by 2-gauge transformations are identified. Throughout most of the paper, we neglect the role of 2-gauge transformations. We will come back to this in [2.37] [2.26] Finally, we note that a different type of 2-flux $\hat{F}(c) \in T_{R}^{2}(\mathcal{G})$, called thin 2-flux, can be associated to $c$ in Fig. 3. This $\hat{F}(c)$ is given by the 2-dimensional holonomy of a 'degenerate' (i.e 1-dimensional) tube $\hat{P}_{c}$, from $O$ to $O$. This $\hat{P}_{c}$ is obtained from contracting $O$ to its base point $*$, along $B$, then concatenating with a 1-dimensional tube tracing $\gamma m \gamma^{-1}$, and then using $B$ to go back to $O$ again. Cf. the figure in (3) for a visualisation of a tube-map homotopic to $\hat{P}_{c}$. We have a group morphism $\Theta_{R}: T_{R}^{2}(\mathcal{G}) \rightarrow T_{R}^{2}(\mathcal{G})$ given by $(g, \partial(R), e) \mapsto\left(g, \partial(R), R g \triangleright R^{-1}\right)$. From the assumptions in [2.18], we can conclude that primary and thin 2-fluxes of a loop-particle $c$ are related as:

$$
\hat{F}(c)=\Theta_{R}(F(c)) .
$$

Cf. 2.3] and [2.4] In analogy with $\mathbb{C}(\operatorname{AUT}(G))$ in topological gauge theory, for an unknotted loopparticle $c$, an approximation of the algebra of local symmetries of finite 2-group topological higher gauge theory in $D^{3}$ is the groupoid algebra $\mathbb{C}\left(\operatorname{TRANS}\left(T_{R}^{2}(\mathcal{G})\right)\right)$. Elementary loop-particles in our simplified model 
correspond to irreducible representations of $\mathbb{C}\left(\operatorname{TRANS}\left(T_{R}^{2}(\mathcal{G})\right)\right)$. The latter algebra is semisimple, as is any finite groupoid algebra over $\mathbb{C}$.

Now recall the $G$-action on $\operatorname{AUT}(G)$ in (21). We have a left-action "." of $T_{R}^{2}(\mathcal{G}) \ltimes E$ on $\operatorname{TRANS}\left(T_{R}^{2}(\mathcal{G})\right)$, such that:

$$
T^{\prime} .(F \stackrel{T}{\rightarrow} T \gg F)=\left(F \stackrel{T^{\prime} T}{\longrightarrow}\left(T^{\prime} T\right)>F\right),
$$

where $F \in T_{R}^{2}(\mathcal{G})$ and $T, T^{\prime} \in T_{R}^{2}(\mathcal{G}) \ltimes E$. (We more generally should have a 2-group action [75] of the underlying 2-group in $\operatorname{TRANS}\left(T_{R}^{2}(\mathcal{G})\right)$. This will be developed in a forthcoming publication.)

Let proj: $D^{2} \times[0,1] \rightarrow D^{2}$ be $(x, y, z) \mapsto(x, y)$. Consider two loop-particles $c_{1}, c_{2} \in D^{2} \times[0,1]$ in generic position. This means that $\operatorname{proj}\left(c^{1} \cup c^{2}\right)$ is the disjoint union of two circles, such that no circle is nested inside the other, and moreover the middle-points of the disks spanned by $c_{1}$ and $c_{2}$ have different $x$ coordinates.

[2.27] If $c_{1}$ and $c_{2}$ are in generic position, we can consider connecting tubes $\Gamma_{1}$ and $\Gamma_{2}$, given by rectilinear cylinders connecting the base-loop $O$ to their their longitudes $l_{1}$ and $l_{2}$, such that the line connecting the

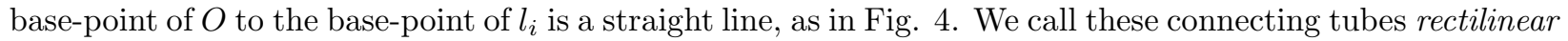
connecting tubes. Assuming that the configuration is generic, these rectilinear connecting tubes $\Gamma_{1}$ and $\Gamma_{2}$ do not intersect the regular neighbourhoods of the other loop-particle, if these regular neighbourhoods, with boundary $T_{c_{1}}^{2}$ and $T_{c_{2}}^{2}$, are chosen to be thin enough.

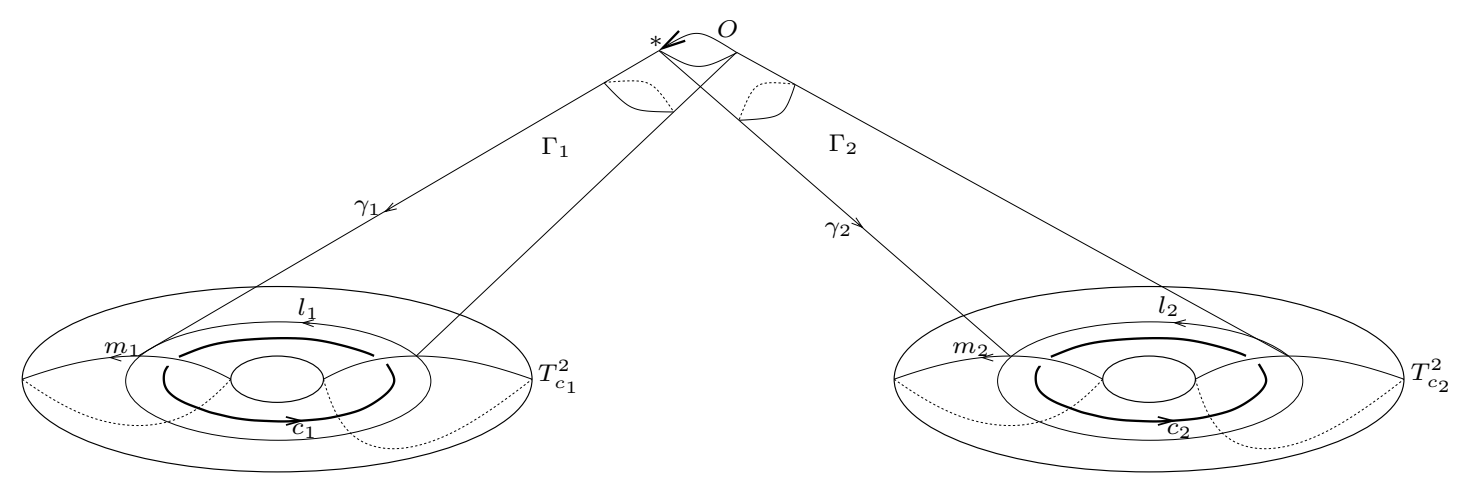

Figure 4: A generic configuration of loop-particles $c_{1}$ and $c_{2}$.

[2.28] The Hilbert space $\mathbb{C}\left(\operatorname{TRANS}\left(T_{R}^{2}(\mathcal{G})\right) \otimes \mathbb{C}\left(\operatorname{TRANS}\left(T_{R}^{2}(\mathcal{G})\right)\right.\right.$ describes the set of internal states for the pair of loop-particles, $c_{1}$ and $c_{2}$, in generic position. We order circles from left to right. The primary and thin 2-fluxes $F\left(c_{i}\right)$ and $\hat{F}\left(c_{i}\right)$ of the loop-particle $c_{i}$ are calculated by using a rectilinear tube from $O$ to $l_{i}$.

We now wish to consider the transformations on the Hilbert space arising when the loop-particles $c_{1}$ and $c_{2}$ move. Note, cf. [2.9] that if there is an Aharonov-Bohm like effect, then the pertinent mapping class group is now the loop braid group $\mathrm{LB}_{2}$ in two circles 3.4

[2.29] Fix a motion of our system, and in particular a motion of each loop $c_{i}$, and hence of each longitude $l_{i}$. Let $\Gamma_{i}^{t}$ be the rectilinear tube $\mathbf{2 . 2 7}$ from $O$ to the longitude $l_{i}$, at time $t$, where $i=1,2$. Let $\operatorname{Traj}_{i}^{\left(t_{0}, t_{1}\right)}$ be the 'tube' in $D^{3}$ made by the trajectory of the longitute $l_{i}$ between $t_{0}$ and $t_{1}$. Let $A_{i}\left(t_{0}, t_{1}\right) \in T_{R}^{2}(\mathcal{G})$ be the 2-dimensional holonomy of the torus $\mathrm{T}_{i}^{t_{0}, t_{1}}=\Gamma_{i}^{t_{0}} \operatorname{Traj}_{i}^{\left(t_{0}, t_{1}\right)}\left(\Gamma_{i}^{t_{1}}\right)^{-V}$, namely:

$$
A_{i}\left(t_{0}, t_{1}\right)=2 \operatorname{hol}\left(\Gamma_{i}^{t_{0}} \operatorname{Traj}_{i}^{\left(t_{0}, t_{1}\right)}\left(\Gamma_{i}^{t_{1}}\right)^{-V}\right),
$$

where $\left(\Gamma_{i}^{t_{1}}\right)^{-V}$ is the vertical inverse of $\Gamma_{i}^{t_{1}}$.

Our model is that the Aharonov-Bohm phase applied to $c_{i}$ for a motion from $t_{0}$ to $t_{1}$ is given by: $A_{i}=\left(A_{i}\left(t_{0}, t_{1}\right), 1_{E}\right) \in T_{R}^{2}(\mathcal{G}) \ltimes E$. Thus for example if the motion exchanges the loops we will have:

$$
\left(\stackrel{T_{1}}{\longrightarrow}\right) \otimes\left(\stackrel{T_{2}}{\longrightarrow}\right) \mapsto\left(\stackrel{A_{2} T_{2}}{\longrightarrow}\right) \otimes\left(\stackrel{A_{1} T_{1}}{\longrightarrow}\right) .
$$

[2.30] The 2-dimensional holonomy along a torus-map is not homotopy invariant as explained in 2.16] whereas in the motion group picture for loop braids groups, the trajectories Traj $\mathrm{j}_{i}^{\left(t_{0}, t_{1}\right)}$ are only defined up to 
isotopy [13]. Hence, when determining the 2-dimensional holonomy along $\mathrm{T}_{i}^{t_{0}, t_{1}}$ we must fix a representative for the homotopy class of $\mathrm{T}_{i}^{t_{0}, t_{1}}$.

Crucially for the construction in this paper, these representatives are chosen to derived from the torusmaps $P_{c}$ and $\hat{P}_{c}$ with which we calculate the primary and thin 2-flux of our loop particles $c$ [2.28] so we always look to the 'closest' $P_{c}$ and $\hat{P}_{c}$ to $\mathrm{T}_{i}^{t_{0}, t_{1}}$. Additionally we will assume that homotopically trivial tubes give rise to trivial 2-holonomies. That such strong assumptions give rise to a loop-braid group representation is a mystery to the authors which will be investigated in future work. With such approximations a notion of Aharonov-Bohm phases can be determined from the primary $F(c)$ and thin $\hat{F}(c)$ 2-fluxes of our loop particles $c$. We now explain this.

[2.31] Suppose that between $t=0$ and $t=1$ two loops $c_{1}$ and $c_{2}$ exchange places as indicated in (32), below:
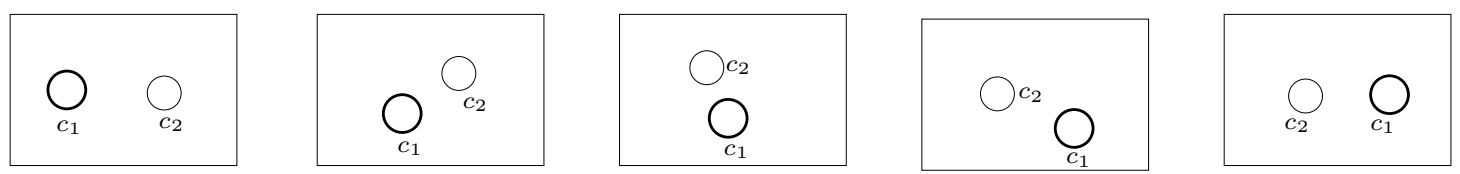

- these, and further such figures below, are views from $O$ in the ceiling of $D^{3}$. As the 2-dimensional holonomies associated to the tubes traced by $c_{1}$ and $c_{2}$ in (32) are trivial, since the homotopy classes of $\mathrm{T}_{i}^{t_{0}, t_{1}}$ are trivial, for $i=1,2$, then by [2.30] the Aharonov-Bohm phases to insert in (31) are trivial. Thus this move is associated to the following map $V$ on the Hilbert space:

$$
\left(T_{1}^{-1}>F_{1} \stackrel{T_{1}}{\longrightarrow} F_{1}\right) \otimes\left(T_{2}^{-1}>F_{2} \stackrel{T_{2}}{\longrightarrow} F_{2}\right) \stackrel{(-) \triangleleft^{*} V_{1}[2]}{\longrightarrow}\left(T_{2}^{-1}>F_{2} \stackrel{T_{2}}{\longrightarrow} F_{2}\right) \otimes\left(T_{1}^{-1}>F_{1} \stackrel{T_{1}}{\longrightarrow} F_{1}\right) .
$$

(We just swap tensor components.) We will write $V$ as $(-) \triangleleft^{*} V_{1}[2]$ in anticipation of the role of $\mathrm{LB}_{2}$.

[2.32] Now suppose that the loop-particles $c_{1}$ and $c_{2}$ swap positions in the way indicated in figure (34)-(35) below. We need to determine Aharonov-Bohm phases $A_{1}, A_{2} \in T_{R}^{2}(\mathcal{G}) \ltimes E$ associated to the movement of the loop-particles. We suppose that this movement of loop-particles happens in between $t=0$ and $t=3$.
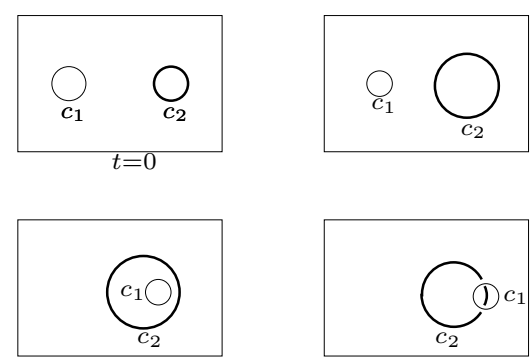
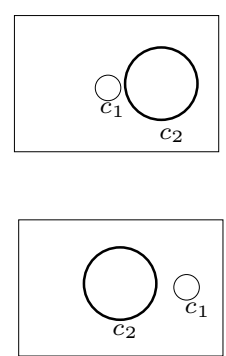
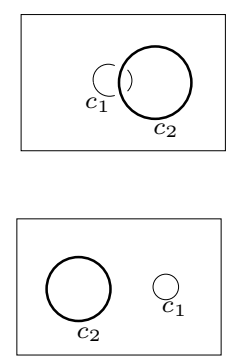
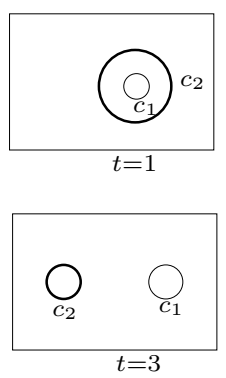

[2.33] In order to determine $A_{1}, A_{2}$, first recall [2.22] and [2.26] that a loop-particle $c_{i}$ has two 2-fluxes, primary and thin, associated to it, denoted $F\left(c_{i}\right)$ and $\hat{F}\left(c_{i}\right)$. We let $F\left(c_{i}, t\right)$ and $\hat{F}\left(c_{i}, t\right)$ be their value at time $t$. We assume that $F\left(c_{i}, t\right)$ and $\hat{F}\left(c_{i}, t\right)$ are calculated by using the rectilinear tube from $O$ to $l_{i}$. Hence $F\left(c_{i}, t\right)$ and $\hat{F}\left(c_{i}, t\right)$ are not defined for all $t$.

[2.34] We now calculate $A_{1}(0,3)=A_{1}(1,3) A_{1}(0,1)$ (recall [2.13] that the 2-dimensional holonomy of a 2connection preserves the vertical composition of tubes). Note that $A_{1}(1,3)$ is trivial (indeed $\mathrm{T}_{1}^{1,3}$ is homotopic to the constant tube at $O$ ). On the other hand, given our definition of thin and primary 2-flux of a loopparticle, we have that $A_{1}(0,1)=\hat{F}\left(c_{2}, 0\right)^{-1}$, the inverse of the thin 2-flux of $c_{2}$. Cf. [2.30] this is because $\mathrm{T}_{1}^{0,1}$ is homotopic to the inverse of the tube $\hat{P}_{c_{2}}$ in [2.26], with which we calculate the thin 2-flux of $c_{2}$. Hence, the primary 2-flux of $c_{1}$ for $t \geq 1$ is

$$
F\left(c_{1}, t\right)=\hat{F}\left(c_{2}, 0\right)^{-1} \rightarrow F\left(c_{1}, 0\right) .
$$

Here $>$ denotes the conjugation action of $T_{R}^{2}(\mathcal{G})$ on itself.

[2.35] Determining $A_{2}(0,3)$ is more complicated. Given our conventions, $A_{2}(0,1)$ is trivial. In order to calculate $A_{2}(1,3)$, we substitute (35) by an isotopic movement of loops, as in (36) below; cf. [2.30], Between $t=1$ and $t=2$, the loop-particles $c_{1}$ and $c_{2}$, exchange 'concentric position', with $c_{2}$ passing behind $c_{1}$, along the back part of the torus $T_{c_{1}}^{2}$. Hence the 2-dimensional holonomy of the trajectory of $c_{2}$ is related to the primary 2-flux of $c_{1}$ at time $t=1$. 

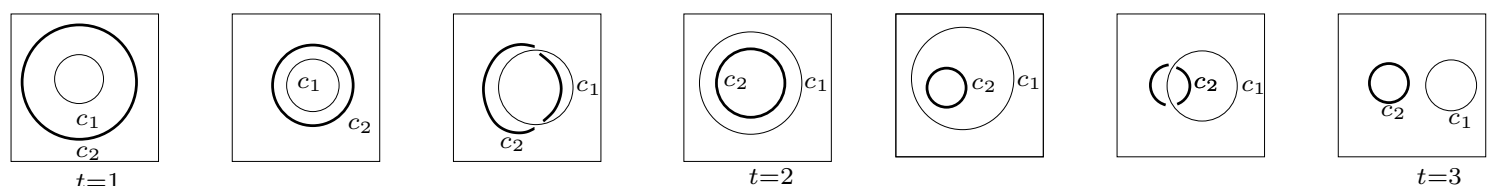

Now $A_{2}(1,3)=A_{2}(2,3) A_{2}(1,2)$. Putting together [2.29] and [2.30], we see that $A_{2}(1,2)$ is given by the inverse of the primary 2 -flux of $c_{1}$ at time 1 . This is because $\mathrm{T}_{2}^{1,2}$ is homotopic to the vertical inverse of the torus-map $P_{c_{1}}$ at time $t=2$. Hence

$$
A_{2}(1,2)=F\left(c_{1}, 1\right)^{-1} \text {. }
$$

Finally, $A_{2}(2,3)$ is given by the thin 2 -flux of $c_{1}$ at time 2 , hence $A_{2}(2,3)=\hat{F}\left(c_{1}, 2\right)=\hat{F}\left(c_{1}, 1\right)$.

Let us give explicit formulae. Recall [2.26], if

$$
F\left(c_{1}, 0\right)=(z, \partial(R), e)=F_{1} \quad \text { and } \quad F\left(c_{2}, 0\right)=(w, \partial(R), f)=F_{2},
$$

then $\hat{F}\left(c_{1}, 0\right)=\left(z, \partial(R), R z \triangleright R^{-1}\right)=\Theta_{R}\left(F_{1}\right)$ and $\hat{F}\left(c_{2}, 0\right)=\left(w, \partial(R), R w \triangleright R^{-1}\right)=\Theta_{R}\left(F_{2}\right)$. Hence:

$$
A_{1}(0,3)=\hat{F}\left(c_{2}, 0\right)^{-1}=\Theta_{R}\left(F\left(c_{2}, 0\right)\right)^{-1}=\Theta_{R}\left(F_{2}\right)^{-1} .
$$

On the other hand:

$$
\begin{aligned}
A_{2}(0,3)=A_{2}(2,3) A_{2}(1,2) & \left.=\hat{F}\left(c_{1}, 2\right)\right) F\left(c_{1}, 1\right)^{-1}=\hat{F}\left(c_{1}, 1\right) F\left(c_{1}, 1\right)^{-1} \\
& =\Theta_{R}\left(F\left(c_{1}, 1\right)\right) F\left(c_{1}, 1\right)^{-1} \\
& =\Theta_{R}\left(\hat{F}\left(c_{2}, 0\right)^{-1}>F\left(c_{1}, 0\right)\right)\left(\hat{F}\left(c_{2}, 0\right)^{-1}>F\left(c_{1}, 0\right)\right)^{-1} \\
& =\left(\Theta_{R}\left(\Theta_{R}\left(F_{2}^{-1}\right)>F_{1}^{-1}\right)\right)\left(\Theta_{R}\left(F_{2}\right)^{-1}>F_{1}\right) .
\end{aligned}
$$

[2.36] Putting the stages together, the move of loop-particles in (34) and (35) leads to the following transformation in the Hilbert space $\mathbb{C}\left(\operatorname{TRANS}\left(T_{R}^{2}(\mathcal{G})\right)\right) \otimes \mathbb{C}\left(\operatorname{TRANS}\left(T_{R}^{2}(\mathcal{G})\right)\right)$ :

$$
\left(\stackrel{T_{1}}{\longrightarrow}\right) \otimes\left(\stackrel{T_{2}}{\longrightarrow}\right) \mapsto\left(\stackrel{A_{2} T_{2}}{\longrightarrow}\right) \otimes\left(\stackrel{A_{1} T_{1}}{\longrightarrow}\right) .
$$

In full:

$$
\begin{aligned}
\left(T_{1}^{-1} \gg F_{1} \stackrel{T_{1}}{\longrightarrow} F_{1}\right) \otimes\left(T_{2}^{-1} \gg F_{2} \stackrel{T_{2}}{\longrightarrow} F_{2}\right) \stackrel{(-) \triangleleft^{*} S_{1}^{+}[2]}{\longrightarrow} A_{2} \cdot\left(T_{2}^{-1}>F_{2} \stackrel{T_{2}}{\longrightarrow} F_{2}\right) \otimes A_{1} \cdot\left(T_{1}^{-1} \gg F_{1} \stackrel{T_{1}}{\longrightarrow} F_{1}\right) \\
=\left(T_{2}^{-1}>F_{2} \stackrel{A_{2} T_{2}}{\longrightarrow} A_{2} \gg F_{2}\right) \otimes\left(T_{1}^{-1}>F_{1} \stackrel{A_{1} T_{1}}{\longrightarrow} A_{1} \gg F_{1}\right),
\end{aligned}
$$

where

$$
A_{1}=\left(\Theta_{R}\left(F_{2}\right)^{-1}, 1_{E}\right) \quad \text { and } \quad A_{2}=\left(\Theta_{R}\left(\Theta_{R}\left(F_{2}^{-1}\right)>F_{1}\right)\left(\Theta_{R}\left(F_{2}\right)^{-1}>F_{1}^{-1}\right), 1_{E}\right) .
$$

Remark 1 (Higher gauge flux metamorphosis). The unitary transformations $(-) \triangleleft^{*} V_{1}[2]$ and $(-) \triangleleft^{*} S_{1}^{+}[2]$ in $\mathbb{C}\left(\operatorname{TRANS}\left(T_{R}^{2}(\mathcal{G})\right)\right) \otimes \mathbb{C}\left(\operatorname{TRANS}\left(T_{R}^{2}(\mathcal{G})\right)\right)$, defined in Equations (33) and (38), are our proposal for a simplified higher gauge analogue of flux metamorphosis of topological gauge theory (22); see [3, 42, 43, 44, 45].

It turns out (e.g. by direct calculation) that the unitary transformations $(-) \triangleleft^{*} V_{1}[2]$ and $(-) \triangleleft^{*} S_{1}^{+}[2]$ yield a representation of the 2 -circle loop braid group $\mathrm{LB}_{2}$ in $\mathbb{C}\left(\operatorname{TRANS}\left(T_{R}^{2}(\mathcal{G})\right)\right) \otimes \mathbb{C}\left(\operatorname{TRANS}\left(T_{R}^{2}(\mathcal{G})\right)\right)$, as the physical setting would suggest. This can be extended to a representation of $\operatorname{LB}_{n}$ in $\mathbb{C}\left(\operatorname{TRANS}\left(T_{R}^{2}(\mathcal{G})\right)\right)^{n \otimes}$. One way to prove this is to note that we have an underpinning W-bikoid in $\operatorname{TRANS}\left(T_{R}^{2}(\mathcal{G})\right.$ ) of the form:

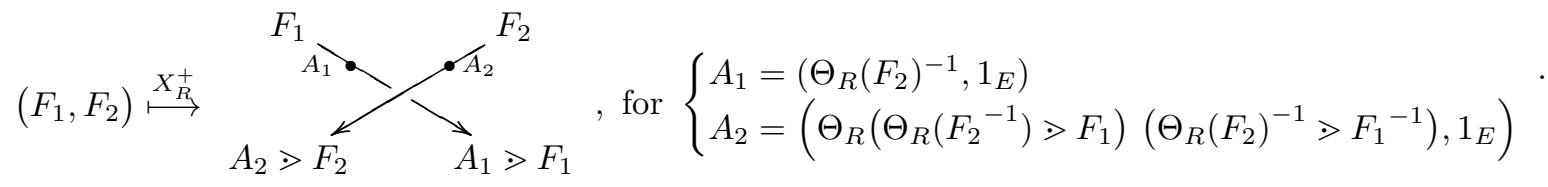


And then we can use use the general Theorems 38 and 41 . This sets the relevance of the work in this paper for modelling Aharonov-Bohm like phenomena for loop-particles in topological higher gauge theory.

It is proven in $₫ 6.2$ that all groupoids $\operatorname{TRANS}\left(T_{R}^{2}(\mathcal{G})\right)$ ), where $R \in E$, are isomorphic, and all W-bikoids $X_{R}^{+}$become the same by using a canonical groupoid isomorphism. Hence, as far as representations of $\mathrm{LB}_{n}$ are concerned, it suffices to consider the case $R=1_{E}$. In this case $T_{1_{E}}^{2}(\mathcal{G}) \cong G \rtimes_{\triangleright} A$, where $A=\operatorname{ker}(\partial) \subset E$; note that ker $\partial$ is an abelian subgroup. The arrows of $\operatorname{TRANS}\left(S^{2}(\mathcal{G})\right) \doteq \operatorname{TRANS}\left(T_{1_{E}}^{2}(\mathcal{G})\right)$ take the form:

$$
(g, a) \stackrel{(w, k, m)}{\longrightarrow}\left(\partial(m) w g w^{-1}, k+w \triangleright a-\left(w g w^{-1}\right) \triangleright k\right), \quad \text { where } g, w \in G, \quad a, k \in \operatorname{ker}(\partial) \text { and } m \in E .
$$

(Note that we switched to additive notation.) And the pertinent W-bikoid $X_{g r^{*}}^{+}$takes the form:

$$
((z, a),(w, b)) \stackrel{X_{g r_{*}^{*}}^{+}}{\longmapsto}(z, a)
$$

This $X_{g r^{*}}^{+}$is a spin-off of the abelian $g r$-group W-bikoid of (20), but the underlying groupoid of $X_{g r^{*}}^{+}$has an additional component, related to gauge transformations on 2-connections, which is however 'decoupled' from the rest of the structure. The additional level of structure present in $X_{g r^{*}}^{+}$nevertheless becomes very visible when considering 2-gauge transformations between gauge transformations (see [2.37] below), and is essential when dealing with invariants of welded knots derived from $X_{g r^{*}}^{+}$, see [40].

[2.37] For simplicity we take $R=1_{E}$. As mentioned in [2.37] there is an important variant of the groupoid $\operatorname{TRANS}\left(T_{1_{E}}^{2}(\mathcal{G})\right) \cong \operatorname{TRANS}\left(S^{2}(\mathcal{G})\right)$, where gauge transformations related by 2 -gauge transformations [69, $\S 2.3 .3]$ [70, §4.3.1] are identified. This yields a quotient groupoid $\overline{\operatorname{TRANS}\left(S^{2}(\mathcal{G})\right)}$ of $\operatorname{TRANS}\left(S^{2}(\mathcal{G})\right)$, where:

$$
\begin{aligned}
\left(( g , a ) \stackrel { ( w , k , m ) } { \longrightarrow } \left(\partial(m) w g w^{-1}, k\right.\right. & \left.\left.+w \triangleright a-\left(w g w^{-1}\right) \triangleright k\right)\right) \\
& \cong\left((g, a) \stackrel{\left(w \partial(e), k, m(w g) \triangleright e w \triangleright e^{-1}\right)}{\longrightarrow}\left(\partial(m) w g w^{-1}, k+w \triangleright a-\left(w g w^{-1}\right) \triangleright k\right)\right) .
\end{aligned}
$$

Here $g, w \in G, a, k \in \operatorname{ker}(\partial)$ and $m, e \in E$. The formulae in (41) give a bikoid structure in $\overline{\operatorname{TRANS}\left(S^{2}(\mathcal{G})\right)}$. Details on the construction of $\overline{\operatorname{TRANS}\left(S^{2}(\mathcal{G})\right)}$ and its relation to higher gauge theory will appear in [40].

\section{Preliminaries}

[3.1] If $\mathcal{C}$ is a category, the class of objects of $\mathcal{C}$ is denoted by $\operatorname{Obj}(\mathcal{C})$ or by $\mathcal{C}_{0}$. The class of morphisms of $\mathcal{C}$ is denoted by $\mathcal{C}_{1}$ or by $\operatorname{Mor}(\mathcal{C})$. If $x, y \in \mathcal{C}_{0}$, the set of morphisms $x \rightarrow y$ is denoted $\operatorname{hom}_{\mathcal{C}}(x, y)$ or $\operatorname{hom}(x, y)$.

[3.2] Our default convention for compositions in categories and groupoids is reverse to that of function composition. Given objects $x, y, z$ of $\mathcal{C}$, composition is a map $(f, g) \in \operatorname{hom}(x, y) \times \operatorname{hom}(y, z) \mapsto f \star g \in$ $\operatorname{hom}(x, z)$. However the symbol $\circ$ will always denote the usual function composition. I.e., given set maps $f: X \rightarrow Y$ and $g: Y \rightarrow Z$, then $g \circ f$ will denote the usual $(g \circ f)(x)=g(f(x))$.

[3.3] We put $\mathbb{N}=\{0,1,2, \ldots\}$ and $\mathbb{Z}^{+}=\{1,2, \ldots\}$.

[3.4] The product we use in the symmetric group $\Sigma_{n}$, of bijections $f:\{1, \ldots, n\} \rightarrow\{1, \ldots, n\}$, is $f . g=g \circ f$.

\subsection{General conventions for groupoids}

Let $\Gamma=\left(\Gamma_{1}, \Gamma_{0}, \sigma, \tau, \iota, \star\right)$ be a groupoid [76, 24, 41]. Here, $\Gamma_{1}$ is the set of morphisms (arrows) of $\Gamma, \Gamma_{0}$ is the set of objects of $\Gamma$, and $\sigma, \tau: \Gamma_{1} \rightarrow \Gamma_{0}$ denote source and target maps, respectively. Given $x, y \in \Gamma_{0}$, the set of morphisms $x \rightarrow y$ hence is $\operatorname{hom}(x, y)=\operatorname{hom}_{\Gamma}(x, y)=\left\{\gamma \in \Gamma_{1}: \sigma(\gamma)=x\right.$ and $\left.\tau(\gamma)=y\right\}$.

[3.5] An arrow $\gamma$ of $\Gamma$ will sometimes be denoted as $(x \stackrel{\gamma}{\rightarrow} y)$, thus $x=\sigma(\gamma)$ and $y=\tau(\gamma)$. 
The composition map in $\Gamma$ provides, given any triple of objects $(x, y, z) \in\left(\Gamma_{0}\right)^{3}$, a map of sets:

$$
\begin{aligned}
& \operatorname{hom}(x, y) \times \operatorname{hom}(y, z) \rightarrow \operatorname{hom}(x, z) \\
& ((x \stackrel{\gamma}{\rightarrow} y),(y \stackrel{\phi}{\rightarrow} z)) \longmapsto(x \stackrel{\gamma}{\rightarrow} y) \star(y \stackrel{\phi}{\rightarrow} z) .
\end{aligned}
$$

[3.6] The composition of arrows in $\Gamma$ will be denoted in a variety of ways, as indicated below:

$$
(x \stackrel{\gamma}{\rightarrow} y) \star(y \stackrel{\phi}{\rightarrow} z)=(x \stackrel{\gamma}{\rightarrow} y \stackrel{\phi}{\rightarrow} z)=(x \stackrel{\gamma \star \phi}{\longrightarrow} z) .
$$

[3.7] The inverse arrow to $(x \stackrel{\gamma}{\rightarrow} y)$ can, and will, be denoted in four different ways:

$$
(x \stackrel{\gamma}{\rightarrow} y)^{-1}=\left(y \stackrel{\gamma^{-1}}{\longrightarrow} x\right)=(y \stackrel{\bar{\gamma}}{\rightarrow} x)=\overline{(x \stackrel{\gamma}{\rightarrow} y)} .
$$

The identity map $\iota: \Gamma_{0} \rightarrow \Gamma_{1}$ sends $x \in \Gamma_{0}$ to the arrow $\left(x \stackrel{\mathrm{id}_{x}}{\longrightarrow} x\right)$.

Definition 2 (Action groupoid). Let the group $G$ have a left-action "." on set $X$. The action groupoid $\boldsymbol{\Gamma}_{G}(X)$ has $X$ as set of objects. The arrows have the form: $(x \stackrel{g}{\rightarrow} g \cdot x)$, where $x \in X$ and $g \in G$. The composition is such that: $(x \stackrel{g}{\rightarrow} g \cdot x) \star(g \cdot x \stackrel{h}{\rightarrow}(h g) \cdot x)=(x \stackrel{h g}{\rightarrow}(h g) \cdot x)$. (Note the order of the product.) Of course, the identity map is such that $\iota(x)=\left(x \stackrel{1_{G}}{\longrightarrow} x\right) \doteq \mathrm{id}_{x}$, where $1_{G}$ is the unit element of $G$.

Example $3(\operatorname{AUT}(G))$. Let $G$ be a group. The action groupoid of the conjugation action of $G$ on $G$ is denoted by $\operatorname{AUT}(G)$. Hence the set of objects of $\operatorname{AUT}(G)$ is $G$, and the arrows have the form $x \stackrel{g}{\rightarrow} g x g^{-1}$, where $g, x \in G$. The composition in $\operatorname{AUT}(G)$ therefore is such that:

$$
\left(x \stackrel{g}{\rightarrow} g x g^{-1}\right) \star\left(g x g^{-1} \stackrel{h}{\rightarrow} h g x g^{-1} h^{-1}\right)=\left(x \stackrel{h g}{\rightarrow} h g x g^{-1} h^{-1}\right) .
$$

\subsection{Groupoid algebras and their representations}

Definition 4 (Groupoid algebra). Let $\Gamma$ be a groupoid. Cf. [46, 47, 48]. The groupoid algebra $\mathbb{C}(\Gamma)$ of $\Gamma$ is the free $\mathbb{C}$ vector space $\mathbb{C} \Gamma_{1}$ over the set $\Gamma_{1}$ of morphisms of $\Gamma$. The product on generators is:

$$
(x \stackrel{\gamma}{\rightarrow} y) \star\left(x^{\prime} \stackrel{\gamma^{\prime}}{\rightarrow} y^{\prime}\right)=(x \stackrel{\gamma}{\rightarrow} y)\left(x^{\prime} \stackrel{\gamma^{\prime}}{\rightarrow} y^{\prime}\right)=\delta\left(y, x^{\prime}\right)\left(x \stackrel{\gamma \star \gamma^{\prime}}{\longrightarrow} y^{\prime}\right), \text { where }(x \stackrel{\gamma}{\rightarrow} y),\left(x^{\prime} \stackrel{\gamma^{\prime}}{\rightarrow} y^{\prime}\right) \in \Gamma_{1} .
$$

(Here $\delta(y, x)=1$, if $y=x$, and 0 otherwise). If $\Gamma_{0}$ is finite, then $\mathbb{C}(\Gamma)$ is a unital algebra with identity:

$$
1_{\mathbb{C}(\Gamma)}=\sum_{x \in \Gamma_{0}} \iota(x)=\sum_{x \in \Gamma_{0}}\left(x \stackrel{\mathrm{id}_{x}}{\longrightarrow} x\right) .
$$

[3.8] The groupoid algebra has a ${ }^{*}$-structure given by $(a \stackrel{\gamma}{\rightarrow} b)^{*}=\left(b \stackrel{\gamma^{-1}}{\longrightarrow} a\right)$, for all $(a \stackrel{\gamma}{\rightarrow} b) \in \Gamma_{1}$.

Remark 5. If $G$ a finite group, then $\mathbb{C}(\operatorname{AUT}(G))$ (see Example 3) is [47 the underlying algebra of the quasitriangular Hopf algebra $D(G)$ in (16), the quantum double of the group algebra of $G$; see [49, 77, 24, 50.

[3.9] In this paper a representation (or right-representation) of a unital algebra $A$ will mean a right $A$ module $V$, with action denoted $(v, a) \in V \times A \mapsto v \cdot a \in A$, such that $v \cdot 1_{A}=v$, for each $v \in V$.

Definition 6. Let $(V,\langle\rangle$,$) be an inner product space. A representation \rho:(v, k) \in V \times \mathbb{C}(\Gamma) \mapsto x . k \in V$ of $\mathbb{C}(\Gamma)$ is called unitary if it is unitary with respect to the $*$-structure in [3.8], This means that:

$$
\langle u .(x \stackrel{\gamma}{\rightarrow} y), v\rangle=\left\langle u, v \cdot\left(y \stackrel{\gamma^{-1}}{\longrightarrow} x\right)\right\rangle \text {, for all } u, v \in V \text {, and all }(x \stackrel{\gamma}{\rightarrow} y) \in \Gamma_{1} .
$$

[3.10] Suppose that $\Gamma$ is finite and $\rho$ is a finite dimensional representation of $\mathbb{C}(\Gamma)$ on $V$. Standard techniques, as e.g. in [77, page 12], prove that we can find an inner product in $V$ with respect to which $\rho$ is unitary. Standard tecniques, as in $[77$, also prove that $\mathbb{C}(\Gamma)$ is semisimple. This will be addressed elsewhere. 
Example 7. The right regular representation of $\mathbb{C}(\Gamma)$ has $\mathbb{C}(\Gamma)$ as underlying vector space. The action is by right multiplication. This representation is unitary if we pick the inner product in $\mathbb{C}(\Gamma)$ that renders different arrows orthonormal.

Example 8 (Object regular representation). The object regular representation of $\mathbb{C}(\Gamma)$ has the free vector space $\mathbb{C} \Gamma_{0}$ on $\Gamma_{0}$ as underlying vector space. The action is such that if $x \in \Gamma_{0}$ and $(a \stackrel{\gamma}{\rightarrow} b) \in \Gamma_{1}$, then:

$$
x \cdot(a \stackrel{\gamma}{\rightarrow} b)=\delta(a, x) b .
$$

This representation is unitary if we choose $\Gamma_{0}$ to be an orthonormal basis of $\mathbb{C} \Gamma_{0}$.

If $x \in \Gamma_{0}$, then $\mathbb{C} \pi_{0}(\Gamma, x)$ is a sub-representation of $\mathbb{C} \Gamma_{0}$. Here $\mathbb{C} \pi_{0}(\Gamma, x)$ is the connected component of $\Gamma_{0}$ to each $x$ belongs. As in the case of the quantum-double of a finite group algebra [77, Chapter IV], the latter representation can be modified in order to include a representation of the automorphism group of $x$.

\subsection{The virtual braid group and the welded braid group}

Virtual knot theory is discussed e.g. in [18, 20, 30. In this paper all manifolds, homeomorphisms, isotopies, immersions, embeddings, etc, are assumed to be piecewise linear [78, 79], unless otherwise specified.

Let $N$ be an oriented 1-dimensional manifold, possibly with boundary. An immersion $Q$ of $N$ in $\mathbb{R} \times[0,1]$ is the image $Q=\phi(N)$ of an immersion map $\phi: N \rightarrow \mathbb{R} \times[0,1]$; i.e. $\phi$ is piecewise linear and locally injective. The set of multiple points $M(Q)$ of $Q$ is $M(Q)=\left\{z \in Q: \# \phi^{-1}(z) \geq 2\right\}$, where \# denotes set cardinality. A double-point (also called a double intersection) is a multiple point such that $\# \phi^{-1}(z)=2$.

The interval $[0,1]$ is oriented in the positive direction. We put $p: \mathbb{R} \times[0,1] \rightarrow[0,1]$ to be $p(s, t)=t$.

Definition 9 (Virtual braid diagram). A virtual braid diagram, of degree $n$, is the image $Q$ of an immersion $\operatorname{map} \phi: \sqcup_{i=1}^{n} I_{i} \rightarrow \mathbb{R} \times[0,1]$, where $I_{i}=[0,1]$. We put $\phi_{i}: I_{i} \rightarrow \mathbb{R} \times[0,1]$ to denote the restriction of $\phi$ to $I_{i}$. The component $Q_{i} \subset Q$ of the immersion $Q$ is by definition given by $\phi\left(I_{i}\right)$, where $i=1, \ldots, n$.

We impose that: (1) multiple points of $Q$ are all transversal [80, §5.2] double intersections between different components of the immersion, (2) each double point of $Q$ is assigned a label from 'classical crossings' (positive and negative) and 'virtual crossings', as below:

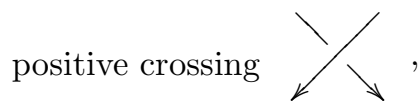

negative crossing

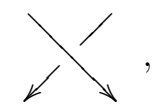

virtual crossing

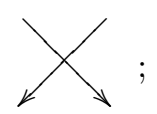

(3) for each $i \in\{1, \ldots, n\}$, the map $p \circ \phi_{i}:[0,1] \rightarrow[0,1]$ is an orientation reversion homeomorphism; (4) $Q \cap(\mathbb{R} \times\{1\})=\{1, \ldots, n\} \times\{1\}$ and $Q \cap(\mathbb{R} \times\{0\})=\{1, \ldots, n\} \times\{0\}$, (5) for each $i \in\{1, \ldots, n\}$, $\phi_{i}(1)=(i, 0) ;(6)$ the restriction of $p: \mathbb{R} \times[0,1] \rightarrow[0,1]$ to the set of double points is injective (i.e. double points occur at different heights).

We consider two virtual virtual braid diagrams $Q$ and $Q^{\prime}$ equivalent if they can be deformed one into the other via an ambient isotopy $t \mapsto f_{t}$ of $\mathbb{R} \times[0,1]$, fixing the boundary, and such that $f_{t}(Q)$ is a virtual braid diagram for each $t \in[0,1]$.

[3.11] A virtual braid diagram $Q$ yields a bijection $U_{Q}:\{1, \ldots, n\} \rightarrow\{1, \ldots, n\}$. Our convention is that $U_{Q}$ sends $j$ to the unique $i$ such that $\phi_{i}(0)=j \times\{1\}$. (Note that $\phi_{i}(1)=(i, 0)$.) See also diagram (52), below.

Definition 10 (The monoid MV $[n]$ of virtual braid diagrams). Given a positive integer $n$, we have a monoid $\operatorname{MV}[n]$ of virtual braid diagrams in degree $n$. Given $M$ and $M^{\prime}$ in $\operatorname{MV}[n]$, the multiplication $M M^{\prime}$ is the vertical juxtaposition of $M$ and $M^{\prime}$, where $M$ stays on top of $M^{\prime}$, followed by rescalling in the height direction. The identity of the monoid $\operatorname{MV}[n]$ is given by the equivalence class of the diagram $I[n]$, below:

$$
I[n]=\underbrace{\downarrow \downarrow \downarrow \downarrow \cdots \downarrow \downarrow \downarrow}_{n \text { strands }} .
$$

(Here, and also below, we put $(i, 1)=i$, where $i=1,2, \ldots, n$.) 
The monoid $\operatorname{MV}[n]$ is freely generated [18] by the equivalence classes of the diagrams displayed below:

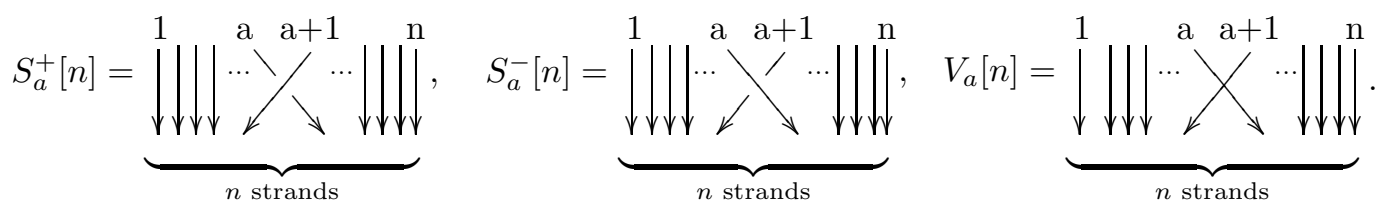

Here $a \in\{1,2, \ldots, n-1\}$. From now on, we will frequently not display some of the vertical strands which do not interact with the rest of the diagram, when drawing compositions of generators of the monoid $\operatorname{MV}[n]$. Also note that given our convention for the multiplication in $\mathrm{MV}[n]$, diagrams are read from top to bottom.

Definition 11. The $n$-strand welded braid group $\mathrm{WB}_{n}$ [18, 19, 17] is the monoid (easily proven to be a group) obtained from the monoid $\mathrm{MV}[n]$ of virtual braid diagrams, by imposing the relations (44)-(49), below. Here $a, b \in\{1, \ldots, n-1\}$ for (44) and (45), and $a \in\{1, \ldots, n-2\}$ for (46) - (49). Some relations are written algebraically and also diagramatically, since there will be several diagrammatic calculations later on.

\section{Classical and virtual Reidemeister II moves (RII ${ }^{ \pm}$and VII):}

$$
V_{a}[n] V_{a}[n] \stackrel{\mathrm{VII}}{=} I[n], \quad S_{a}^{+}[n] S_{a}^{-}[n] \stackrel{\mathrm{RII}^{+}}{=} I[n] \quad \text { and } \quad S_{a}^{-}[n] S_{a}^{+}[n] \stackrel{\mathrm{RII}^{-}}{=} I[n] .
$$

Locality, if $|a-b| \geq 2$ then:

$$
\begin{array}{lll}
V_{a}[n] V_{b}[n]=V_{b}[n] V_{a}[n], & V_{a}[n] S_{b}^{+}[n]=S_{b}^{+}[n] V_{a}[n], & V_{a}[n] S_{b}^{-}[n]=S_{b}^{-}[n] V_{a}[n], \\
S_{a}^{+}[n] S_{b}^{-}[n]=S_{b}^{-}[n] S_{a}^{+}[n], & S_{a}^{-}[n] S_{b}^{-}[n]=S_{b}^{-}[n] S_{a}^{-}[n], & S_{a}^{+}[n] S_{b}^{+}[n]=S_{b}^{+}[n] S_{a}^{+}[n] .
\end{array}
$$

Reidemeister III move (RIII): $\quad S_{a}^{+}[n] S_{(a+1)}^{+}[n] S_{a}^{+}[n] \stackrel{\text { RIII }}{=} S_{(a+1)}^{+}[n] S_{a}^{+}[n] S_{(a+1)}^{+}[n]$.

Graphically:
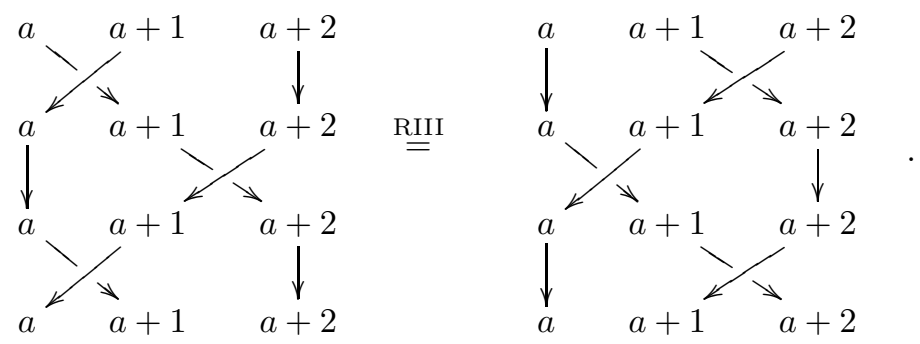

Virtual Reidemeister III move (VIII): $\quad V_{a}[n] V_{(a+1)}[n] V_{a}[n] \stackrel{\mathrm{VIII}}{=} V_{(a+1)}[n] V_{a}[n] V_{(a+1)}[n]$.

Graphically:
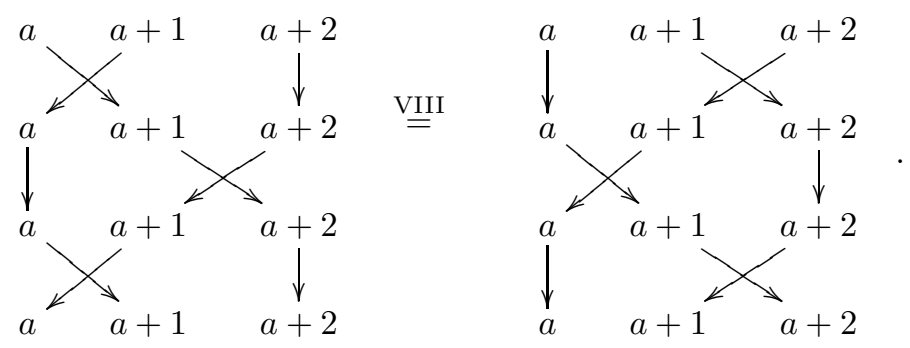

Mixed Reidemeister III move (MIII): $\quad V_{a}[n] V_{(a+1)}[n] S_{a}^{+}[n] \stackrel{\text { MIII }}{=} S_{(a+1)}^{+}[n] V_{a}[n] V_{(a+1)}[n]$.

Graphically:
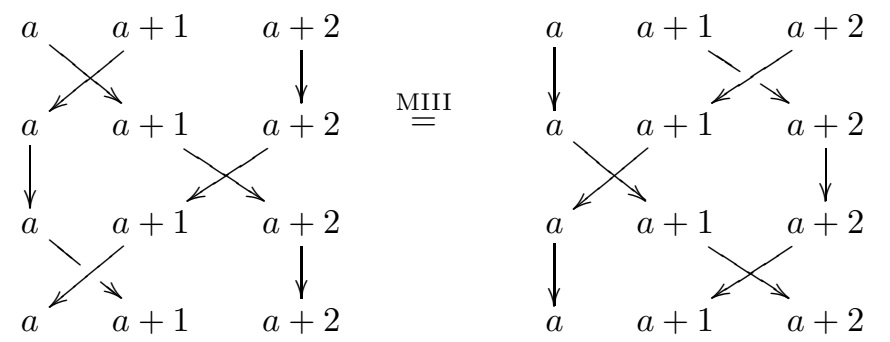
Welded Reidemeister III move (WIII): $\quad V_{a}[n] S_{(a+1)}^{+}[n] S_{a}^{+}[n] \stackrel{\mathrm{WIII}}{=} S_{(a+1)}^{+}[n] S_{a}^{+}[n] V_{(a+1)}[n]$.
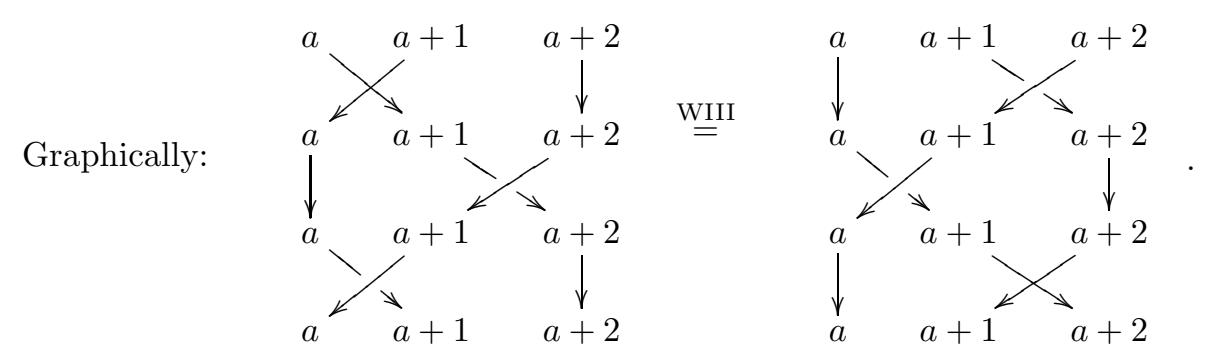

[3.12] It is easy to see that $\mathrm{WB}_{n}$ is isomorphic to the group formally generated by the symbols $S_{a}^{+}[n]$ and $V_{a}[n]$, where $a=1, \ldots, n-1$, with relations (44) to (49), desconsidering all relations involving $S_{a}^{-}[n]$, $a=1, \ldots, n-1$. This is the point of view taken in [2, 13, 14, 17. We will use the two perspectives (presentation of $\mathrm{WB}_{n}$ as a group, versus presentation of $\mathrm{WB}_{n}$ as a monoid) in this paper.

Definition 12 (Virtual braid group). The group with the same generators as $\mathrm{WB}_{n}$, and the same relations, except for the welded Reidemeister III (WIII) move (49), is called the virtual braid group $\mathrm{VB}_{n}$.

Definition 13 (Braid group). The group with generators $S_{a}^{ \pm}[n], a=1, \ldots, n-1$, and all relations in $\mathrm{WB}_{n}$ not involving the $V_{n}[a], a=1, \ldots, n-1$, is called the braid group $\mathrm{B}_{n}$ in $n$-strands.

Clearly, inclusion of generators provides an epimorphism $\mathrm{VB}_{n} \rightarrow \mathrm{WB}_{n}$.

[3.13] Consider $\Sigma_{n}$ in [3.4] with the usual presentation with generators $V_{a}[n]$, where $a=1, \ldots, n-1$, and relations: (i) $V_{a}[n] V_{(a+1)}[n] V_{a}[n]=V_{(a+1)}[n] V_{a}[n] V_{(a+1)}[n]$; (ii) $V_{a}[n] V_{b}[n]=V_{b}[n] V_{a}[n]$ if $|a-b| \geq 2$; and (iii) $V_{a}[n]^{2}=$ id. And then, concretely, $V_{a}(n)=t_{a, a+1}^{n} \in \Sigma_{n}$, the transposition exchanging $a$ and $a+1$.

[3.14] We have epimorphisms $U: \mathrm{WB}_{n}, \mathrm{VB}_{n} \rightarrow \Sigma_{n}$ sending both $S_{a}^{ \pm}[n]$ and $V_{a}[n]$ to $V_{a}[n]$. Given $B$ in $\mathrm{WB}_{n}$ or $\mathrm{VB}_{n}$, then $U(B)$, denoted $U_{B}$, is called the underlying permutation of $B$. Looking at elements of $\mathrm{WB}_{n}$ and $\mathrm{VB}_{n}$ as equivalence classes $[Q]$ of virtual braid diagrams $Q$, it holds that $U_{[Q]}=U_{Q}$ in [3.11],

Remark 14 (Tournant Dangereux). The welded Reidemeister III relation (49) is not equivalent to:

$$
S_{a}^{+}[n] S_{(a+1)}^{+}[n] V_{a}[n]=V_{(a+1)}[n] S_{a}^{+}[n] S_{(a+1)}^{+}[n] .
$$

(We have just reversed the order of the factors in (49).) The relation in (50) is equivalent to the "Forbidden move $F_{2}$ in [19]". The group obtained from $\mathrm{WB}_{n}$ by imposing (150) is called the "unrestricted virtual braid group" [19, 21. We note that virtual knot theory in the presence of the welded Reidemeister III move and its reverse (50) is trivial; see e.g. 81, 82]; however non-trivial linking phenomena may still occur [83.

Definition 15 (The monoidal categories $\mathcal{W B}$ and $\mathcal{V B}$ ). We have strict monoidal categories $\mathcal{W B}$ and $\mathcal{V} \mathcal{B}$, the welded braid category and the virtual braid category. Objects in $\mathcal{W B}$ and $\mathcal{V B}$ are given by non-negative integers. The sets of morphism $m \rightarrow m^{\prime}$ are non-empty if, and only if, $m=m^{\prime}$. Also put $\operatorname{hom}_{\mathcal{W B}}(m, m)=$ $\mathrm{WB}_{m}$ and $\operatorname{hom}_{\mathcal{V} \mathcal{B}}(m, m)=\mathrm{VB}_{m}$. On objects, the tensor product is $n \otimes n^{\prime}=n+n^{\prime}$. Given morphisms $B: n \rightarrow n$ and $B^{\prime}: n^{\prime} \rightarrow n^{\prime}$, in $\mathcal{W B}$ or $\mathcal{V} \mathcal{B}$, their tensor product $\left(B \otimes B^{\prime}\right):\left(n+n^{\prime}\right) \rightarrow\left(n+n^{\prime}\right)$ is derived from the horizontal juxtaposition $Q_{B} Q_{B}^{\prime}$ of virtual braid diagrams $Q_{B}$ and $Q_{B^{\prime}}$, for $B$ and for $B^{\prime}$, moving crossings up and down, if necessary, in order that all crossings appear at different heights.

By (45), the tensor product of morphisms is well defined and we have monoidal categories $\mathcal{W B}$ and $\mathcal{V B}$.

[3.15] The monoidal categories $\mathcal{W B}$ and $\mathcal{V B}$ can be presented by generators and relations, as in 24, §XII]. The generators are $S^{ \pm}[2]$ and $V[2]$. For $\mathcal{V B}$ the only relations are the ones in (44), for $n=2$, and (46)-(48), for $n=3$, where $S_{2}^{+}[3]=1_{1} \otimes S_{1}^{+}[2], S_{1}^{+}[3]=S_{1}^{+}[2] \otimes 1_{1}$ and so on. (We note that the locality relation (45)) is automatically satisfied in a monoidal category.) In order to present $\mathcal{W B}$, we must also add relation (49). 


\subsection{The loop braid group: isomorphism with the welded braid group}

Papers addressing the loop braid group, or close relatives, include [13, 14, 15, 67. We essentially follow [14]. In this subsection, our convention for the 3-disk is: $D^{3}=\left\{z \in \mathbb{R}^{2}:\|z\| \leq 2\right\} \times[-1,1]$. Let $n \in \mathbb{Z}^{+}$. Let $C_{n} \subset D^{3}$ be a disjoint union of unlinked circles in $D^{3} \cap\left(\mathbb{R}^{2} \times\{0\}\right)$, oriented counterclockwise. For definiteness put $S_{j}^{1}=\left(\left\{z \in \mathbb{R}^{2}:\|z\|=1 /(3 n)\right\}+j / n\right) \times\{0\}$ and $C_{n}=\bigcup_{j=1}^{n} S_{j}^{1}$, which is therefore oriented. [3.16] Let Homeo $\left(D^{3}, C_{n}\right)$ be the group of orientation preserving homeomorphisms $D^{3} \rightarrow D^{3}$, which restrict to an orientation preserving homeomorphism $C_{n} \rightarrow C_{n}$, and which are the identity over $\partial D^{3}$. The group operation in $\operatorname{Homeo}\left(D^{3}, C_{n}\right)$ is composition. Two homeomorphisms $f, g$ : $\operatorname{Homeo}\left(D^{3}, C_{n}\right)$ are said to be pair-isotopic, if there exists a map $H: D^{3} \times[0,1] \rightarrow D^{3}$ such that $(z, t) \in D^{3} \mapsto H(z, t, s) \in D^{3}$ is in $\operatorname{Homeo}\left(D^{3}, C_{n}\right)$, for each $s \in[0,1]$.

Definition 16. Let $\operatorname{MCG}\left(D^{3}, C_{n}\right)$ (the "mapping class group of $\left(D^{3}, C_{n}\right)$ ") be the group of homeomorphisms $f \in \operatorname{Homeo}\left(D^{3}, C_{n}\right)$, considered up to pair isotopy. The group law in $\operatorname{MCG}\left(D^{3}, C_{n}\right)$ is induced by the composition in $\operatorname{Homeo}\left(D^{3}, C_{n}\right)$, which descends to the quotient under pair isotopy. In this paper, $\operatorname{MCG}\left(D^{3}, C_{n}\right)$ is also denoted $\mathrm{LB}_{n}$ and called the "loop braid group (in $n$ circles)".

As we shall illustrate shortly, $\mathrm{MCG}\left(D^{3}, C_{n}\right)$ can be thought of as a group of braidings of loop worldsheets in (3+1)-dimensions, generalising the ordinary braid group regarded as a group of braidings of point world-lines in (2+1)-dimensions; see [13, 14. Hence the term 'loop braid group' and the notation $\mathrm{LB}_{n}$.

For some manipulations, and for example representation theoretically, the given realisation of $\mathrm{LB}_{n}$ is difficult to work with. Next we prepare a useful presentation.

Theorem 17 (Baez et al [13, Damiani [14]). There exists an isomorphism:

$$
T: B \in \mathrm{WB}_{n} \longmapsto T_{B} \in \operatorname{MCG}\left(D^{3}, C_{n}\right)=\mathrm{LB}_{n} .
$$

This isomorphism sends a group generator [3.12] $g \in \mathrm{WB}_{n}$ of the form $S_{a}^{+}[n]$ or $V_{a}[n]$, to the pair-isotopy class of the homeomorphism $f^{g}=\phi_{t=1}^{g}:\left(D^{3}, C_{n}\right) \rightarrow\left(D^{3}, C_{n}\right)$ at the end of the isotopies $\Phi^{g}=(t \in[0,1] \mapsto$ $\left.\phi_{t}^{g} \in \operatorname{Homeo}\left(D^{3}, \emptyset\right)\right)$, of $D^{3}$, relative to its boundary, indicated in the Fig. 5, below (only a few virtual braid strands and circles are displayed, but the general picture should be clear).

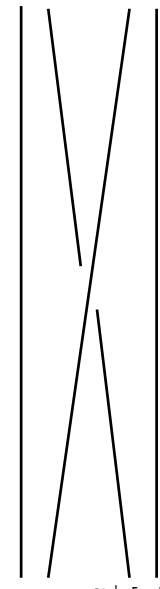

$g=S_{a}^{+}[n]$

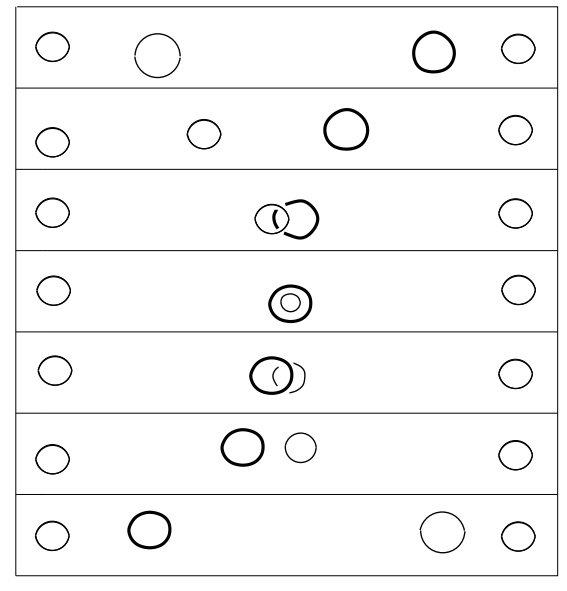

$\Phi^{g}$

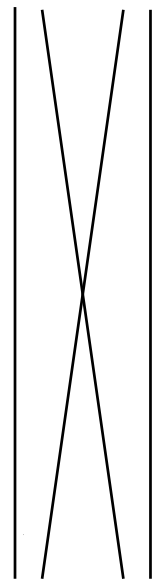

$g=V_{a}[n]$

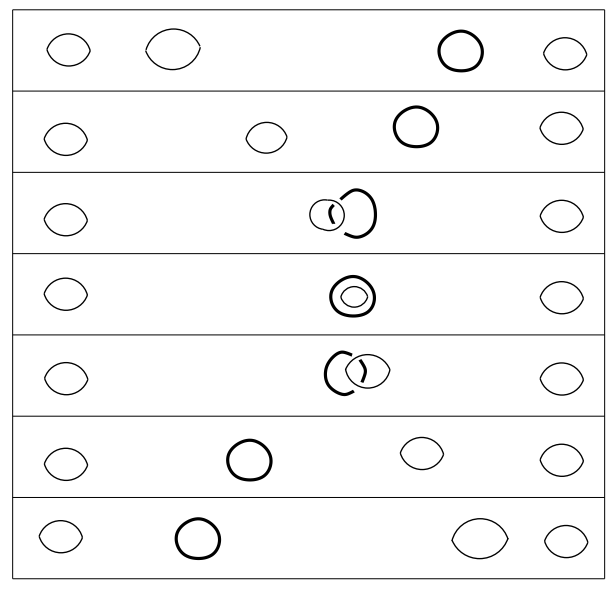

$\Phi^{g}$

Figure 5: A generator $g \in \mathrm{WB}_{n}$ gives rise to an isotopy $\Phi^{g}=\left(t \in[0,1] \mapsto \phi_{t}^{g} \in \operatorname{Homeo}\left(D^{3}, \emptyset\right)\right)$ of $D^{3}$.

We note that the convention for loop braiding in Fig. 5 is opposite to that in Equations (34)-(35) in 92 ,

This theorem was stated in [13] for smooth motion groups. The interpretation of smooth motion groups in terms of topological mapping class groups appears in [14]. In order to connect the two points of view, we can use results in [15, to pass from mapping class groups to motion groups, and [84, to go from the continuous setting to the smooth setting. In [67] it is proven that $\mathrm{WB}_{n}$ has another topological realisation as the fundamental group of the configuration space of rings in $D^{3}$.

Several other groups are isomorphic to $\mathrm{WB}_{n}$, e.g. the group of conjugating automorphisms of a free group [85] - we will go back to this in 4.5.1. For further discussion see [14]. 


\section{Bikoids and welded bikoids (W-bikoids)}

\subsection{The wreath product groupoid $\Gamma^{2 n}=\Gamma^{n} \rtimes \Sigma_{n}$ of a groupoid $\Gamma$}

Let $\Gamma=\left(\Gamma_{1}, \Gamma_{0}, \sigma, \tau, \iota, \star\right)$ be a groupoid. For $n \in \mathbb{Z}^{+}$, we write $\left(x_{1}, \ldots, x_{n}\right)=\underline{x} \in \Gamma_{0}^{n}$ and $\left(\gamma_{1}, \ldots, \gamma_{n}\right)=\underline{\gamma} \in$ $\Gamma_{1}^{n}$. The product groupoid $\Gamma^{n}$ is $\Gamma^{n}=\left(\Gamma_{1}^{n}, \Gamma_{0}^{n}, \underline{\sigma}, \underline{\tau}, \underline{\iota}, \underline{\star}\right)$, with product composition. Morphisms in $\Gamma^{n} \bar{c}^{-}$ be represented in a variety of ways, as indicated below:

$$
(\underline{\gamma}: \underline{x} \rightarrow \underline{y})=(\underline{x} \stackrel{\gamma}{\rightarrow} \underline{y})=\left(\left(x_{1}, \ldots, x_{n}\right) \stackrel{\left(\gamma_{1}, \ldots, \gamma_{n}\right)}{\longrightarrow}\left(y_{1}, \ldots, y_{n}\right)\right)=\left(\left(x_{1} \stackrel{\gamma_{1}}{\longrightarrow} y_{1}\right) \otimes \ldots \otimes\left(x_{n} \stackrel{\gamma_{n}}{\longrightarrow} y_{n}\right)\right) .
$$

[4.1] Cf. [3.4] for our convention on the symmetric group $\Sigma_{n}$. We have a left-action of $\Sigma_{n}$ on $\Gamma^{n}$ by groupoid functors, obtained by permuting components. Namely if $f \in \Sigma_{n}$ and $(\underline{\gamma}: \underline{x} \rightarrow \underline{z}) \in \Gamma_{1}^{n}$, we put:

$$
\begin{aligned}
f \triangleright\left(\left(x_{1}, x_{2}, \ldots x_{n}\right) \stackrel{\left(\gamma_{1}, \gamma_{2}, \ldots, \gamma_{n}\right)}{\longrightarrow}\left(z_{1}, z_{2}, \ldots z_{n}\right)\right)=\left(f \triangleright\left(x_{1}, x_{2}, \ldots x_{n}\right) \stackrel{f \triangleright\left(\gamma_{1}, \gamma_{2}, \ldots, \gamma_{n}\right)}{\longrightarrow} f \triangleright\left(z_{1}, z_{2}, \ldots z_{n}\right)\right) \\
\doteq\left(\left(x_{f(1)}, x_{f(2)}, \ldots x_{f(n)}\right) \stackrel{\left(\gamma_{f(1)}, \gamma_{f(2)}, \ldots, \gamma_{f(n)}\right)}{\longrightarrow}\left(z_{f(1)}, z_{f(2)}, \ldots z_{f(n)}\right)\right) .
\end{aligned}
$$

Definition 18. The 'wreath product groupoid' $\Gamma^{2 n}=\Gamma^{n} \rtimes \Sigma_{n}$, with respect to the action in [4.1] is defined as follows. The set of objects is $\Gamma_{0}^{n}$ and the set of morphisms is $\Gamma_{1}^{n} \times \Sigma_{n}$. Arrows have the form:

$$
\left(\left(\sigma\left(\gamma_{1}\right), \ldots, \sigma\left(\gamma_{n}\right)\right) \stackrel{\left(\left(\gamma_{1}, \ldots, \gamma_{n}\right), f\right)}{\longrightarrow}\left(\tau\left(\gamma_{f^{-1}(1)}\right), \ldots, \tau\left(\gamma_{f^{-1}(n)}\right)\right)\right)=\left(\underline{\sigma}(\underline{\gamma}) \stackrel{(\underline{\gamma}, f)}{\longrightarrow} f^{-1} \triangleright(\underline{\tau}(\underline{\gamma})) .\right.
$$

And given another arrow in $\Gamma^{2 n}$, namely $\left(\underline{\sigma}\left(\underline{\phi} \stackrel{(\underline{\phi}, g)}{\longrightarrow} g^{-1} \triangleright \underline{\tau}(\underline{\phi})\right)\right.$, with $\underline{\sigma}(\underline{\phi})=f^{-1} \triangleright \underline{\tau}(\underline{\gamma})$, the composition is:

$$
\left(\underline{\sigma}(\underline{\gamma}) \stackrel{(\underline{\gamma}, f)}{\longrightarrow} f^{-1} \triangleright \underline{\tau}(\underline{\gamma})\right) \star\left(f^{-1} \triangleright \underline{\tau}(\underline{\gamma}) \stackrel{(\underline{\phi}, g)}{\longrightarrow} g^{-1} \triangleright \underline{\tau}(\underline{\phi})\right)=\left(\underline{\sigma}(\underline{\gamma}) \stackrel{(\underline{\gamma} \star(f \triangleright \underline{\phi}), f \cdot g)}{\longrightarrow} g^{-1} \triangleright \underline{\tau}(\underline{\phi})\right) .
$$

(These apparently awkward conventions are justified by the graphical calculus in 44.2 .)

\subsection{Graphical calculus for $\Gamma^{n} \rtimes \Sigma_{n}=\Gamma^{2 n}$}

Recall [3.11] and 3.14] Any welded (or virtual) braid [Q], and any virtual braid diagram $Q$, gives rise to a permutation $U_{Q}$. Hence permutations can be represented by virtual braids diagrams. Thus we can use a graphical calculus to represent morphisms in $\Gamma^{n} \rtimes \Sigma_{n}$, cf. [86]. We explain the graphical calculus for $n=3$ (the other cases extend in the obvious way). This provides visual help for some calculations in this paper.

Firstly note that bijections $f:\{1,2,3\} \rightarrow\{1,2,3\}$ can be represented by virtual braid diagrams as below:
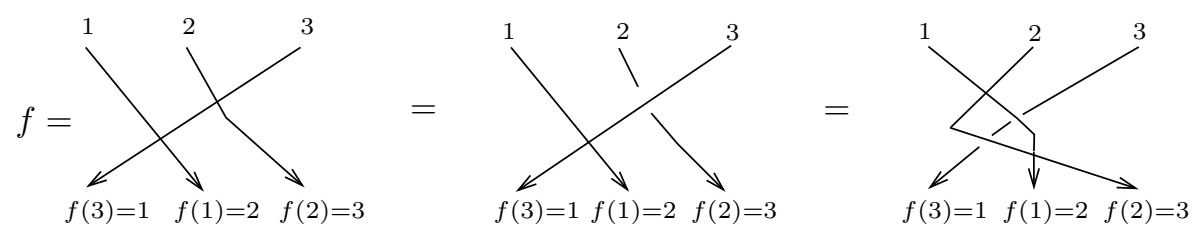

Here only initial and end-points of strands matter. In this diagrammatic presentation, the product $f . g$ of two permutations is given by the vertical juxtaposition where $f$ stays on top of $g$. (In order for this to work, we must use convention [3.4] for the symmetric group.)

The morphisms of $\Gamma^{3} \rtimes \Sigma_{3}$ can be represented by diagrams as in Fig. 6, Only initial and end-points of strands matter, encoding permutation components of morphisms in $\Gamma^{3} \rtimes \Sigma_{3}$. Hence, we can switch positive crossings with negative or virtual crossings without changing the morphism in $\Gamma^{3} \rtimes \Sigma_{3}$ that we are describing. 


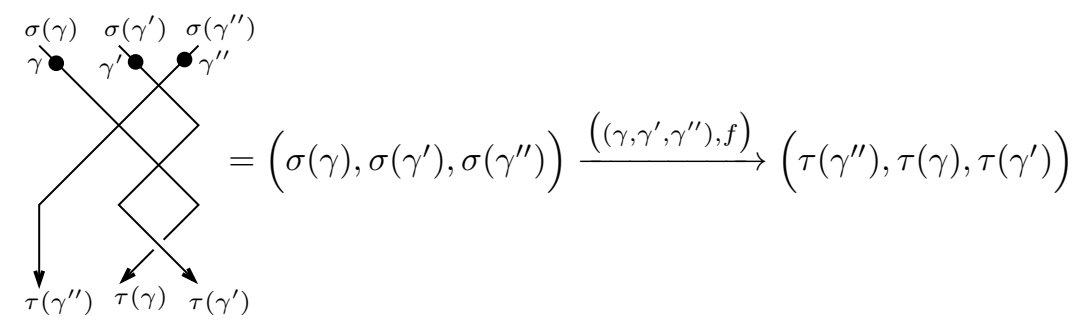

Figure 6: A morphism in $\Gamma^{3} \rtimes \Sigma_{3}$. Here $f \in \Sigma_{3}$ is such that $f(1)=2, f(2)=3$ and $f(3)=1$.

For $\gamma=$ id we may omit this and the blob "•" entirely.

Assume that two morphisms in $\Gamma^{3} \rtimes \Sigma_{3}$ are composable. (In the case below this means $\sigma(\phi)=\tau\left(\gamma^{\prime \prime}\right)$, $\sigma\left(\phi^{\prime}\right)=\tau\left(\gamma^{\prime}\right)$, and $\sigma\left(\phi^{\prime \prime}\right)=\tau(\gamma)$.) Graphically their composition is via vertical juxtaposition, as below:

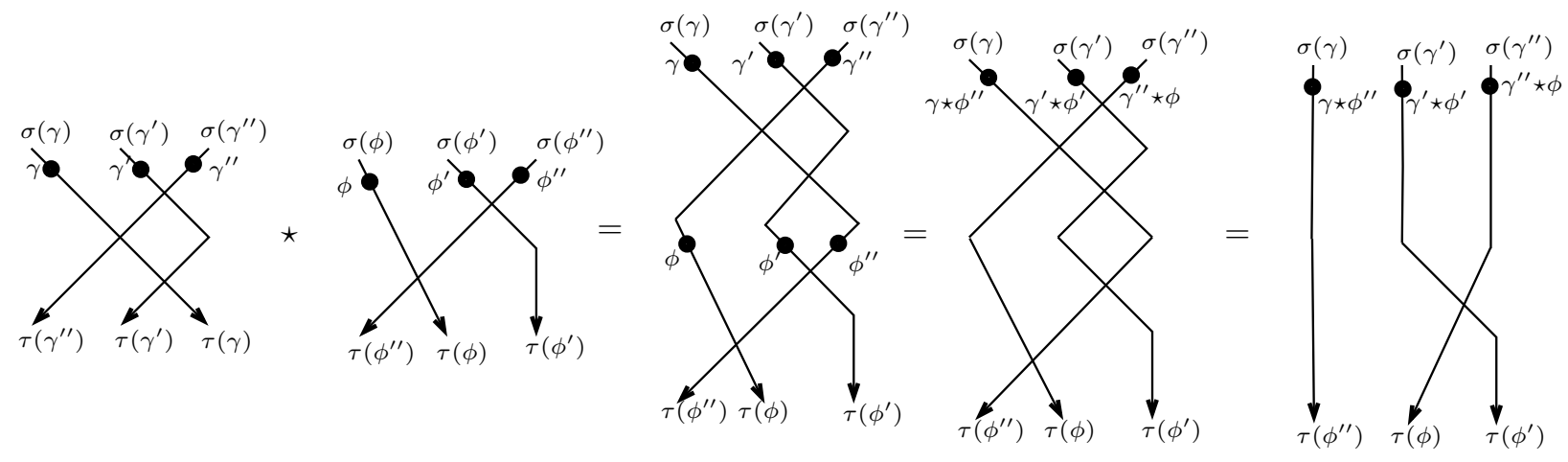

Figure 7: Graphical notation for composing morphisms in $\Gamma^{3} \rtimes \Sigma_{3}$.

Hence, blobs in a diagram can be moved freely along a strand, including pass a crossing, as long as no other blob gets in the way. We can compose the arrows colouring any two contiguous blobs in the same strand.

\subsection{Conventions and notation for biracks and welded biracks (W-biracks)}

References on quandles, biquandles and biracks include: [22, 26, 27, 29, 35, 36, 37, 87, 88.

Definition 19 (Birack, W-birack). A birack $(X, /, \backslash)$ is a set $X$ with two maps $X \times X \rightarrow X$, denoted $(x, y) \mapsto x \backslash y$, called " $x$ under $y$ ", and $(x, y) \mapsto y / x$ called " $y$ over $x$ ". The conditions to be satisfied are:

1. Fixing $a \in X$, the maps $f_{a}, f^{a}: X \rightarrow X$ given by $f_{a}: x \mapsto x / a$ and $f^{a}: x \mapsto x \backslash a$ each are invertible.

2. The map $S: X \times X \rightarrow X \times X$ given by $(x, y) \mapsto(y / x, x \backslash y)$ (called [26] a "switch") is invertible.

3. Putting $S_{1}=S \times \operatorname{id}_{X}: X \times X \times X \rightarrow X \times X \times X$ and $S_{2}=\operatorname{id}_{X} \times S$ then $S_{1} \circ S_{2} \circ S_{1}=S_{2} \circ S_{1} \circ S_{2}$.

A birack is "welded", or a $W$-birack, if for each $x, y, z$ the following hold:

$$
(z / x) / y=(z / y) / x, \quad y \backslash z=y \backslash(z / x)), \quad x \backslash z=x \backslash(z / y) .
$$

The last equation is of course redundant. It is left since it facilitates some of the diagrammatic proofs later.

[4.2] Let $G$ be a group. The operations $h / g=h$ and $g \backslash h=h^{-1} g h$ make $G$ a W-birack (also a quandle [26]). [4.3] For any birack $X$, we may visualize the maps $S$ and $t_{1,2}: X^{2} \rightarrow X^{2}$ given by $t_{1,2}:(x, y) \mapsto(y, x)$ as sending $(x, y) \in X \times X$ to the pairs appearing at the bottom of the diagrams:

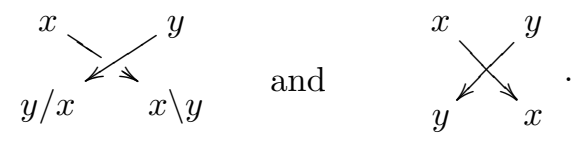


In particular $S_{1} \circ S_{2} \circ S_{1}=S_{2} \circ S_{1} \circ S_{2}$ becomes identification of the bottom rows here, for each $x, y, z \in X$ :
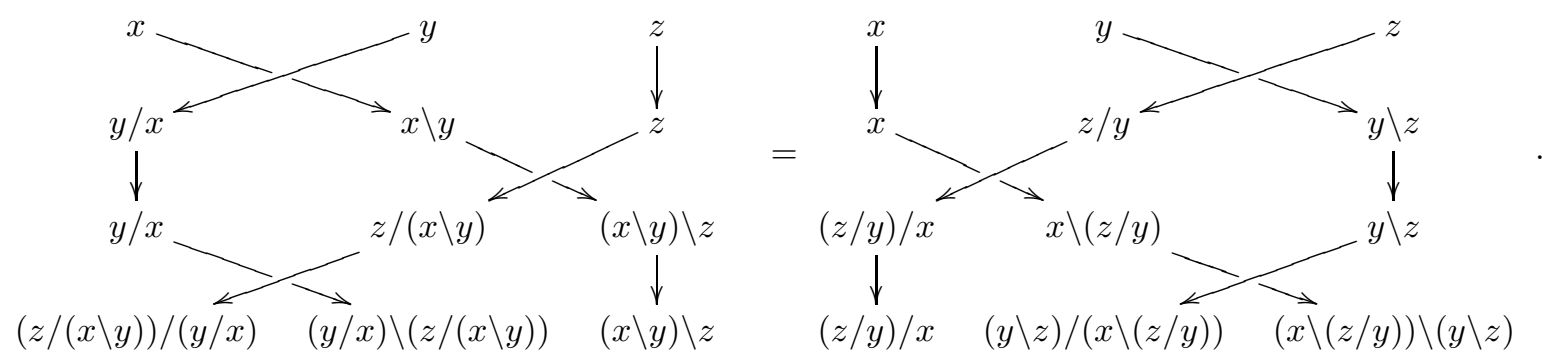

The welded birack axiom (53) says that the bottom colours of the diagrams below coincide, if $x, y, z \in X$ :
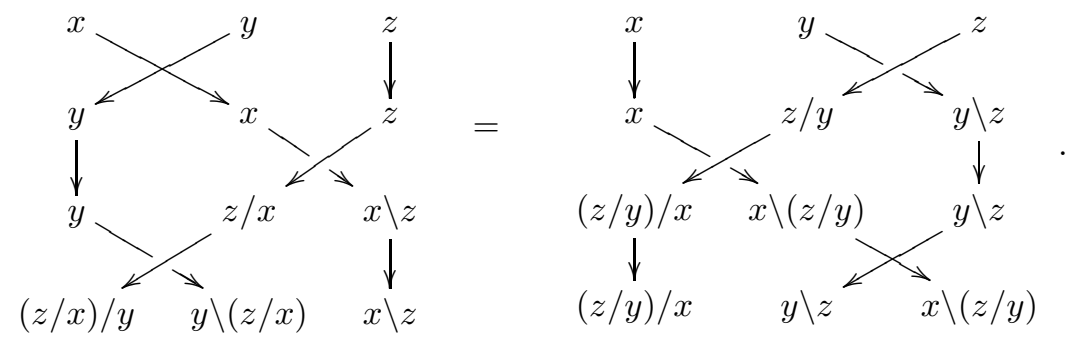

Note that the bottom colours in (56) coincide identically, given any triple $(x, y, z) \in X^{3}$ :
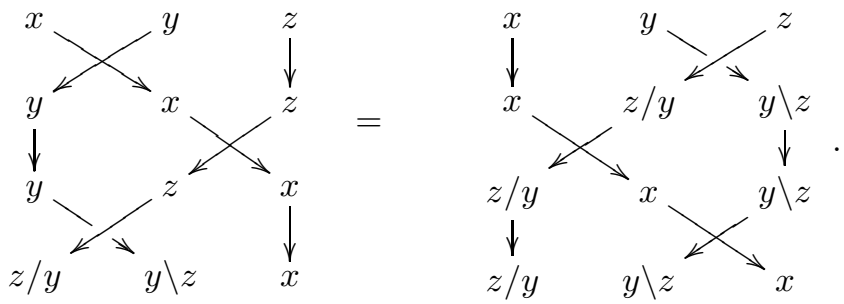

(See 93.3 , ) The following is standard [26, 27, 29, 22. In this paper we will extend this to bikoids.

Lemma 20. If $(X, /, \backslash)$ is a birack, then $X^{n}=X \times \cdots \times X$ has a right-action " $\triangleleft$ " of $\mathrm{VB}_{n}$ given by:

$$
\begin{aligned}
\left(x_{1}, \ldots, x_{a-1}, x_{a}, x_{a+1}, x_{a+2}, \ldots, x_{n}\right) \triangleleft S_{a}^{+}[n] & =\left(x_{1}, \ldots, x_{a-1}, x_{a+1} / x_{a}, x_{a} \backslash x_{a+1}, x_{a+2}, \ldots x_{n}\right), \\
\left(x_{1}, \ldots, x_{a-1}, x_{a}, x_{a+1}, x_{a+2}, \ldots, x_{n}\right) \triangleleft V_{a}[n] & =\left(x_{1}, \ldots, x_{a-1}, x_{a+1}, x_{a}, x_{a+2}, \ldots x_{n}\right) .
\end{aligned}
$$

This action descends to an action of the welded braid group $\mathrm{WB}_{n}$ if, and only if, $(X, /, \backslash)$ is welded.

Proof. We follow the formulation in [3.12] for $\mathrm{VB}_{n}$. Firstly $(-) \triangleleft S_{a}^{+}[n]$ is invertible by item 2 of Def. 19. Then note that $\underline{x} \triangleleft S_{a}^{+}[n]$ and $\underline{x} \triangleleft V_{a}[n]$, where $\underline{x}=\left(x_{1}, \ldots, x_{a-1}, x_{a}, x_{a+1}, x_{a+2}, \ldots, x_{n}\right)$, are the bottom colours of the following diagrams:

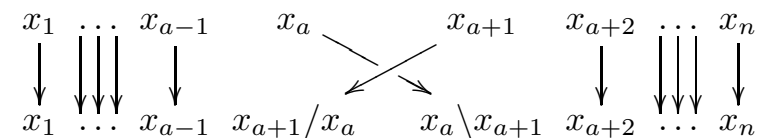

and

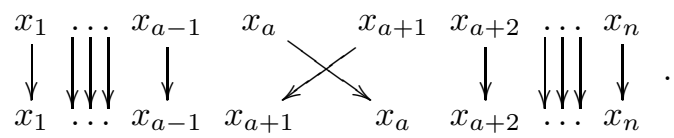

The check of RIII (46) from Def. 11 boils down to (54); VRIII (48) boils down to (56). Equations (44), (45) and (47) are trivial. Equation (49) follows from (55), which happens if, and only if, $(X, /, \backslash)$ is welded.

[4.4] By extending linearly, given a birack, we hence have a right representation $\triangleleft$ of $\mathrm{VB}_{n}$ on the vector space $\kappa\left(X^{n}\right) \cong \kappa(X)^{n \otimes}$. Here $\kappa$ is a field. This descends to a representation of $\mathrm{WB}_{n}$ if $(X, /, \backslash)$ is welded.

We will be particularly interested in biracks for which the reverse form of the welded Reidemeister III move in (50) does not hold for the action $\triangleleft$ (Lem. 20) of $\mathrm{WB}_{n}$ on $X^{n}$. These are called essential biracks. 
Definition 21. (We follow [22].) A W-birack $(X, /, \backslash)$ is called essential if it does not hold that:

$$
\forall x, y, z \in X^{3}:(x \backslash y) \backslash z=(x \backslash z) \backslash y, \quad \text { and } \quad y / x=y /(x \backslash z), \quad \text { and } \quad z /(x \backslash y)=z / x .
$$

[4.5] Essential W-biracks are the most interesting when it comes to applications to $\mathrm{WB}_{n}$. This is because otherwise $\triangleleft$ in Lem. 20] will descend to a representation of the unrestricted virtual braid group; see Rem. 14 .

\subsection{Bikoids: definition}

Definition 22 (Bikoid). A bikoid $\left(\Gamma, X^{+}\right)$is a groupoid $\Gamma=\left(\Gamma_{1}, \Gamma_{0}, \sigma, \tau, \iota, \star\right)$, together with set maps $L, R: \Gamma_{0} \times \Gamma_{0} \rightarrow \Gamma_{1}$, such that the following hold. Firstly, $\sigma(L(x, y))=x, \sigma(R(x, y))=y$. Secondly, $L$ and $R$ define a birack $\left(\Gamma_{0}, /, \backslash\right)$, called the underlying birack of $\left(\Gamma, X^{+}\right)$, by $x \backslash y=\tau(L(x, y))$ and $y / x=\tau(R(x, y))$. That is, the source and target of $L(x, y)$ and $R(x, y)$ are as indicated below:

$$
(x \stackrel{L(x, y)}{\longrightarrow} x \backslash y) \text { and }(y \stackrel{R(x, y)}{\longrightarrow} y / x) .
$$

Next define the following morphisms of the wreath product $\Gamma^{2} \rtimes \Sigma_{2}=\Gamma^{2}$ (recall [3.13], 4.1] and 44.2):

$$
\begin{aligned}
& Y^{+}(x, y)=\left(x \otimes y \stackrel{\left((L(x, y), R(x, y)), 1_{\Sigma_{2}}\right)}{\longrightarrow} x \backslash y \otimes y / x\right), \\
& X^{+}(x, y)=Y^{+}(x, y) \star\left(x \backslash y \otimes y / x \stackrel{\left.\left(\operatorname{(id}_{x \backslash y}, \mathrm{id}_{y / x}\right), t_{1,2}^{2}\right)}{\longrightarrow} y / x \otimes x \backslash y\right) \\
& =\left(x \otimes y \stackrel{\left((L(x, y), R(x, y)), t_{1,2}^{2}\right)}{\longrightarrow} y / x \otimes x \backslash y\right) .
\end{aligned}
$$

Note $\underline{\sigma}\left(X^{+}(x, y)\right)=(x, y)=x \otimes y$. We use the notations (recall the diagrammatic calculus for $\Gamma^{22}$ in 4.2 ):

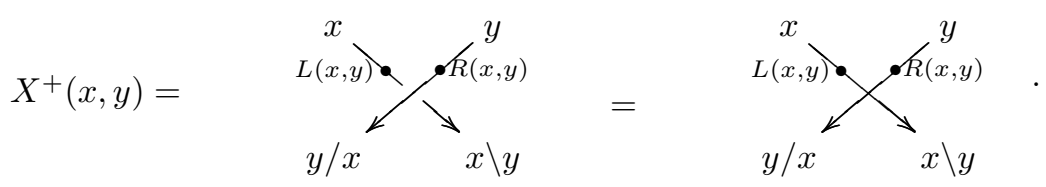

We impose that for each $x, y, z \in \Gamma_{0}$ the equation below holds in $\Gamma^{3} \rtimes \Sigma_{3}=\Gamma^{23}$ :

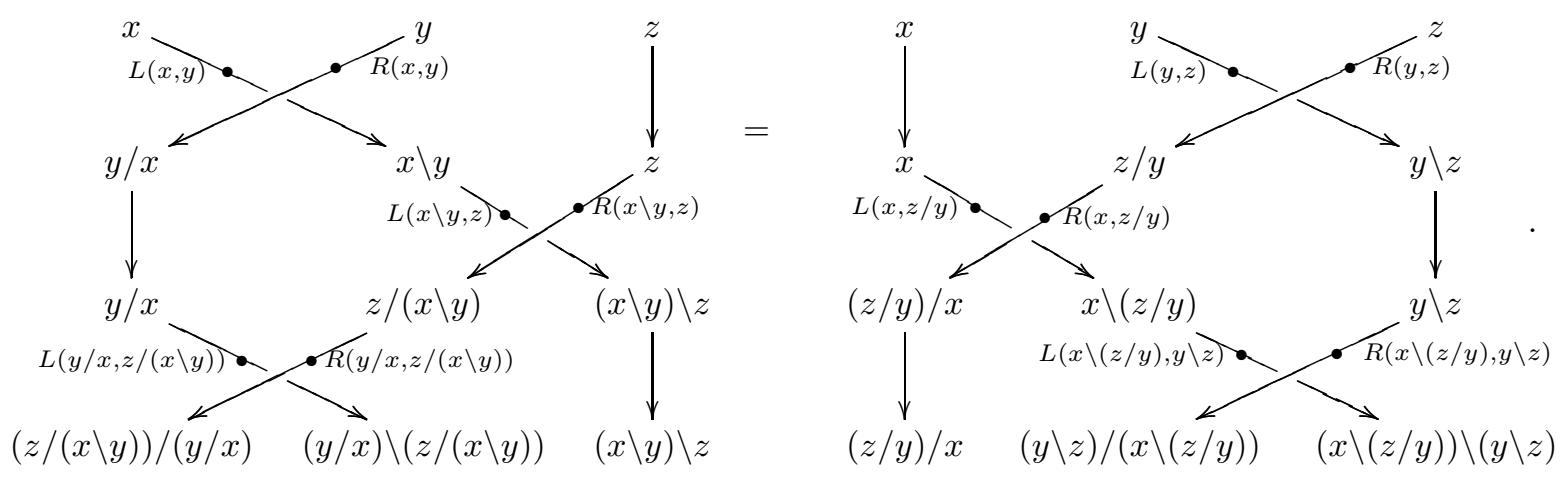

Recall that undecorated lines carry identities in $\Gamma$. Hence Equation (61) means that for each $x, y, z \in \Gamma_{0}$ :

$$
\begin{aligned}
L(x, y) \star L(x \backslash y, z) & =L(x, z / y) \star L(x \backslash(z / y), y \backslash z), \\
R(x, y) \star L(y / x, z /(x \backslash y)) & =L(y, z) \star R(x \backslash(z / y), y \backslash z), \\
R(x \backslash y, z) \star R(y / x, z /(x \backslash y)) & =R(y, z) \star R(x, z / y) .
\end{aligned}
$$

Remark 23 (Holonomy arrows). The $L(x, y)$ and $R(x, y)$ arrows are called holonomy arrows. In the context of finite group and finite 2-group topological field theory, they encode Aharonov-Bohm phases [3, 42, 43. arising from flat connection holonomy and flat 2-connection 2-dimensional holonomy obtained when pointparticles move in 2-dimensional space and loop-particles move in 3-dimensional space - see 2.1 and $\$ 2.3$, 
Remark 24. We can define a bikoid $\left(\Gamma, X^{+}\right)$as being given by a birack structure on $\Gamma_{0}$, together with morphisms $(L(x, y): x \rightarrow x \backslash y)$ and $(R(x, y): y \rightarrow y / x)$, in $\Gamma$, for each $x, y \in \Gamma_{0}$, satisfying Equation (62). The wreath groupoid approach chosen, and its graphical calculus, greatly facilitates the proofs to come.

[4.6] Let $\left(\Gamma, X^{+}\right)$be a bikoid. Consider the underlying birack $\left(\Gamma_{0}, /, \backslash\right)$ of $\left(\Gamma, X^{+}\right)$. In particular the map $(x, y) \in \Gamma_{0} \times \Gamma_{0} \mapsto(y / x, x \backslash y) \in \Gamma_{0} \times \Gamma_{0}$ is bijective (Def. 19). We put (the inverse is taken in $\left.\Gamma^{2} \rtimes \Sigma_{2}\right)$ :

$$
X^{-}(y / x, x \backslash y)=\left((x, y) \stackrel{X^{+}(x, y)}{\longrightarrow}(y / x, x \backslash y)\right)^{-1}=\left((y / x, x \backslash y) \stackrel{X^{-}(y / x, x \backslash y)}{\longrightarrow}(x, y)\right) \in \operatorname{Mor}\left(\Gamma^{2} \rtimes \Sigma_{2}\right) .
$$

We use the following diagrammatic notations for $X^{-}(y / x, x \backslash y)$ :

$$
X^{-}(y / x, x \backslash y)=
$$

[4.7] Thus $\overline{L(x, y)} \star L(x, y)=\operatorname{id}_{x \backslash y}$ and $L(x, y) \star \overline{L(x, y)}=\mathrm{id}_{x}$. And the same for $R(x, y)$ and $\overline{R(x, y)}$.

Definition 25 (Welded bikoid). (Recall Def. 19 and Equation (55).) A bikoid $\left(\Gamma, X^{+}\right)$is called welded, or a $W$-bikoid, if $\left(\Gamma_{0}, /, \backslash\right)$ is a welded birack, and for each $x, y, z \in \Gamma_{0}$ it holds that:

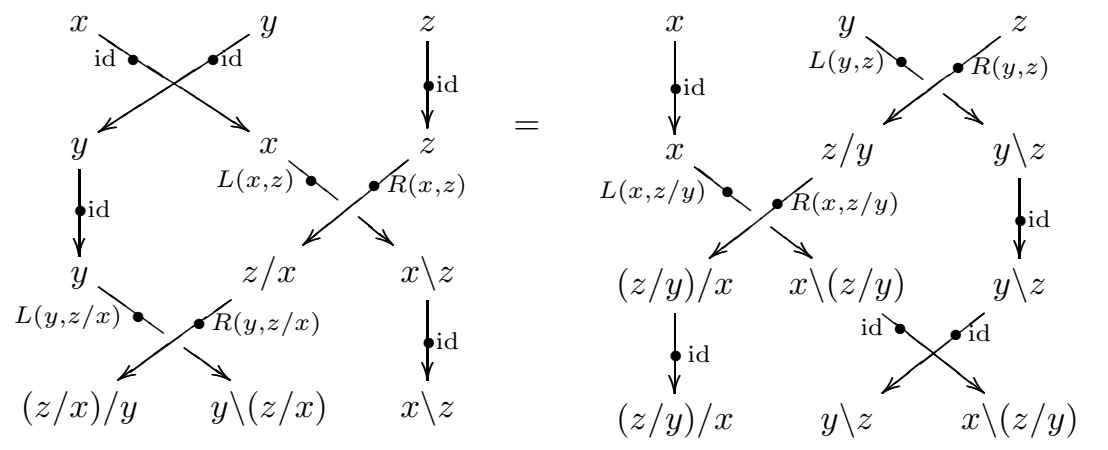

Since $\left(\Gamma_{0}, /, \backslash\right)$ is a W-birack, the equation above means that for each $x, y, z \in \Gamma_{0}$ we have that:

$$
L(x, z)=L(x, z / y), \quad L(y, z)=L(y, z / x), \quad R(x, z) \star R(y, z / x)=R(y, z) \star R(x, z / y) .
$$

Proposition 26 (Finite group W-bikoid). Let $G$ be a group. We have a W-bikoid structure $X_{G}^{+}$in the groupoid $\operatorname{AUT}(G)$ of Example 3 . The underlying birack $\left(\Gamma_{0}, /, \backslash\right)=(G, /, \backslash)$ is given by $y / x=y$ and $x \backslash y=y^{-1} x y$, cf. [4.2]. The holonomy morphisms are as follows (below we put $\bar{y}=y^{-1}$, where $y \in G$ ):

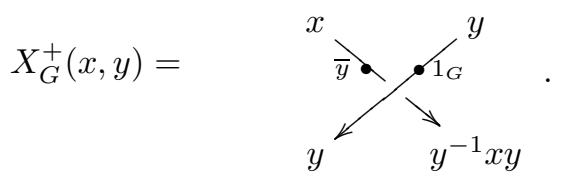

Proof. Recalling [4.2], $(G, /, \backslash)$ is a W-birack. Equation (61) - equivalently (62) - is true since:
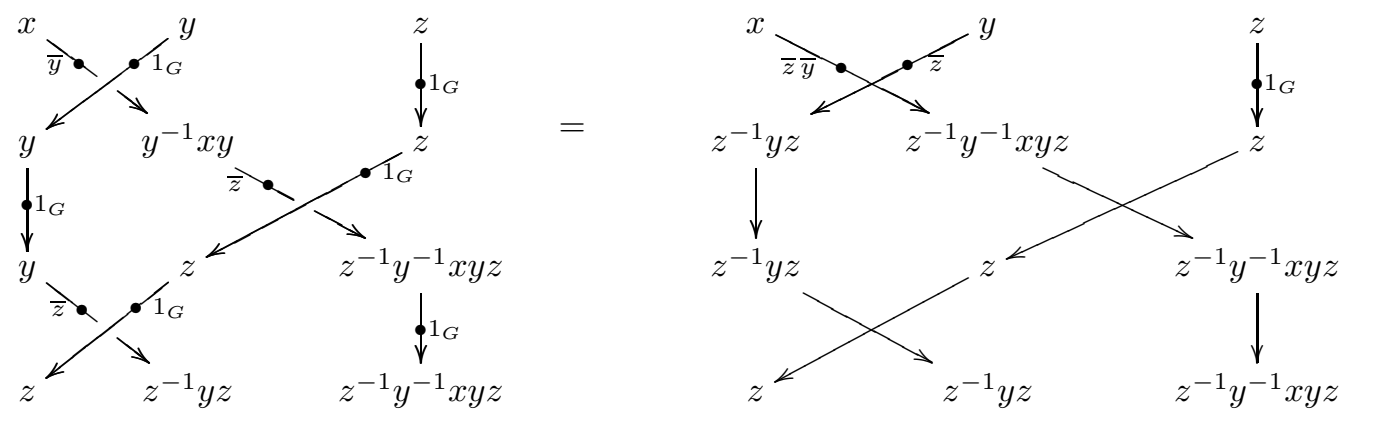
(Recall our convention for composition in $\operatorname{AUT}(G)$; see Example 3.) Whereas:
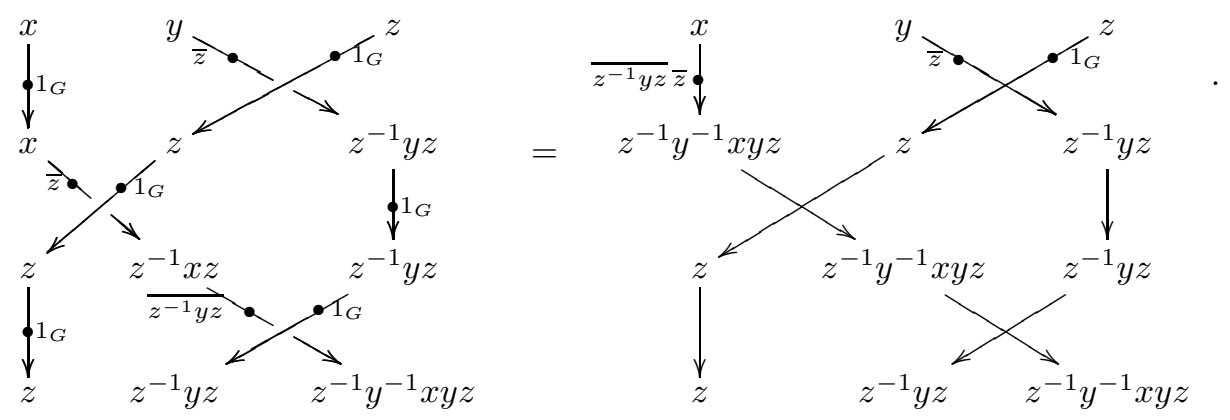

Since $\overline{z^{-1} y z} \bar{z}=z^{-1} y^{-1} z z^{-1}=\bar{z} \bar{y}$, the morphisms in $\Gamma^{23}$ in the right-hand-sides of (66) and (67) coincide. That the bikoid $X_{G}^{+}$is welded is proved similarly; we will prove this in a more general context in 86.2 .3 .

The W-bikoid $\left(\operatorname{AUT}(G), X_{G}^{+}\right)$is closely related to topological gauge theory in $D^{2}$; see $\$ 2.1$.

The proof of the following result is an easy exercise:

Proposition 27. Let $\left(\Gamma, X^{+}\right)$be a bikoid. Then $\Gamma_{1}$, the set of morphisms of $\Gamma$, is a birack with:

$$
\begin{aligned}
& \left(x^{\prime} \stackrel{\gamma}{\rightarrow} x\right) \backslash\left(y^{\prime} \stackrel{\phi}{\rightarrow} y\right)=\left(x^{\prime} \stackrel{\gamma}{\rightarrow} x\right) \star(x \stackrel{L(x, y)}{\longrightarrow} x \backslash y)=\left(x^{\prime} \stackrel{\gamma \star L(x, y)}{\longrightarrow} x \backslash y\right), \\
& \left(y^{\prime} \stackrel{\phi}{\rightarrow} y\right) /\left(x^{\prime} \stackrel{\gamma}{\rightarrow} x\right)=\left(y^{\prime} \stackrel{\phi}{\rightarrow} y\right) \star(y \stackrel{R(x, y)}{\longrightarrow} y / x)=\left(y^{\prime} \stackrel{\phi \star R(x, y)}{\longrightarrow} y / x\right) .
\end{aligned}
$$

Furthermore $\left(\Gamma, X^{+}\right)$is a welded bikoid if, and only if, $\left(\Gamma_{1}, /, \backslash\right)$ is a welded birack.

[4.8] To $\left(\Gamma_{1}, /, \backslash\right)$ we call the upper birack of $\left(\Gamma, X^{+}\right)$.

[4.9] By combining Prop. 27 with Lem. 20, we hence have a representation $\triangleleft^{*}$ of $\mathrm{VB}_{n}$ on $\mathbb{C} \Gamma_{1}^{n} \cong\left(\mathbb{C} \Gamma_{1}\right)^{n \otimes}$, derived from the upper birack of a bikoid $\left(\Gamma, X^{+}\right)$. Explicit formulae are in [1.8] The representation $\triangleleft^{*}$ of $\mathrm{VB}_{n}$ descends to a representation of $\mathrm{WB}_{n}$ when $\left(\Gamma, X^{+}\right)$is welded. This $\triangleleft^{*}$ will be generalised in $\$ 5$ ]

(Recall [4.5]). We will be particularly interested in essential W-bikoids. These are the W-bikoids for which the representation $\triangleleft^{*}$ does not descend from $\mathrm{WB}_{n}$ to the unrestricted virtual braid group in Rem. 14

Definition 28. A W-bikoid is called essential if its upper birack is essential (as in Def. 21).

Example 29. A quick calculation shows that if $G$ is non-abelian then the W-bikoid $X_{G}^{+}$in Prop. 26 is an essential W-bikoid. The lower birack of $X_{G}^{+}$, displayed in [4.2], may be non-essential when $G$ is non-abelian, if it happens that for all $x, y, z \in G$, it holds that $(x y) z(x y)^{-1}=(y x) z(y x)^{-1}$.

\subsection{Bikoids from abelian $g r$-groups}

There will be heavy use of semidirect products in this document. Here are our conventions.

[4.10] Let the group $G$ left-act by automorphisms on the group $E$. Such action is denoted by:

$$
(g, e) \in G \times E \mapsto g \triangleright e \in E .
$$

Our convention for the semidirect product $G \ltimes E=G \ltimes_{\triangleright} E$ is:

$$
(g, a)(h, b)=(g h, a g \triangleright b), \text { for each } g, h \in G \text { and } a, b \in E .
$$

Hence inverses in $G \ltimes_{\triangleright} E$ are: $(g, e)^{-1}=\left(g^{-1}, g^{-1} \triangleright e^{-1}\right)$.

[4.11] Let $g \in G$ and $e \in E$. We will frequently put $g=\left(g, 1_{E}\right)$, where $1_{E}$ is the identity of $E$, and $e=\left(1_{G}, e\right)$. Hence $(g, e)=e g=g\left(g^{-1} \triangleright e\right)$. Also recall that given left-actions $>$ of $G$ and of $E$ on a set $X$, then $(g, e) \gg x \doteq e \gg(g \gg x)$ is an action of $G \ltimes_{\triangleright} E$ on $X$ if, and only if, for each $g \in G, e \in E$ and $x \in X$ :

$$
e \gg(g \gg x)=g \gg\left(\left(g^{-1} \triangleright e\right) \gg x\right) .
$$


Definition 30 (Abelian gr-group). An abelian gr-group is given by pair $(G, A)$, or more correctly a triple $(G, A, \triangleright)$, where $G$ and $A$ are groups, with $A$ abelian, and $\triangleright$ is a left-action of $G$ on $A$ by automorphisms. Morphisms $\phi:(G, A) \rightarrow(H, B)$ of abelian gr-groups are defined as pairs of group maps $\phi_{1}: G \rightarrow H$ and $\phi_{2}: A \rightarrow B$ preserving group actions, namely $\phi_{2}(g \triangleright a)=\phi_{1}(g) \triangleright \phi_{2}(a)$, for each $g \in G$ and $a \in A$. The set of morphisms of abelian $g r$-groups between $(G, A)$ and $(H, B)$ is denoted $\operatorname{hom}_{g r}((G, A),(H, B))$.

Example 31. E.g., for $p$ a prime and $m \in \mathbb{Z}^{+}$, put $G=\mathrm{GL}\left(m, \mathbb{Z}_{p}\right)$, the group of invertible $m \times m$ matrices in the field $\left(\mathbb{Z}_{p},+, \times\right)$, and $A=\left(\mathbb{Z}_{p}^{m},+\right)$. The action is by matrix multiplication.

Let $(G, A)$ be an abelian $g r$-group, and $G \ltimes A=G \ltimes_{\triangleright} A$. Cf. Def. 3, let $\operatorname{TRANS}(G, A)=\operatorname{AUT}(G \ltimes A)$, the action groupoid (Def. 2) of the conjugation action of $G \ltimes A$ on itself. Thus arrows of $\operatorname{TRANS}(G, A)$ are:

$$
\left.\left((g, a) \stackrel{(w, k)}{\longrightarrow}(w, k)(g, a)(w, k)^{-1}\right)=\left(w g w^{-1}, k+w \triangleright a-\left(w g w^{-1}\right) \triangleright a\right)\right), \text { where } g, w \in G \text { and } a, k \in A .
$$

Theorem 32 (Abelian gr-group bikoids). We have a $W$-bikoid (TRANS $\left.(G, A), X_{g r}^{+}\right)$, given by:

$$
X_{g r}^{+}((z, a),(w, b))=\underbrace{(z, a)}_{\left(w, a+b-w^{-1} \triangleright a\right)}\left(w_{\left(w^{-1}, 0_{A}\right)}^{(w, b)}\left(w, w^{-1} \triangleright a\right)\right.
$$

Hence the underlying birack of $X_{g r}^{+}$is such that:

$$
(z, a) \backslash(w, b)=\left(w^{-1} z w, w^{-1} \triangleright a\right) \quad \text { and } \quad(w, b) /(z, a)=\left(w, a+b-w^{-1} \triangleright a\right) .
$$

We omit the proof, as we will treat a more general case (bikoids derived from crossed modules) in Thm. 57

Proposition 33. (Cf. Def. [28) The W-bikoid $X_{g r}^{+}$in (69) is essential if, and only if, it does not hold that:

$$
\forall y, z \in G, \forall a \in A: \quad y z=z y, \quad y^{-1} \triangleright a=\left(y^{-1} z^{-1}\right) \triangleright a, \quad \text { and } \quad z^{-1} \triangleright a=\left(z^{-1} y^{-1}\right) \triangleright a .
$$

Hence if $G$ is non-abelian, or if the action of $G$ on $A$ is non-trivial, then the $W$-bikoid $X_{g r}^{+}$in (69) is essential.

Proof. This follows by easy calculations.

Example 34. It is possible that $X_{g r}^{+}$in (69) is essential when $G$ is abelian. E.g. take $G=\mathbb{Z}_{2}=(\{1,-1\}, \times)$ and $A=\mathbb{Z}_{3}=(\{0,1,-1\},+)$. Hence $\mathbb{Z}_{2}$ acts on $\mathbb{Z}_{3}$ as $x \triangleright a=x a$. The associated W-bikoid is essential.

\subsubsection{Algebraic topological interpretation of abelian gr-group W-bikoids: 'balloons and hoops'}

We freely use 93.4 and Thm. 17. We use the same notation for $B \in \mathrm{WB}_{n}$ and its image $T_{B} \in \mathrm{LB}_{n}$.

Recall [32, 89] that the underlying birack [4.2] of the finite group bikoid (AUT $\left.(G), X_{G}^{+}\right)$of Prop. 26] essentially computes the cardinality of the set of group maps from the knot group of a welded knot into $G$.

Let $n \in \mathbb{Z}^{+}$. The underlying birack (70) of the abelian $g r$-group bikoid $X_{g r}^{+}=\left(\operatorname{TRANS}(G, A), X_{g r}^{+}\right)$of Thm. 32 is strongly related to the action of $\pi_{1}\left(D^{3} \backslash C_{n}, *\right)$ on $\pi_{2}\left(D^{3} \backslash C_{n}, *\right)$. We now explain this.

Consider the base point $*=(0,0,1) \in \partial\left(D^{3}\right)$ for $D^{3}$. Hence any homeomorphism $f:\left(D^{3}, C_{n}\right) \rightarrow\left(D^{3}, C_{n}\right)$ fixes $*$. So do the isotopies we consider. Homotopically, $\left(D^{3} \backslash C_{n}, *\right)$ is a wedge product of $n$ circles and $n$ 2-spheres. The fundamental group $\pi_{1}\left(D^{3} \backslash C_{n}, *\right)$ is the free product $\vee_{i=1}^{n} \mathbb{Z}_{i}$, where $\mathbb{Z}_{i}=\left\{m_{i}^{k}\right\}_{k \in \mathbb{Z}} \cong(\mathbb{Z},+)$. Here $m_{i} \in \pi_{1}\left(D^{3} \backslash C_{n}, *\right)$ is associated with the oriented unknotted circle $S_{i}^{1}$, in the usual way; see Fig. 8 , 


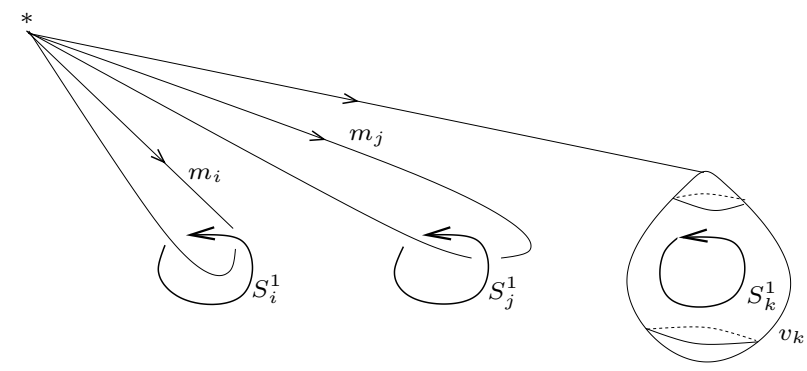

Figure 8: Elements $m_{i}, m_{j} \in \pi_{1}\left(D^{3} \backslash C_{n}, *\right), v_{k} \in \pi_{2}\left(D^{3} \backslash C_{n}, *\right)$ given by the unknotted circles $S_{i}^{1}, S_{j}^{1}, S_{k}^{1}$.

(This is the usual Wirtinger presentation of the fundamental group of a knot complement, see e.g. 90].) The group-algebra of $\pi_{1}\left(D^{3} \backslash C_{n}, *\right)$, with coefficients in $\mathbb{Z}$, is thus isomorphic to the algebra of Laurent polynomials $\mathbb{Z}\left\{m_{1}, m_{1}^{-1}, \ldots, m_{n}, m_{n}^{-1}\right\}$, in the non-commuting variables $m_{1}, \ldots, m_{n}$.

The second homotopy group $\pi_{2}\left(D^{3} \backslash C_{n}, *\right)$ is isomorphic to the second homology group of the universal cover of $D^{3} \backslash C_{n}$, which recall is homotopic to the wedge product of $n$ 1-spheres $S^{1}$ and $n$ 2-spheres $S^{2}$. Therefore $\pi_{2}\left(D^{3} \backslash C_{n}, *\right)$ is isomorphic to the free $\mathbb{Z}\left\{m_{1}, m_{1}^{-1}, \ldots, m_{n}, m_{n}^{-1}\right\}$-module on the variables $v_{1}, \ldots, v_{n}$; [91, Example 4.27] treats a particular case of this. Geometrically, each $v_{k} \in \pi_{2}\left(D^{3} \backslash C_{n}, *\right)$ traces a 'balloon' encircling the circle $S_{k}^{1}$; see Fig. 8. (A similar 'balloons and hoops' picture appears in [92.)

Given a path-connected pointed space $(X, *)$, the abelian gr-group made out of $\pi_{1}(X, *)$ acting on $\pi_{2}(X, *)$, in the usual way, is denoted by $\pi_{1,2}(X, *)$. The previous two paragraphs imply (given the freeness of $\pi_{1}\left(D^{3} \backslash C_{n}, *\right)$ and the freeness of $\pi_{2}\left(D^{3} \backslash C_{n}, *\right)$ as a $\mathbb{Z}\left(\pi_{1}\left(D^{3} \backslash C_{n}, *\right)\right)$-module) the following:

Lemma 35. Let $(G, A)$ be an abelian gr-group. Abelian gr-group maps $\phi=\left(\phi_{1}, \phi_{2}\right): \pi_{1,2}\left(D^{3} \backslash C_{n}, *\right) \rightarrow$ $(G, A)$ are in bijective correspondence with sequences of the form: $\left(\left(g_{1}, a_{1}\right), \ldots,\left(g_{n}, a_{n}\right)\right)$, where $g_{i} \in G$ and $a_{i} \in A$, for each $i=1, \ldots, n$. The bijection is defined by $g_{i}=\phi_{1}\left(m_{i}\right)$ and $a_{i}=\phi_{2}\left(v_{i}\right)$, for $i=1, \ldots, n$.

The loop braid group $\mathrm{LB}_{n}$ in 3.4 has a natural left-action $>$ in $\pi_{1,2}\left(D^{3} \backslash C_{n}, *\right)$ by abelian $g r$-group maps. In another language, we have a group map from $\mathrm{LB}_{n}$ to the automorphism group of the abelian gr-group $\pi_{1,2}\left(D^{3} \backslash C_{n}, *\right)$. This extends the embedding of the loop braid group into the automorphism group of a free group - the bit consisting of basis conjugating automorphisms - as explained in [14, §4] and [85.

Let us give details. A homeomorphism $f:\left(D^{3}, C_{n}\right) \rightarrow\left(D^{3}, C_{n}\right)$, considered up to pair-isotopy, functorially gives an isomorphism $f_{*}: \pi_{1,2}\left(D^{3} \backslash C_{n}, *\right) \rightarrow \pi_{1,2}\left(D^{3} \backslash C_{n}, *\right)$. And then given $m \in \pi_{1}\left(D^{3} \backslash C_{n}, *\right)$ and $v \in \pi_{2}\left(D^{3} \backslash C_{n}, *\right)$, we put $f>m=f_{*}(m)$ and $f>v=f_{*}(v)$. Hence $\operatorname{LB}_{n}$ right-acts in $\operatorname{hom}_{g r}\left(\pi_{1,2}\left(D^{3} \backslash\right.\right.$ $\left.\left.C_{n}, *\right),(G, A)\right)$, namely given $f \in \mathrm{LB}_{n}$, we send $\phi \in \operatorname{hom}_{g r}\left(\pi_{1,2}\left(D^{3} \backslash C_{n}, *\right),(G, A)\right)$ to $\phi \circ f_{*} \doteq \phi \lessdot f$.

Looking at Fig. 5 in Thm. 17, we can see that $S_{i}^{+}[n], V_{i}[n] \in \mathrm{LB}_{n}$ act in $\pi_{1,2}\left(D^{3} \backslash C_{n}, *\right)$ as:

$$
\begin{array}{lrr}
V_{i}[n] \gg m_{j}=m_{j}, \text { if } j \notin i, i+1, & V_{i}[n] \gg m_{(i+1)}=m_{i}, & V_{i}[n] \gg m_{i}=m_{(i+1)}, \\
V_{i}[n] \gg v_{j}=v_{j}, \text { if } j \notin i, i+1, & V_{i}[n] \gg v_{(i+1)}=v_{i}, & V_{i}[n] \gg v_{i}=v_{(i+1)}, \\
S_{i}^{+}[n] \gg m_{j}=m_{j}, \text { if } j \notin i, i+1, & S_{i}^{+}[n] \gg m_{(i+1)}=m_{i}, & S_{i}^{+}[n] \gg m_{i}=m_{i}^{-1} m_{i+1} m_{i}, \\
S_{i}^{+}[n] \gg v_{j}=v_{j}, \text { if } j \notin i, i+1, & & S_{i}^{+}[n] \gg v_{i}=m_{i}^{-1} \triangleright v_{(i+1)} .
\end{array}
$$

The hardest action to address is $S_{i}^{+}[n] \gg v_{(i+1)}$. Cf. the left bit of Fig. 5, when the circle $S_{i}^{i}$ goes inside the circle $S_{i+1}^{1}$, it 'drags' the balloon $v_{(i+1)}$ with it. Since the isotopy connecting the top and bottom of the left part of Fig 5 can be made local, the sum $v_{i}+v_{i+1}$ in $\pi_{2}\left(D^{3} \backslash C_{n}, *\right)$, which can be visualised as a large balloon encircling the circles $S_{i}^{1}$ and $S_{i+1}^{1}$, remains stable during the isotopy, since the isotopy can be made local enough so that it happens well inside the balloon $v_{i}+v_{i+1}$. In a more precise language, this means:

$$
S_{i}^{+}[n]>\left(v_{i}+v_{(i+1)}\right)=v_{i}+v_{(i+1)} .
$$

Since $S_{i}^{+}[n] \gg\left(v_{i}+v_{(i+1)}\right)=S_{i}^{+}[n] \gg v_{i}+S_{i}^{+}[n] \gg v_{(i+1)}$ and $S_{i}^{+}[n] \gg v_{i}=m_{i}^{-1} \triangleright v_{(i+1)}$, it follows that:

$$
S_{i}^{+}[n] \gg v_{(i+1)}=v_{i}+v_{(i+1)}-m_{i}^{-1} \triangleright v_{(i+1)} .
$$

Comparing with Equation (69), this implies, by noting that the $V_{a}[n]$ and the $S_{a}^{+}[n]$ generate $\mathrm{LB}_{n}$, that: 
Theorem 36 (Topological interpretation of abelian gr-group W-bikoids). Let $(G, A)$ be an abelian gr-group. Consider the right-action $\triangleleft$ of the loop braid group $\mathrm{LB}_{n} \cong \mathrm{WB}_{n}$ on $(G \times A)^{n}$ derived (Lem. (20) from the underlying birack (70) of the welded bikoid $X_{g r}^{+}$of (69). Looking at the elements in $(G \times A)^{n}$ as being abelian gr-group maps $\phi: \pi_{1,2}\left(D^{3} \backslash C_{n}, *\right) \rightarrow(G, A)$ - by Lem. 35 - then given a $B \in \mathrm{LB}_{n}$ it holds that $\phi \triangleleft B=\phi \circ B_{*}$, where $B_{*}: \pi_{1,2}\left(D^{3} \backslash C_{n}, *\right) \rightarrow \pi_{1,2}\left(D^{3} \backslash C_{n}, *\right)$ is the induced map on homotopy groups.

\section{$5 \quad$ Virtual and welded braid group representations from bikoids}

Throughout this section we will fix a groupoid $\Gamma$ and a bikoid $\left(\Gamma, X^{+}\right)$; see Def. 22 .

\subsection{Representations of $\mathrm{WB}_{n}$ derived from bikoids}

Recall the groupoid algebra $\mathbb{C}(\Gamma)$ in Def. 4. We define the following element $\mathcal{R} \in \mathbb{C}(\Gamma) \otimes \mathbb{C}(\Gamma)$ :

$$
\mathcal{R}=\sum_{x, y \in \Gamma_{0}}(x \stackrel{L(x, y)}{\longrightarrow} x \backslash y) \otimes(y \stackrel{R(x, y)}{\longrightarrow} y / x) .
$$

Lemma 37. The element $\mathcal{R}$ is invertible and its inverse is (recall the notation introduced in [3.7]):

$$
\mathcal{R}^{-1}=\sum_{a, b \in \Gamma_{0}}(a \backslash b \stackrel{\overline{L(a, b)}}{\longrightarrow} a) \otimes(b / a \stackrel{\overline{R(a, b)}}{\longrightarrow} b) .
$$

Moreover $\mathcal{R}$ satisfies the relation below:

$$
\mathcal{R}_{12} \mathcal{R}_{13} \mathcal{R}_{23}=\mathcal{R}_{23} \mathcal{R}_{13} \mathcal{R}_{12}, \text { in } \mathbb{C}(\Gamma) \otimes \mathbb{C}(\Gamma) \otimes \mathbb{C}(\Gamma) .
$$

Here $\mathcal{R}_{12}=\mathcal{R} \otimes \operatorname{id}_{\mathbb{C}(\Gamma)}, \mathcal{R}_{23}=\operatorname{id}_{\mathbb{C}(\Gamma)} \otimes \mathcal{R}$ and $\mathcal{R}_{13}=\sum_{x, y \in \Gamma_{0}}(x \stackrel{L(x, y)}{\longrightarrow} x \backslash y) \otimes \operatorname{id}_{\mathbb{C}(\Gamma)} \otimes(y \stackrel{R(x, y)}{\longrightarrow} y / x)$. Furthermore, if the bikoid $\left(\Gamma, X^{+}\right)$is welded, then in $\mathbb{C}(\Gamma) \otimes \mathbb{C}(\Gamma) \otimes \mathbb{C}(\Gamma)$ :

$$
\mathcal{R}_{13} \mathcal{R}_{23}=\mathcal{R}_{23} \mathcal{R}_{13}
$$

Note that Equation (73) is satisfied by R-matrices in quasi-triangular bialgebras; see e.g. [24, Thm.VIII.2.4]. Proof. We have:

$$
\begin{aligned}
\mathcal{R R}^{-1} & =\sum_{x, y, a, b \in \Gamma_{0}}((x \stackrel{L(x, y)}{\longrightarrow} x \backslash y) \otimes(y \stackrel{R(x, y)}{\longrightarrow} y / x))((a \backslash b \stackrel{\overline{L(a, b)}}{\longrightarrow} a) \otimes(b / a \stackrel{\overline{R(a, b)}}{\longrightarrow} b)) \\
& =\sum_{x, y, a, b \in \Gamma_{0}}((x \stackrel{L(x, y)}{\longrightarrow} x \backslash y)(a \backslash b \stackrel{\overline{L(a, b)}}{\longrightarrow} a)) \otimes((y \stackrel{R(x, y)}{\longrightarrow} y / x)(b / a \stackrel{\overline{R(a, b)}}{\longrightarrow} b)) \\
& \stackrel{(\# 1)}{=} \sum_{\substack{x, y, a, b \in \Gamma_{0} \\
x \backslash y=a \backslash b \wedge y / x=b / a}}((x \stackrel{L(x, y)}{\longrightarrow} x \backslash y)(a \backslash b \stackrel{\overline{L(a, b)}}{\longrightarrow} a)) \otimes((y \stackrel{R(x, y)}{\longrightarrow} y / x)(b / a \stackrel{\overline{R(a, b)}}{\longrightarrow} b)) \\
& \stackrel{(\# 2)}{=} \sum_{\substack{x, y, a, b \in \Gamma_{0} \\
x=a \wedge y=b}}((x \stackrel{L(x, y)}{\longrightarrow} x \backslash y)(a \backslash b \stackrel{\overline{L(a, b)}}{\longrightarrow} a)) \otimes((y \stackrel{R(x, y)}{\longrightarrow} y / x)(b / a \stackrel{\overline{R(a, b)}}{\longrightarrow} b)) \\
& \stackrel{(\# 3)}{=} \sum_{x, y \Gamma_{0}}\left(x \stackrel{\operatorname{id}_{x}}{\longrightarrow} x\right) \otimes\left(y \stackrel{\mathrm{id}_{y}}{\longrightarrow} y\right)=1_{\mathbb{C}(\Gamma)} \otimes 1_{\mathbb{C}(\Gamma) .}
\end{aligned}
$$

Here (\#1) follows from the definition of the product (42) in $\mathbb{C}(\Gamma)$, and $(\# 2)$ from the fact that $(x, y) \mapsto$ $(y / x, x \backslash y)$ is a bijection, by definition of biracks. Step (\#3) follows from [4.7]

That $\mathcal{R R}^{-1}=1_{\mathbb{C}(\Gamma)} \otimes 1_{\mathbb{C}(\Gamma)}$ is proven analogously. 
Now note:

$$
\begin{aligned}
& \mathcal{R}_{12} \mathcal{R}_{13} \mathcal{R}_{23}=\sum_{x, y, a, z, u, v \in \Gamma_{0}}\left((x \stackrel{L(x, y)}{\longrightarrow} x \backslash y) \otimes(y \stackrel{R(x, y)}{\longrightarrow} y / x) \otimes 1_{\mathbb{C}(\Gamma)}\right) \\
& \left((a \stackrel{L(a, z)}{\longrightarrow} a \backslash z) \otimes 1_{\mathbb{C}(\Gamma)} \otimes(z \stackrel{R(a, z)}{\longrightarrow} z / a)\right) \\
& \left(1_{\mathbb{C}(\Gamma)} \otimes(u \stackrel{L(u, v)}{\longrightarrow} u \backslash v) \otimes(v \stackrel{R(u, v)}{\longrightarrow} v / u)\right) \\
& =\sum_{x, y, a, z, u, v \in \Gamma_{0}}((x \stackrel{L(x, y)}{\longrightarrow} x \backslash y)(a \stackrel{L(a, z)}{\longrightarrow} a \backslash z)) \otimes((y \stackrel{R(x, y)}{\longrightarrow} y / x)(u \stackrel{L(u, v)}{\longrightarrow} u \backslash v)) \\
& \otimes((z \stackrel{R(a, z)}{\longrightarrow} z / a)(v \stackrel{R(u, v)}{\longrightarrow} v / u)) \\
& \stackrel{(\# 1)}{=} \quad \sum_{x, y, a, z, u, v \in \Gamma_{0}} \quad((x \stackrel{L(x, y)}{\longrightarrow} x \backslash y)(a \stackrel{L(a, z)}{\longrightarrow} a \backslash z)) \otimes((y \stackrel{R(x, y)}{\longrightarrow} y / x)(u \stackrel{L(u, v)}{\longrightarrow} u \backslash v)) \\
& \begin{array}{c}
x, y, a, z, u, v \in \Gamma_{0} \\
a=x \backslash y \wedge u=y / x \wedge v=z / a
\end{array} \quad \otimes((z \stackrel{R(a, z)}{\longrightarrow} z / a)(v \stackrel{R(u, v)}{\longrightarrow} v / u)) \\
& \stackrel{(\# 2)}{=} \sum_{x, y, z \in \Gamma}(x \stackrel{L(x, y) \star L(x \backslash y, z)}{\longrightarrow}(x \backslash y) \backslash z) \otimes(y \stackrel{R(x, y) \star L(y / x, z /(x \backslash y))}{\longrightarrow}(y / x) \backslash(z /(x \backslash y))) \\
& \otimes(z \stackrel{R(x \backslash y, z) \star R(y / x, z /(x \backslash y))}{\longrightarrow}(z /(x \backslash y)) /(y / x)) \\
& \stackrel{(\# 3)}{=} \sum_{x, y, z \in \Gamma}(x \stackrel{L(x, z / y) \star L(x \backslash(z / y), y \backslash z)}{\longrightarrow}(x \backslash(z / y)) \backslash(y \backslash z)) \otimes(y \stackrel{L(y, z) \star R(x \backslash(z / y), y \backslash z)}{\longrightarrow}(y \backslash z) /(x \backslash(z / y))) \\
& \otimes(z \stackrel{R(y, z) \star R(x, z / y)}{\longrightarrow}(z / y) / x) \\
& =\mathcal{R}_{23} \mathcal{R}_{13} \mathcal{R}_{12} .
\end{aligned}
$$

Here (\#1) and (\#2) follow from (42). On the other hand (\#3) follows from (54), (61) and (62).

That $\mathcal{R}_{13} \mathcal{R}_{23}=\mathcal{R}_{23} \mathcal{R}_{13}$ if the bikoid is welded follows analogously, by using (53), (55), (63) and (64).

We can hence can define representations of the virtual braid group $\mathrm{VB}_{n}$ derived from a bikoid $\left(\Gamma, X^{+}\right)$. The following is one of our main results.

Theorem 38. Let $\left(\Gamma, X^{+}\right)$be a bikoid. Let $V$ be a right-representation of the groupoid algebra $\mathbb{C}(\Gamma)$; 3 3.2. Let $n \in \mathbb{Z}^{+}$. We have a representation $\triangleleft^{*}$ of $\mathrm{VB}_{n}$ on $V^{n \otimes}$, which on the generators of $\mathrm{VB}_{n}$ has the form:

$$
\begin{aligned}
\left(v_{1} \otimes \ldots \otimes v_{a-1}\right. & \left.\otimes v_{a} \otimes v_{a+1} \otimes v_{a+2} \otimes \ldots \otimes v_{n}\right) \triangleleft^{*} V_{a}[n]=v_{1} \otimes \ldots \otimes v_{a-1} \otimes v_{a+1} \otimes v_{a} \otimes v_{a+2} \otimes \ldots \otimes v_{n}, \\
\left(v_{1} \otimes \ldots \otimes v_{a-1} \otimes v_{a}\right. & \left.\otimes v_{a+1} \otimes v_{a+2} \otimes \ldots \otimes v_{n}\right) \triangleleft^{*} S_{a}^{+}[n] \\
= & \sum_{x, y \in \Gamma_{0}} v_{1} \otimes \ldots \otimes v_{a-1} \otimes v_{a+1} \cdot(y \stackrel{R(x, y)}{\longrightarrow} y / x) \otimes v_{a} .(x \stackrel{L(x, y)}{\longrightarrow} x \backslash y) \otimes v_{a+2} \otimes \ldots \otimes v_{n} .
\end{aligned}
$$

Moreover, $\triangleleft^{*}$ descends to a representation of $\mathrm{WB}_{n}$ on $V^{n \otimes}$ if $\left(\Gamma, X^{+}\right)$is welded.

These are unitary representations if $V$ is a unitary representation of the groupoid algebra; see Def. 6 .

[5.1] If $V$ is the object-regular representation (see Example 8) of $\mathbb{C}(\Gamma)$, then $\triangleleft^{*}$ coincides with $\triangleleft$ in 4.4, [5.2] If $V$ is the right-regular representation (see Example 7) of $\mathbb{C}(\Gamma)$, then $\triangleleft^{*}$ coincides with $\triangleleft^{*}$ in [4.9],

Proof. (We are using the definition in [3.12] for $\mathrm{WB}_{n}$ ). First of all note that $(-) \triangleleft^{*} S_{a}^{+}[n]$ and $(-) \triangleleft^{*} V_{a}[n]$ each are invertible maps $V^{n \otimes} \rightarrow V^{n \otimes}$. In order to prove $\triangleleft^{*}$ is a representation, we must check that the relations in Def. 11 for the virtual braid group, not involving the $S_{a}^{-}[n]$, hold. The Reidemeister III move follows from (73), whereas Virtual and Mixed Reidemeister III moves, as well as Locality, follow trivially. If $\left(\Gamma, X^{+}\right)$is welded, then (74) holds, from which it follows the Welded Reidemeister III move.

Let us now prove that the representation of $\mathrm{VB}_{n}$ is unitary if $V$ is unitary; see Def. 6. It suffices proving unitarity for $S_{a}^{+}[n]$. This follows easily from the following calculation, which encodes unitarity for $n=2$. If 
$u, v, u^{\prime}, v^{\prime} \in V$, we have:

$$
\begin{aligned}
& \left\langle\sum_{x, y \in \Gamma_{0}} u^{\prime} \cdot(y \stackrel{R(x, y)}{\longrightarrow} y / x) \otimes u \cdot(x \stackrel{L(x, y)}{\longrightarrow} x \backslash y), \sum_{z, w \in \Gamma_{0}} v^{\prime} \cdot(w \stackrel{R(z, w)}{\longrightarrow} w / z) \otimes v \cdot(z \stackrel{L(z, w)}{\longrightarrow} z \backslash w)\right\rangle \\
& \stackrel{(\# 1)}{=} \sum_{z, w \in \Gamma_{0}} \sum_{x, y \in \Gamma_{0}}\left\langle u^{\prime} \otimes u, v^{\prime} \cdot(w \stackrel{R(z, w)}{\longrightarrow} w / z) \star(y / x \stackrel{\overline{R(x, y)}}{\longrightarrow} y) \otimes v \cdot(z \stackrel{L(z, w)}{\longrightarrow} z \backslash w) \star\left(x \backslash y \stackrel{\frac{L(x, y)}{\longrightarrow}}{\longrightarrow} x\right)\right\rangle \\
& \stackrel{(\# 2)}{=}\left\langle u^{\prime} \otimes u, v^{\prime} \otimes v\right\rangle .
\end{aligned}
$$

In $(\# 1)$ we have used Def. [6 and the notation of [4.6] and [4.7] Step $(\# 2)$ follows from $($ in $\mathbb{C}(\Gamma) \otimes \mathbb{C}(\Gamma))$ :

$$
\begin{aligned}
& \sum_{z, w \in \Gamma_{0}} \sum_{x, y \in \Gamma_{0}}(w \stackrel{R(z, w)}{\longrightarrow} w / z) \star(y / x \stackrel{\overline{R(x, y)}}{\longrightarrow} y) \otimes(z \stackrel{L(z, w)}{\longrightarrow} z \backslash w) \star(x \backslash y \stackrel{\overline{L(x, y)}}{\longrightarrow} x) \\
& \stackrel{(\# 1)}{=} \sum_{z, w \in \Gamma_{0}} \sum_{\begin{array}{c}
x, y \in \Gamma_{0} \\
w / z=y / x \\
z \backslash w=x \backslash y
\end{array}}(w \stackrel{R(z, w)}{\longrightarrow} w / z) \star(y / x \stackrel{\overline{R(x, y)}}{\longrightarrow} y) \otimes(z \stackrel{L(z, w)}{\longrightarrow} z \backslash w) \star(x \backslash y \stackrel{\overline{L(x, y)}}{\longrightarrow} x) \\
& \stackrel{(\# 2)}{=} \sum_{z, w \in \Gamma_{0}}(w \stackrel{R(z, w)}{\longrightarrow} w / z) \star(w / z \stackrel{\overline{R(z, w)}}{\longrightarrow} w) \otimes(z \stackrel{L(z, w)}{\longrightarrow} z \backslash w) \star(z \backslash w \stackrel{\overline{L(z, w)}}{\longrightarrow} z) \\
& \stackrel{(\# 3)}{=} \sum_{z, w \Gamma_{0}}\left(w \stackrel{\mathrm{id}_{w}}{\longrightarrow} w\right) \otimes\left(z \stackrel{\mathrm{id}_{z}}{\longrightarrow} z\right)=1_{\mathbb{C}(\Gamma)} \otimes 1_{\mathbb{C}(\Gamma)} .
\end{aligned}
$$

Note that step (\#1) follows from (42). Step (\#2) follows from the fact that the map $(z, w) \mapsto(w / z, z \backslash w)$ is bijective, by Def. [19]. Step (\#3) follows from the description in [4.6] and [4.7] This finishes the proof.

The representation in Thm. 38 generalises. Pick different representations $V_{1}, \ldots, V_{n}$ of $\mathbb{C}(\Gamma)$, and consider $(-) \triangleleft^{*} S_{a}^{+}[n]$ and $(-) \triangleleft^{*} V_{a}[n]$ in (75) and (76) to act on $v_{1} \otimes \ldots \otimes v_{n} \in V_{1} \otimes \ldots \otimes V_{n}$. In general, this does not define a representation of $\mathrm{VB}_{n}$, since $(-) \triangleleft^{*} S_{a}^{+}[n]$ and $(-) \triangleleft^{*} V_{a}[n]$ each send $V_{1} \otimes \ldots \otimes V_{a} \otimes V_{a+1} \otimes \ldots \otimes V_{n}$ to $V_{1} \otimes \ldots \otimes V_{a+1} \otimes V_{a} \otimes \ldots \otimes V_{n}$, but it still satisfies the $\mathrm{VB}_{n}$ relations. We give details in the next subsection.

\subsection{Representations of the categories $\Gamma-\mathrm{WB}_{n}$ and $\mathcal{U} \Gamma-\mathrm{WB}_{n}$ from bikoids}

The results in this section are stated for W-bikoids and representations of $\mathrm{WB}_{n}$; see Def. 11 and Def. 25. All results apply, mutatis mutandis, to bikoids and the virtual braid group $\mathrm{VB}_{n}$. We work over $\mathbb{C}$.

Throughout the subsection, we fix $n \in \mathbb{Z}^{+}$. Given a pair of vector spaces $U$ and $V$, we put $L(U, V)$ to denote the vector space of linear maps $U \rightarrow V$. Given vector spaces $U_{1}, \ldots, U_{n}, V_{1}, \ldots, V_{n}$, linear maps $g_{1}: U_{1} \rightarrow V_{1}, \ldots, g_{n}: U_{n} \rightarrow V_{n}$, and $f \in \Sigma_{n}$, we put:

$$
f \triangleright\left(U_{1} \otimes \ldots \otimes U_{n} \stackrel{g_{1} \otimes \ldots \otimes g_{n}}{\longrightarrow} V_{1} \otimes \ldots \otimes V_{n}\right)=\left(U_{f(1)} \otimes \ldots \otimes U_{f(n)} \stackrel{g_{f(1)} \otimes \ldots \otimes g_{f(n)}}{\longrightarrow} V_{f(1)} \otimes \ldots \otimes V_{f(n)}\right) .
$$

This yields a linear map $f \triangleright(-)$, from $L\left(\otimes_{i=1}^{n} U_{i}, \otimes_{i=1}^{n} V_{i}\right)$ to $L\left(\otimes_{i=1}^{n} U_{f(i)}, \otimes_{i=1}^{n} V_{f(i)}\right)$.

Definition 39 (Categories $\mathcal{V}^{n}$ and $\mathcal{U} \mathcal{V}^{n}$ ). We define the following category $\mathcal{V}^{n}$. Objects are given by sequences $\underline{U}=\left(U_{1}, \ldots, U_{n}\right)$ of vector spaces. Given objects $\underline{U}$ and $\underline{V}=\left(V_{1}, \ldots, V_{n}\right)$, the morphisms $\underline{U} \rightarrow \underline{V}$ are given by linear maps $F: \otimes_{i=1}^{n} U_{i} \rightarrow \otimes_{i=1}^{n} V_{i}$. Given objects $\underline{U}, \underline{\underline{V}}, \underline{\underline{W}}$, as well as morphisms $F: \underline{\underline{U}} \rightarrow \underline{\underline{V}}$ and $G: \underline{V} \rightarrow \underline{W}$, their composition in $\mathcal{V}^{n}$ is given by the following composition of linear maps (see [3.2]):

$$
(\underline{U} \stackrel{F}{\rightarrow} \underline{V} \stackrel{G}{\rightarrow} \underline{W})=(\underline{U} \stackrel{G \circ F}{\rightarrow} \underline{W}) .
$$

The category $\mathcal{U} \mathcal{V}^{n}$ is defined analogously. Objects of $\mathcal{U} \mathcal{V}^{n}$ are given by sequences $\underline{U}=\left(U_{1}, \ldots, U_{n}\right)$ of vector spaces, provided with inner products, and the linear maps $F: \otimes_{i=1}^{n} U_{i} \rightarrow \otimes_{i=1}^{n} V_{i}$ are required to be unitary.

The symmetric group $\Sigma_{n}$ left-acts in the categories $\mathcal{V}^{n}$ and $\mathcal{U} \mathcal{V}^{n}$ by functors, by means of Equation (77). 
Definition 40 (Categories $\Gamma-\mathrm{WB}_{n}$ and $\left.\mathcal{U} \Gamma-\mathrm{WB}_{n}\right)$. Let $\Gamma$ be a groupoid. We have categories $\Gamma-\mathrm{WB}_{n}$ and $\mathcal{U} \Gamma-\mathrm{WB}_{n}$. Objects of $\Gamma-\mathrm{WB}_{n}$ are given by sequences $\underline{R}=\left(R_{1}, \ldots, R_{n}\right)$ of representations of $\mathbb{C}(\Gamma)$; cf. Given objects $\underline{R}$ and $\underline{S}$, morphisms $\underline{R} \stackrel{B}{\rightarrow} \underline{S}$ are given by welded braids $B \in \mathrm{WB}_{n}$ such that $U_{B}^{-1} \triangleright \underline{R}=\underline{S}$, where $U_{B}$ is the underlying permutation of $B$; see [3.14]. The composition in $\Gamma-\mathrm{WB}_{n}$ has the form below:

$$
\left(\underline{R} \stackrel{B}{\rightarrow} U_{B}^{-1} \triangleright \underline{R} \stackrel{B^{\prime}}{\longrightarrow} U_{B^{\prime}}^{-1} \triangleright U_{B}^{-1} \triangleright \underline{R}\right)=\left(\underline{R} \stackrel{B B^{\prime}}{\longrightarrow} U_{B^{\prime}}^{-1} \triangleright U_{B}^{-1} \triangleright \underline{R}\right)=\left(\underline{R} \stackrel{B B^{\prime}}{\longrightarrow} U_{B B^{\prime}}^{-1} \triangleright \underline{R}\right) .
$$

The category $\mathcal{U} \Gamma-\mathrm{WB}_{n}$ is similarly defined, the only difference being that the $R_{1}, \ldots, R_{n}$ are required to be unitary representations of $\mathbb{C}(\Gamma)$.

We can see morphisms of $\Gamma-\mathrm{WB}_{n}$ as welded braids, whose strands are coloured with representation of $\mathbb{C}(\Gamma)$.

Theorem 41. Let $\left(\Gamma, X^{+}\right)$be a W-bikoid. We have a functor $\mathcal{F}: \Gamma-\mathrm{WB}_{n} \rightarrow \mathcal{V}^{n}$. The functor $\mathcal{F}$ sends an $n$-tuple of representations $\underline{R}=\left(R_{1}, \ldots, R_{n}\right)$ of $\mathbb{C}(\Gamma)$ to $U(\underline{R})=\left(U\left(R_{1}\right), \ldots, U\left(R_{n}\right)\right)$, where $U\left(R_{i}\right)$ is the underlying vector space of $R_{i}$. On morphisms, $\mathcal{F}$ is uniquely specified by its value on the morphisms of the form $\underline{R} \stackrel{B}{\rightarrow} U_{B}^{-1} \triangleright \underline{R}$, where $B$ is a generator of the monoid $\mathrm{WB}_{n}$, as in Equation (43). In these particular cases, $\mathcal{F}$ has the form in shown in Equations (78), (79) and (80), below:

$$
\begin{gathered}
\mathcal{F}\left(\left(U_{1}, \ldots, U_{a}, U_{a+1}, \ldots, U_{n}\right) \stackrel{S_{a}^{+}[n]}{\longrightarrow}\left(U_{1}, \ldots, U_{a+1}, U_{a}, \ldots, U_{n}\right)\right)\left(v_{1} \otimes \ldots \otimes v_{a} \otimes v_{a+1} \otimes \ldots \otimes v_{n}\right) \\
=\sum_{x, y \in \Gamma_{0}} v_{1} \otimes \ldots \otimes v_{a-1} \otimes v_{a+1} \cdot(y \stackrel{R(x, y)}{\longrightarrow} y / x) \otimes v_{a} \cdot(x \stackrel{L(x, y)}{\longrightarrow} x \backslash y) \otimes v_{a+2} \otimes \ldots \otimes v_{n}, \\
\mathcal{F}\left(\left(U_{1}, \ldots, U_{a}, U_{a+1}, \ldots, U_{n}\right) \stackrel{S_{a}^{-}[n]}{\longrightarrow}\left(U_{1}, \ldots, U_{a+1}, U_{a}, \ldots, U_{n}\right)\right)\left(v_{1} \otimes \ldots \otimes v_{a} \otimes v_{a+1} \otimes \ldots \otimes v_{n}\right) \\
=\sum_{x, y \in \Gamma_{0}} v_{1} \otimes \ldots \otimes v_{a-1} \otimes v_{a+1} \cdot(x \backslash y \stackrel{\overline{L(x, y)}}{\longrightarrow} x) \otimes v_{a} \cdot(y / x \stackrel{\overline{R(x, y)}}{\longrightarrow} y) \otimes v_{a+2} \otimes \ldots \otimes v_{n}, \\
\mathcal{F}\left(\left(U_{1}, \ldots, U_{a}, U_{a+1}, \ldots, U_{n}\right) \stackrel{V_{a}[n]}{\longrightarrow}\left(U_{1}, \ldots, U_{a+1}, U_{a}, \ldots, U_{n}\right)\right)\left(v_{1} \otimes \ldots \otimes v_{a} \otimes v_{a+1} \otimes \ldots \otimes v_{n}\right) \\
=v_{1} \otimes \ldots \otimes v_{a-1} \otimes v_{a+1} \otimes v_{a} \otimes v_{a+2} \otimes \ldots \otimes v_{n} .
\end{gathered}
$$

Equations (78) -(80) also gives a functor $\mathcal{U F}: \mathcal{U} \Gamma-\mathrm{WB}_{n} \rightarrow \mathcal{U} \mathcal{V}^{n}$, sending $\underline{R}=\left(R_{1}, \ldots, R_{n}\right)$ to $U(\underline{R})=$ $\left(U\left(R_{1}\right), \ldots, U\left(R_{n}\right)\right)$, where $U\left(R_{i}\right)$ is the underlying inner-product space of the unitary representation $R_{i}$.

Proof. Follows from the calculations in the proof of Thm. 38. We can also combine [3.15] and Lem. 37.

\section{Bikoids from crossed modules}

We already have, from $\$ 5.1$ and $\$ 5.2$. machines for constructing representations of the welded braid group $\mathrm{WB}_{n}$, hence 3.4 of the loop braid group $\mathrm{LB}_{n}$, from W-bikoids. The task now is to manufacture W-bikoids from crossed modules. These will be our main examples of W-bikoids. They are related to Aharonov-Bohm like effects [3, 5, 42, inherent to moving loop-particles in topological higher gauge theory in $D^{3}$, see $\$ 2.3$.

Let the group $G$ have a left-action $(g, e) \in G \times E \mapsto g \triangleright e \in E$ on the group $E$ by automorphisms. In order to not surcharge our notation with brackets, let us convention that actions have priority over group multiplication, meaning for instance that we put $g \triangleright a g \triangleright b c$ for $(g \triangleright a)(g \triangleright b) c$, where $a, b, c \in E$ and $g, h \in G$.

The following simple observation will be useful later on in 86.2 .3 .

[6.1] Let $\Gamma=\left(\Gamma_{1}, \Gamma_{0}, \sigma, \tau, \iota, \star\right)$ and $\Gamma^{\prime}=\left(\Gamma_{1}^{\prime}, \Gamma_{0}^{\prime}, \sigma^{\prime}, \tau^{\prime}, \iota^{\prime}, \star^{\prime}\right)$ be groupoids. Let us be given a groupoid inclusion $g: \Gamma \rightarrow \Gamma^{\prime}=\left(g_{0}: \Gamma_{0} \rightarrow \Gamma_{0}^{\prime}, g_{1}: \Gamma_{1} \rightarrow \Gamma_{1}^{\prime}\right)$. Hence $g_{0}$ and $g_{1}$ are injective and preserve all structure 
maps in $\Gamma$ and $\Gamma^{\prime}$. Suppose in addition that $g_{0}$ is surjective. If we have a bikoid (or W-bikoid) $X_{\Gamma}^{+}=X^{+}$on $\Gamma$ then $X_{\Gamma}^{+}$can be transported to a bikoid (or W-bikoid) $X_{\Gamma^{\prime}}^{+}$in $\Gamma^{\prime}$, in the obvious way. Explicitly we put:

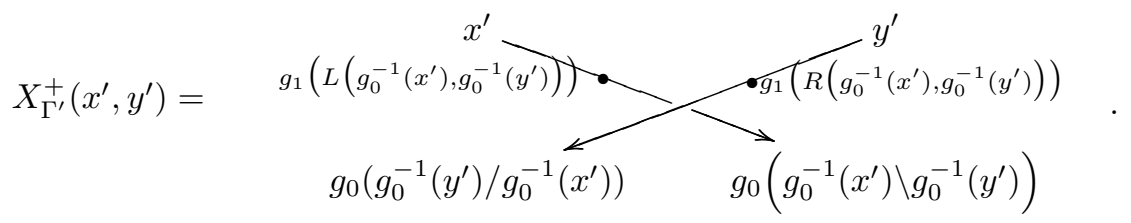

\subsection{Crossed modules $\mathcal{G}$}

Definition 42 (Crossed module). Let $E$ and $G$ be groups. A crossed module of groups $\mathcal{G}=(\partial: E \rightarrow G, \triangleright)$ (see [60, 71, 73, 74]) is a group map $\partial: E \rightarrow G$, and a left action of $G$ on $E$ by automorphisms, such that the relations below, called Peiffer relations hold for each $g \in G$ and $e, e^{\prime} \in E$ :

$$
\text { 1st Peiffer relation: } \partial(g \triangleright e)=g \partial(e) g^{-1}, \quad \text { 2nd Peiffer relation: } \partial(e) \triangleright e^{\prime}=e e^{\prime} e^{-1} \text {. }
$$

Lemma 43. Let $\mathcal{G}=(\partial: E \rightarrow G, \triangleright)$ be a crossed module. a) The group $A=\operatorname{ker}(\partial) \subset E$ is central in $E$, and in particular $A$ is abelian. b) The group $A$ is closed under the action of $G$, meaning that if $K \in A$ and $g \in G$, then $g \triangleright K \in A$. c) Given any $g, h \in G, e \in E$, and $f \in A$ it holds that: $(g \partial(e) h) \triangleright f=(g h) \triangleright f$. d) The action of $G$ on $A$ descends to an action of $\operatorname{coker}(\partial)$ on $A$.

Proof. Assertions a), c), d) follow from the 2nd Peiffer relation, and b) from the 1st Peiffer relation.

[6.2] A crossed module $\mathcal{G}$ hence gives rise to two abelian gr-groups: $(G, A)$ and (coker $(\partial), A)$; see Def. 30 .

[6.3] Conversely, if $(G, A, \triangleright)$ is an abelian $g r$-group, then we have a crossed module $(A \stackrel{a \mapsto 1}{\longrightarrow} G, \triangleright)$. Looking at the abelian $g r$-groups in Examples 31 and 34, this gives many examples of crossed modules of finite groups. [6.4] Let $G$ be a group and $\operatorname{Aut}(G)$ be its group of automorphisms, acting on $G$ in the obvious way. Let $g \in G \mapsto \operatorname{Ad}_{g} \in \operatorname{Aut}(G)$ be such that $\operatorname{Ad}_{g}(x)=g x g^{-1}$. This defines a crossed module (Ad: $\left.G \rightarrow \operatorname{Aut}(G), \triangleright\right)$.

\subsection{Groupoids $\operatorname{TRANS}\left(T_{R}^{2}(\mathcal{G})\right)$ and $\operatorname{TRANS}\left(S^{2}(\mathcal{G})\right)$, and the associated W-bikoids}

Throughout this subsection, we fix a crossed module $\mathcal{G}=(\partial: E \rightarrow G, \triangleright)$, and an element $R \in E$; see [2.18] We will explicitly construct the W-bikoids $X_{R}^{+}$and $X_{g r^{*}}^{+}$of Equations (39) in (41), appearing at the end of the physics motivation section $\$ 2.3$. We firstly construct $X_{g r^{*}}^{+}$, and them obtain $X_{R}^{+}$by transporting $X_{g r^{*}}^{+}$ along a groupoid isomorphism; see [6.1] The ideas are simple, but require several algebraic preliminaries. A slightly depleted version of the W-bikoids $X_{g r^{*}}^{+}$and $X_{R}^{+}$is the W-bikoid $X_{g r}^{+}$in Equation (69).

Our convention for commutators in a group $G$ is $[p, q]=p q p^{-1} q^{-1}$, where $p, q \in G$.

\subsubsection{The groups $T_{R}^{2}(\mathcal{G})$ and $S^{2}(\mathcal{G})$}

Definition 44. Fix an $R \in E$. We let $T_{R}^{2}(\mathcal{G})=\{(g, \partial(R), e) \in G \times G \times E \mid \partial(e)=[\partial(R), g]\}$.

Explicit calculations swiftly prove the following lemma. (Cf. 2.22] and the second equation of (24).)

Lemma 45. The set $T_{R}^{2}(\mathcal{G})$ is a group with the operation $(g, \partial(R), e)\left(g^{\prime}, \partial(R), e^{\prime}\right)=\left(g g^{\prime}, \partial(R)\right.$, e $\left.g \triangleright e^{\prime}\right)$. Hence inverses in $T_{R}^{2}(\mathcal{G})$ take the form $(g, \partial(R), e)^{-1}=\left(g^{-1}, \partial(R), g^{-1} \triangleright e^{-1}\right)$. The unit is $\left(1_{G}, \partial(R), 1_{E}\right)$.

Proof. Associativity is immediate. The most important bit is to show that $\partial\left(e g \triangleright e^{\prime}\right)=\left[\partial(R), g g^{\prime}\right]$. Note:

$$
\partial\left(e g^{-1} \triangleright e^{\prime}\right)=\partial(e) g^{-1} \partial\left(e^{\prime}\right) g=[\partial(R), g] g\left[\partial(R), g^{\prime}\right] g^{-1}=\left[\partial(R), g g^{\prime}\right],
$$

where we have used the 1st Peiffer relation in Def. 42

Definition 46. (Recall the conventions in 4.10]) Let $S^{2}(\mathcal{G})=G \ltimes_{\triangleright} A$. Here $A=\operatorname{ker}(\partial)$, which is closed under the action of $G$ on $E$; see Lem. 43 . Hence the product in $S^{2}(\mathcal{G})$ is $(g, K)(h, L)=(g h, K g \triangleright L)$.

[6.5] We have a group isomorphism $\phi_{1_{E}}:(g, J) \in S^{2}(\mathcal{G}) \mapsto\left(g, \partial\left(1_{E}\right), J\right) \in T_{1_{E}}^{2}(\mathcal{G})$. This generalises. 
Lemma 47. Let $R \in E$. We have a group isomorphism $\psi_{R}: T_{R}^{2}(\mathcal{G}) \rightarrow S^{2}(\mathcal{G})$. It is defined as:

$$
(g, \partial(R), e) \stackrel{\psi_{R}}{\longmapsto}\left(g, R^{-1} \text { e } g \triangleright R\right) .
$$

Its inverse is given by $\phi_{R}: S^{2}(\mathcal{G}) \rightarrow T_{R}^{2}(\mathcal{G})$, where $\phi_{R}(g, J)=\left(g, \partial(R), R J g \triangleright R^{-1}\right)$.

Proof. Let $(g, \partial(R), e) \in T_{R}^{2}(\mathcal{G})$. Then $\partial(e)=[\partial(R), g]$, from which it follows, by using $\partial(g \triangleright R)=g \partial(R) g^{-1}$, that $R^{-1} e g \triangleright R \in A=\operatorname{ker}(\partial)$. Thence $\left(g, R^{-1} e g \triangleright R\right) \in S^{2}(\mathcal{G})$. Analogously, if $J$ in $\operatorname{ker}(\partial)$, it holds that $\partial\left(R J g \triangleright R^{-1}\right)=[\partial(R), g]$. (We have used the 1st Peiffer rule in (81).) Hence $\phi_{R}(g, J) \in T_{R}^{2}(\mathcal{G})$, if $g \in G$.

Given $(g, \partial(R), e),\left(g^{\prime}, \partial(R), e^{\prime}\right) \in T_{R}^{2}(\mathcal{G})$, we have:

$$
\begin{aligned}
\psi_{R}\left((g, \partial(R), e)\left(g^{\prime}, \partial(R), e^{\prime}\right)\right) & =\psi_{R}\left(g g^{\prime}, \partial(R), \text { e } g \triangleright e^{\prime}\right)=\left(g g^{\prime}, R^{-1} \text { e } g \triangleright e^{\prime}\left(g g^{\prime}\right) \triangleright R\right) \\
& =\left(g g^{\prime}, R^{-1} \text { e } g \triangleright R g \triangleright R^{-1} g \triangleright e^{\prime}\left(g g^{\prime}\right) \triangleright R\right)=\left(g, R^{-1} \text { e } g \triangleright R\right)\left(g^{\prime}, R^{-1} e^{\prime} g^{\prime} \triangleright R\right) \\
& =\psi_{R}(g, \partial(R), e) \psi_{R}\left(g^{\prime}, \partial(R), e^{\prime}\right) .
\end{aligned}
$$

To finalise, note that clearly $\psi_{R} \circ \phi_{R}=\operatorname{id}_{S^{2}(\mathcal{G})}$ and $\phi_{R} \circ \psi_{R}=\operatorname{id}_{T_{R}^{2}(\mathcal{G})}$.

[6.6] We have a homomorphism $\Theta: S^{2}(\mathcal{G}) \rightarrow S^{2}(\mathcal{G})$ defined as: $(g, J) \stackrel{\Theta}{\mapsto}\left(g, 1_{A}\right)$. Note $\Theta \circ \Theta=\Theta$. By combining with the previous lemma, or by a direct calculation, it follows that:

Lemma 48. We have a group homomorphism $\Theta_{R}: T_{R}^{2}(\mathcal{G}) \rightarrow T_{R}^{2}(\mathcal{G})$, which has the explicit form:

$$
(g, \partial(R), e) \in T_{R}^{2}(\mathcal{G}) \stackrel{\Theta_{R}}{\longmapsto}\left(g, \partial(R), R g \triangleright R^{-1}\right) \in T_{R}^{2}(\mathcal{G}) .
$$

Furthermore $\Theta_{R} \circ \Theta_{R}=\Theta_{R}$ and $\Theta_{R}=\phi_{R} \circ \Theta \circ \psi_{R}$.

[6.7] We will make strong use of the map of sets $\beta: S^{2}(\mathcal{G}) \rightarrow S^{2}(\mathcal{G})$, defined as $(g, K) \stackrel{\beta}{\mapsto}\left(1_{G}, K^{-1}\right)$. Note that $\beta(g, K)=\left(g, 1_{A}\right)(g, K)^{-1}$, in other words: $\beta(g, K)=\Theta(g, K)(g, K)^{-1}$, for each $(g, K) \in S^{2}(\mathcal{G})$.

Definition 49. Analogoulsy, if $(g, \partial(R), e) \in T_{R}^{2}(\mathcal{G})$, we put $\beta_{R}(g, \partial(R), e) \in T_{R}^{2}(\mathcal{G})$ to be:

$$
\begin{aligned}
\beta_{R}(g, \partial(R), e) & =\Theta_{R}(g, \partial(R), e)(g, \partial(R), e)^{-1} \\
& =\left(1_{G}, \partial(R), R g \triangleright R^{-1} e^{-1}\right) \in T_{R}^{2}(\mathcal{G}) .
\end{aligned}
$$

[6.8] By construction, or by a direct calculation, it follows that: $\beta_{R}=\phi_{R} \circ \beta \circ \psi_{R}$.

Lemma 50. We have left-actions $>$ of $E$ on $T_{R}^{2}(\mathcal{G})$ and on $S_{R}^{2}(\mathcal{G})$. They are such that:

$$
\begin{aligned}
a \gg(g, \partial(R), e) & =\left(\partial(a) g, \partial(R), R a R^{-1} \text { e } a^{-1}\right), \\
a \gg(g, K) & =(\partial(a) g, K) .
\end{aligned}
$$

Here $a \in E$. Morever $\psi_{R}: T_{R}^{2}(\mathcal{G}) \rightarrow S^{2}(\mathcal{G})$, hence also $\phi_{R}: S^{2}(\mathcal{G}) \rightarrow T_{R}^{2}(\mathcal{G})$, preserves $E$ actions.

(The action in Equation (84) was mentioned in [2.23] It arises from the gauge transformation rule for the 2-dimensional holonomy of a 2-connection (27).) These actions are, in general, not actions by automorphisms.

Proof. Firstly, if $(g, \partial(R), e) \in T_{R}^{2}(\mathcal{G})$, then an easy calculation shows that $\partial\left(R a R^{-1} e a^{-1}\right)=[\partial(R), \partial(a) g]$. Let $a, b \in E$. Clearly $a>b>(-)=(a b)>(-)$, both for $>$ in (84) and $>$ in (85). Finally note that:

$$
\begin{aligned}
\psi_{R}(a \gg(g, \partial(R), e)) & =\psi_{R}\left(\partial(a) g, \partial(R), R a R^{-1} \text { e } a^{-1}\right)=\left(\partial(a) g, R^{-1} R a R^{-1} \text { e } a^{-1}(\partial(a) g) \triangleright R\right) \\
& \stackrel{(\# 1)}{=}\left(\partial(a) g, R^{-1} R \text { a } R^{-1} \text { e } a^{-1} \text { a } g \triangleright R a^{-1}\right) \\
& =\left(\partial(a) g, a R^{-1} \text { e } g \triangleright R a^{-1}\right),
\end{aligned}
$$

where (\#1) follows from the 2nd Peiffer rule (see Def. 42). On the other hand:

$$
\begin{aligned}
a \gg \psi_{R}(g, \partial(R), e) & =a \gg\left(g, R^{-1} \text { e } g \triangleright R\right)=\left(\partial(a) g, R^{-1} \text { e } g \triangleright R\right) \\
& \stackrel{(\# 2)}{=}\left(\partial(a) g, a R^{-1} \text { e } g \triangleright R a^{-1}\right) .
\end{aligned}
$$

Here step (\#2) follows from the fact that $\operatorname{ker}(\partial)=A$ is central in $E$ (Lem. 43), since $R^{-1}$ e $g \triangleright R \in A$. 


\subsubsection{The action groupoids $\operatorname{TRANS}\left(S^{2}(\mathcal{G})\right)$ and $\operatorname{TRANS}\left(T_{R}^{2}(\mathcal{G})\right)$}

[6.9] Consider the actions $\triangleright^{\prime}$ of $T_{R}^{2}(\mathcal{G})$ and of $S^{2}(\mathcal{G})$ on $E$, by automorphisms, such that:

$$
\begin{aligned}
(g, \partial(R), e) \triangleright^{\prime} a & =g \triangleright a, \text { where } a \in E \text { and }(g, \partial(R), e) \in T_{R}^{2}(\mathcal{G}), \\
(g, K) \triangleright^{\prime} a & =g \triangleright a, \text { where } a \in E \text { and }(g, K) \in S^{2}(\mathcal{G}) .
\end{aligned}
$$

[6.10] Note that $\psi_{R}(g, \partial(R), e) \triangleright^{\prime} a=(g, \partial(R), e) \triangleright^{\prime} a$, where $a \in E$ and $(g, \partial(R), e) \in T_{R}^{2}(\mathcal{G})$.

[6.11] Let $>$ denote the left-actions of $T_{R}^{2}(\mathcal{G})$ and of $S^{2}(\mathcal{G})$ on themselves by conjugation.

Lemma 51. We have a left-action $>$ of the group $T_{R}^{2}(\mathcal{G}) \ltimes_{\triangleright^{\prime}} E$ (cf. [4.10]) on $T_{R}^{2}(\mathcal{G})$. It has the form:

$$
\begin{aligned}
(g, \partial(R), e, a) \gg(h, \partial(R), f) & =a \gg((g, \partial(R), e) \gg(h, \partial(R), f)) \\
& =\left(\partial(a) g h g^{-1}, R a R^{-1} \text { e } g \triangleright f\left(g h g^{-1}\right) \triangleright e^{-1} a^{-1}\right) .
\end{aligned}
$$

Proof. Cf. Lem. 50 and 4.11] We solely need to prove that, given $a \in E$ and $(g, \partial(R), e) \in T_{R}^{2}(\mathcal{G})$, then:

$$
a \gg((g, \partial(R), e) \gg(h, \partial(R), f))=(g, \partial(R), e) \gg\left(\left(g^{-1} \triangleright a\right) \gg(h, \partial(R), e)\right) .
$$

We have:

$$
\begin{aligned}
a \gg((g, \partial(R), e) \gg(h, \partial(R), f)) & =a \gg\left(g h g^{-1}, \partial(R), e g \triangleright f\left(g h g^{-1}\right) \triangleright e^{-1}\right) \\
& =\left(\partial(a) g h g^{-1}, \partial(R), R a R^{-1} \text { e } g \triangleright f\left(g h g^{-1}\right) \triangleright e^{-1} a^{-1}\right) .
\end{aligned}
$$

On the other hand:

$$
\begin{aligned}
& (g, \partial(R), e) \gg\left(\left(g^{-1} \triangleright a\right) \gg(h, \partial(R), f)\right)=(g, \partial(R), e) \gg\left(g^{-1} \partial(a) g h, R g^{-1} \triangleright a R^{-1} f g^{-1} \triangleright a^{-1}\right) \\
& =\left(\partial(a) g h g^{-1}, \text { e } g \triangleright R \text { a } g \triangleright R^{-1} g \triangleright f a^{-1}\left(\partial(a) g h g^{-1}\right) \triangleright e^{-1}\right) \\
& \stackrel{(\# 1)}{=}\left(\partial(a) g h g^{-1}, \text { e } g \triangleright R a g \triangleright R^{-1} g \triangleright f\left(g h g^{-1}\right) \triangleright e^{-1} a^{-1}\right) \\
& \stackrel{(\# 2)}{=}\left(\partial(a) g h g^{-1}, R a R^{-1} \text { e } g \triangleright f\left(g h g^{-1}\right) \triangleright e^{-1} a^{-1}\right) \text {. }
\end{aligned}
$$

Here (\#1) follows by the 2nd. Peiffer rule (81). Step (\#2) also follows from the 2nd Peiffer rule, since:

$$
\begin{aligned}
e g \triangleright R a g \triangleright R^{-1} & \stackrel{(\# 1)}{=} \partial(e) \triangleright\left(g \triangleright R a g \triangleright R^{-1}\right) e \stackrel{(\# 2)}{=}\left(\partial(R) g \partial(R)^{-1} g^{-1}\right) \triangleright\left(g \triangleright R a g \triangleright R^{-1}\right) e \\
& \stackrel{(\# 3)}{=}\left(\partial(R) g \partial\left(R^{-1}\right)\right) \triangleright\left(R g^{-1} \triangleright a R^{-1}\right) e \\
& \stackrel{(\# 4)}{=} R g \triangleright\left(R^{-1} R g^{-1} \triangleright a R^{-1} R\right) R^{-1} e=R a R^{-1} e .
\end{aligned}
$$

In (\#2) we used $\partial(e)=\partial(R) g \partial(R)^{-1} g^{-1}$. The 2nd Peiffer rule was used in (\#1) and (\#4).

Definition 52. Consider the group $S^{2}(\mathcal{G}) \ltimes_{\triangleright^{\prime}} E=G \ltimes_{\triangleright}(A \times E)$, where $\triangleright$ is the product action of $G$ on $A \times E$. By [6.10]] and Lem. 47, we can define a group isomorphism $\psi_{R}^{*}: T_{R}^{2}(\mathcal{G}) \ltimes_{\triangleright^{\prime}} E \rightarrow S^{2}(\mathcal{G}) \ltimes_{\triangleright^{\prime}} E$, as:

$$
\psi_{R}^{*}(g, \partial(R), e, a)=\left(\psi_{R}(g, \partial(R), e), a\right), \text { where }(g, \partial(R), e) \in T_{R}^{2}(\mathcal{G}) \text { and } a \in E .
$$

[6.12] The inverse $\phi_{R}^{*}: S^{2}(\mathcal{G}) \ltimes_{\triangleright^{\prime}} E \rightarrow T_{R}^{2}(\mathcal{G}) \ltimes_{\triangleright^{\prime}} E$ of $\psi_{R}^{*}$ is such that $\phi_{R}^{*}(g, K, a)=\left(\phi_{R}(g, K), a\right)$.

Lemma 53. We have a left-action $\gg$ of $S^{2}(\mathcal{G}) \ltimes_{\triangleright^{\prime}} E=G \ltimes_{\triangleright}(A \times E)$, on $S^{2}(\mathcal{G})$. It has the form:

$$
(g, J, a) \gg(h, K)=a \gg((g, J) \gg(h, K))=\left(\partial(a) g h g^{-1}, J g \triangleright K(g h g)^{-1} \triangleright J^{-1}\right) .
$$

Moreover, given $(g, J, a) \in S^{2}(\mathcal{G}) \ltimes_{\triangleright^{\prime}} E$ and $(h, K) \in S^{2}(E)$, it holds that:

$$
\phi_{R}((g, J, a)>(h, K))=\phi_{R}^{*}(g, J, a)>\phi_{R}(h, K) .
$$

Note that $>$ is not, in general, an action by automorphisms. 
Proof. That we have an action follows as in the proof of Lem. 51] or by using Lem. 51 in the particular case $R=1_{E}$, given [6.5], The other bits follows from concatenation of previous formulae. Here is a proof:

$$
\begin{aligned}
\phi_{R}((g, J, a) \triangleright(h, K)) & =\phi_{R}\left(\partial(a) g h g^{-1}, J g \triangleright K(g h g)^{-1} \triangleright J^{-1}\right) \\
& =\left(\partial(a) g h g^{-1}, \partial(R), R J g \triangleright K\left(g h g^{-1}\right) \triangleright J^{-1}\left(\partial(a) g h g^{-1}\right) \triangleright R^{-1}\right) \\
& =\left(\partial(a) g h g^{-1}, \partial(R), R J g \triangleright K\left(g h g^{-1}\right) \triangleright J^{-1} a\left(g h g^{-1}\right) \triangleright R^{-1} a^{-1}\right) .
\end{aligned}
$$

In the last step we used the 2nd Peiffer relation (see Def. 42). On the other hand:

$$
\begin{aligned}
\phi_{R}^{*}(g, J, a) \gg & \phi_{R}(h, K)=\left(g, \partial(R), R J g \triangleright R^{-1}, a\right) \gg\left(h, \partial(R), R K h \triangleright R^{-1}\right) \\
& =\left(\partial(a) g h g^{-1}, \partial(R), R a R^{-1} R J g \triangleright R^{-1} g \triangleright\left(R K h \triangleright R^{-1}\right)\left(g h g^{-1}\right) \triangleright\left(R J g \triangleright R^{-1}\right)^{-1} a^{-1}\right) \\
& \stackrel{(\# 1)}{=}\left(\partial(a) g h g^{-1}, \partial(R), R a J g \triangleright K\left(g h g^{-1}\right) \triangleright J^{-1}\left(g h g^{-1}\right) \triangleright R^{-1} a^{-1}\right) \\
& \stackrel{(\# 2)}{=}\left(\partial(a) g h g^{-1}, \partial(R), R J g \triangleright K\left(g h g^{-1}\right) \triangleright J^{-1} a\left(g h g^{-1}\right) \triangleright R^{-1} a^{-1}\right) .
\end{aligned}
$$

Here (\#1) follows by cancelling pairs of a group element product with its inverse. And (\#2) follows from the fact that $A$ is central in $E$ and $J, K \in A$; recall Lem. 43 .

Definition 54. Cf. Def. 2] $\operatorname{TRANS}\left(S^{2}(\mathcal{G})\right)$ is the action groupoid of the action $\gg$ of $S^{2}(\mathcal{G}) \ltimes_{\triangleright^{\prime}} E$ on $S^{2}(\mathcal{G})$.

Definition 55. We let $\operatorname{TRANS}\left(T_{R}^{2}(\mathcal{G})\right)$ be the action groupoid of the action $\gg$ of $T_{R}^{2}(\mathcal{G}) \ltimes_{\triangleright^{\prime}} E$ on $T_{R}^{2}(\mathcal{G})$.

Arrows of $\operatorname{TRANS}\left(S^{2}(\mathcal{G})\right)$ and of $\operatorname{TRANS}\left(T_{R}^{2}(\mathcal{G})\right)$ hence have the form below; see (86) and (87):

$$
((h, \partial(R), f) \stackrel{(g, \partial(R), e, a)}{\longrightarrow}(g, \partial(R), e, a)>(h, \partial(R), f)) \text { and }((h, K) \stackrel{(g, J, a)}{\longrightarrow}(g, J, a)>(h, K)) .
$$

Here $g, h \in G ; e, a, f \in E$ and $J, K \in A$. For an interpretation in terms of 2-fluxes of loop-particles see $\$ 2.3$

Lemma 56. We have an isomorphism $\Phi_{R}: \operatorname{TRANS}\left(S^{2}(\mathcal{G})\right) \rightarrow \operatorname{TRANS}\left(T_{R}^{2}(\mathcal{G})\right)$, of groupoids. On objects $\Phi_{R}$ is given by $\phi_{R}$, see Lem. 47. On morphisms $\Phi_{R}$ takes the form (see [6.12]):

$$
\Phi_{R}((h, K) \stackrel{(g, J, a)}{\longrightarrow}(g, J, a)>(h, K))=\left(\phi_{R}(h, K) \stackrel{\phi_{R}^{*}(g, J, a)}{\longrightarrow} \phi_{R}^{*}(g, J, a) \gg \phi_{R}(h, K)\right) .
$$

Proof. Follows by combining Lem. 47, Lem 53 and Def. 52 ,

\subsubsection{A W-bikoid structure on $\operatorname{TRANS}\left(S^{2}(\mathcal{G})\right)$}

Let $\mathcal{G}=(\partial: E \rightarrow G, \triangleright)$ be a crossed module. We put $\bar{g}$ to denote $g^{-1}$. Recall [87), 4.11] and Def. [54,

Theorem 57. We have a $W$-bikoid structure $X_{g r^{*}}^{+}$in the groupoid $\operatorname{TRANS}\left(S^{2}(\mathcal{G})\right)$, such that:

$$
X_{g r^{*}}^{+}((z, J),(w, K))=\underbrace{(z, J)}_{(\bar{w} \triangleright \bar{J})>(w, K)}, \text { where }\left\{\begin{array}{l}
\bar{w}=\left(w^{-1}, 1_{A}, 1_{E}\right) \\
\bar{w} \bar{J}=\left(1_{G}, w^{-1} \triangleright J^{-1}, 1_{E}\right)
\end{array}\right.
$$

(Note that $\bar{w} \triangleright \bar{J}=\left(\beta\left(\Theta(w, K)^{-1} \rightarrow(z, J)\right), 1_{E}\right)$; see [6.7], Equation (88) can be written as (recall [6.11]):

$$
X_{g r^{*}}^{+}((z, J),(w, K))=\underbrace{\left(\Theta(w, K)^{-1}, 1_{E}\right)}_{\beta\left(\Theta(w, K)^{-1}>(z, J)\right)>(w, K)}
$$


Explicitly, the underlying $W$-birack of $X_{g r^{*}}^{+}$is such that (where $z, w \in G$ and $K, L \in A=\operatorname{ker}(\partial)$ ):

$$
\begin{aligned}
& (z, J) \backslash(w, K)=\bar{w} \gg(z, J)=\left(w^{-1}, 1_{A}, 1_{E}\right) \gg(z, J)=\left(w^{-1} z w, w^{-1} \triangleright J\right), \\
& (w, K) /(z, J)=(\bar{w} \triangleright \bar{J}) \gg(w, K)=\left(1_{G}, w^{-1} \triangleright J^{-1}, 1_{E}\right) \gg(w, K)=\left(w, w^{-1} \triangleright J^{-1} K J\right) .
\end{aligned}
$$

And, as the diagram in (88) indicates, the holonomy morphisms in the $W$-bikoid $X_{g r^{*}}^{+}$in (88) are:

$$
\begin{aligned}
& L((z, J),(w, K))=\left((z, J) \stackrel{\left(w^{-1}, 1_{A}, 1_{E}\right)}{\longrightarrow}\left(w^{-1}, 1_{A}, 1_{E}\right)>(z, J)\right), \\
& R((z, J),(w, K))=\left((w, K) \stackrel{\left(1_{G}, w^{-1} \triangleright J^{-1}, 1_{E}\right)}{\longrightarrow}\left(1_{G}, w^{-1} \triangleright J^{-1}, 1_{E}\right)>(w, K)\right) .
\end{aligned}
$$

Remark 58. The form (89) for the bikoid $X_{g r^{*}}^{+}$is useful (see 86.2 .4 ) for transporting [6.1] $X_{g r^{*}}^{+}$in (88) to $\operatorname{TRANS}\left(T_{R}^{2}(\mathcal{G})\right)$. Equation (89) for $X_{g r^{*}}^{+}$also makes the interpretation of the associated representations $\$ 5$ of the loop braid group as observables in topological higher gauge theory in $D^{3}$ more transparent; see 2.3 ,

Remark 59. A more compact formulation of $X_{g r^{*}}^{+}$in (88), in additive notation, can be found in (41).

Proof. That (88) and (89) coincide follows from [6.6] and [6.7]

Let us prove that $X_{g r^{*}}^{+}$in (88) is a bikoid. We freely use Defs. 19 and 22. First of all note that the maps:

$$
\begin{aligned}
& f^{(w, K)}:(z, J) \in S^{2}(\mathcal{G}) \longmapsto(z, J) \backslash(w, K)=\left(w^{-1}, 1_{A}, 1_{E}\right) \gg(z, J)=\left(w^{-1} z w, w^{-1} \triangleright J\right) \in S^{2}(\mathcal{G}), \\
& f_{(z, J)}:(w, K) \in S^{2}(\mathcal{G}) \longmapsto(w, K) /(z, J)=\left(1_{G}, w^{-1} \triangleright J^{-1}, 1_{E}\right) \gg(w, K)=\left(w, w^{-1} \triangleright J^{-1} K J\right) \in S^{2}(\mathcal{G})
\end{aligned}
$$

each are bijections. Inverses are given by:

$$
\begin{aligned}
& \overline{f^{(w, K)}}:\left(z^{\prime}, J^{\prime}\right) \in S^{2}(\mathcal{G}) \longmapsto\left(w, 1_{A}, 1_{E}\right) \gg\left(z^{\prime}, J^{\prime}\right)=\left(w z^{\prime} w^{-1}, w \triangleright J^{\prime}\right) \in S^{2}(\mathcal{G}), \\
& \overline{f_{(z, J)}}:\left(w^{\prime}, K^{\prime}\right) \in S^{2}(\mathcal{G}) \longmapsto\left(1_{G}, w^{\prime-1} \triangleright J, 1_{E}\right) \gg\left(w^{\prime}, K^{\prime}\right)=\left(w^{\prime}, w^{\prime-1} \triangleright J K^{\prime} J^{-1}\right) \in S^{2}(\mathcal{G}) .
\end{aligned}
$$

On the other hand, the map below is also invertible:

$$
S:((z, J),(w, K)) \in S^{2}(\mathcal{G}) \times S^{2}(\mathcal{G}) \mapsto((w, K) /(z, J),(z, J) \backslash(w, K)) \in S^{2}(\mathcal{G}) \times S^{2}(\mathcal{G}),
$$

and its inverse is:

$\left(\left(w^{\prime}, K^{\prime}\right),\left(z^{\prime}, J^{\prime}\right)\right) \mapsto\left(\left(w^{\prime}, 1_{A}, 1_{E}\right) \gg\left(z^{\prime}, J^{\prime}\right),\left(1_{G}, J^{\prime}, 1_{E}\right) \gg\left(w^{\prime}, K^{\prime}\right)\right)=\left(\left(w^{\prime} z^{\prime} w^{\prime-1}, w^{\prime} \triangleright J^{\prime}\right),\left(w^{\prime}, J^{\prime} K^{\prime} w^{\prime} \triangleright J^{\prime-1}\right)\right)$.

That $((z, J),(w, K)) \in S^{2}(\mathcal{G}) \times S^{2}(\mathcal{G}) \mapsto((w, K) /(z, J),(z, J) \backslash(w, K)) \in S^{2}(\mathcal{G}) \times S^{2}(\mathcal{G})$ in Equation (90) is a birack, and that $X_{g r^{*}}^{+}$in (88) is a bikoid, follows from comparing the diagrams (91) and (92) in $\operatorname{TRANS}\left(T_{R}^{2}(\mathcal{G})\right)^{3} \rtimes \Sigma_{3}$, where $(z, J),(w, K)$ and $(t, L)$ are general elements of $S^{2}(\mathcal{G})$.

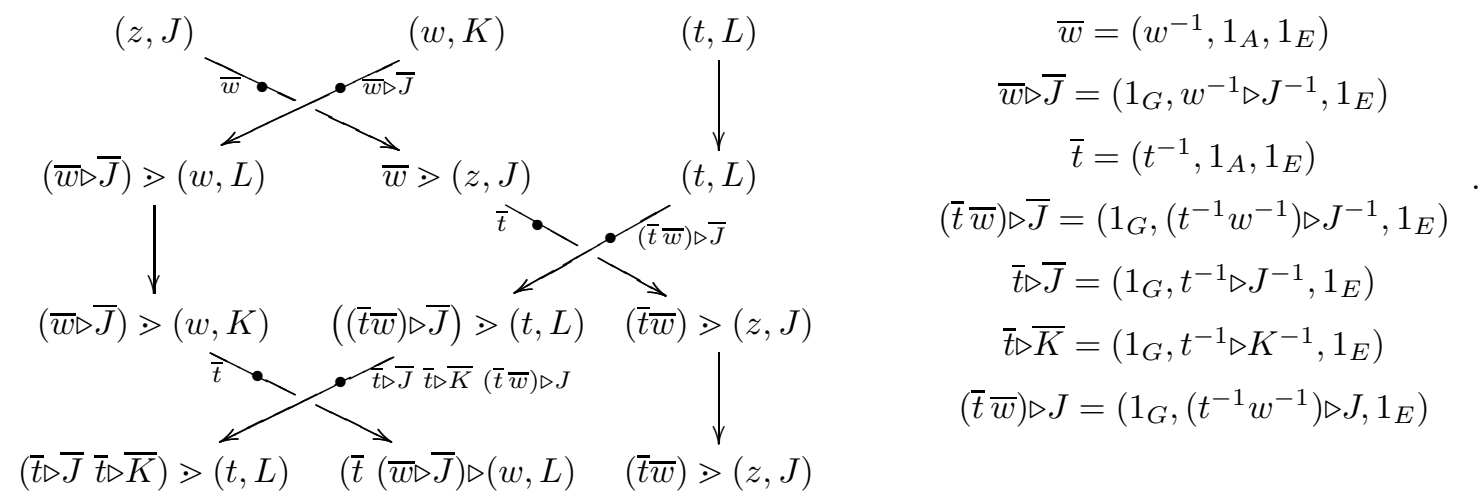


(Recall the convention in Def. 2 for the composition in $\operatorname{TRANS}\left(T_{R}^{2}(\mathcal{G})\right)$. Note:

$$
\begin{aligned}
\bar{w} \gg(z, J) & =\left(w^{-1}, 1_{A}, 1_{E}\right) \gg(z, J)=\left(w^{-1} z w, w^{-1} \triangleright J\right), \\
(\bar{w} \triangleright \bar{J}) \gg(w, K) & =\left(1_{G}, w^{-1} \triangleright J^{-1}, 1_{E}\right) \gg(w, K)=\left(w, w^{-1} \triangleright J^{-1} K J\right) \\
\left(1_{G}, t^{-1} \triangleright\left(w^{-1} \triangleright J^{-1} K J\right)^{-1}\right) & =\left(1_{G}, t^{-1} \triangleright J^{-1} t^{-1} \triangleright K^{-1}\left(t^{-1} w^{-1}\right) \triangleright J\right)=\bar{t} \triangleright \bar{J} \bar{t} \triangleright \bar{K}(\bar{t} \bar{w}) \triangleright J .
\end{aligned}
$$

And (for the other side of the Reidemeister III move):

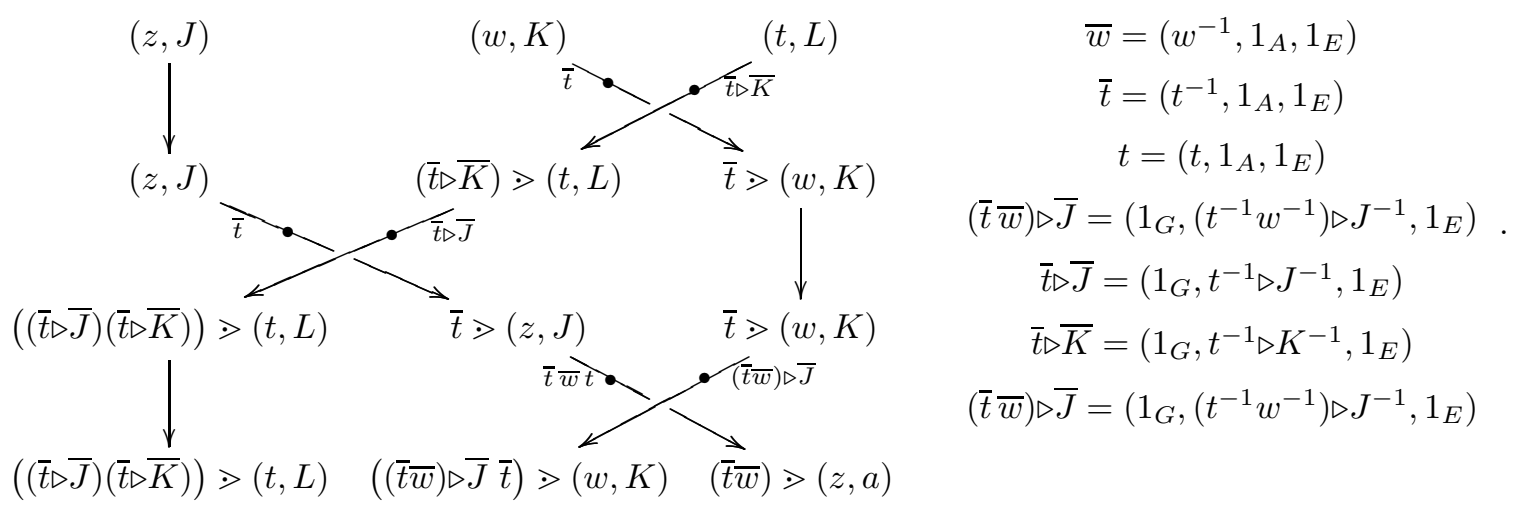

Note:

$$
\bar{t} \gg(w, K)=\left(t^{-1} w t, t^{-1} \triangleright K\right), \quad \bar{t} \gg(z, J)=\left(t^{-1} z t, t^{-1} \triangleright J\right) .
$$

We can easily see that the group elements in $S^{2}(\mathcal{G}) \ltimes_{\triangleright^{\prime}} E$ associated to each strand in the diagrams in (91) and (92) coincide. Namely in the first strand we have $\bar{t} \bar{w}=\bar{t} \bar{w} t \bar{t}$; in the second strand we have:

$$
\bar{t}(\bar{w} \triangleright \bar{J})=\left(t^{-1}, 1_{A}, 1_{E}\right)\left(1_{G}, w^{-1} \triangleright J^{-1}, 1_{E}\right)=\left(1_{G},\left(t^{-1} w^{-1}\right) \triangleright J^{-1}, 1_{E}\right)\left(t^{-1}, 1_{A}, 1_{E}\right)=((\bar{t} \bar{w}) \triangleright \bar{J}) \bar{t} .
$$

And in the third strand we have: $(\bar{t} \triangleright \bar{J} \bar{t} \triangleright \bar{K}(\bar{t} \bar{w}) \triangleright J)(\bar{t} \bar{w}) \triangleright \bar{J})=\bar{t} \triangleright \bar{J} \bar{t} \triangleright \bar{K}$.

Hence given any $(z, J),(w, K),(t, L) \in S^{2}(\mathcal{G})$, then diagrams (91) and (92) in $\operatorname{TRANS}\left(T_{R}^{2}(\mathcal{G})\right)^{3} \rtimes \Sigma_{3}$ coincide. Hence, by construction, so do the bottom lines of (91) and (92), thus it follows that $\left(S^{2}(\mathcal{G}), /, \backslash\right)$ in (90) is a birack. And then the equality of (91) and (92) mean exactly that $X_{g r^{*}}^{+}$in (88) is a bikoid.

That the bikoid is welded (Def. [25), follows in exactly the same way, by comparing the two diagrams below (93) and (94) in $\operatorname{TRANS}\left(S^{2}(\mathcal{G})\right)^{3} \rtimes \Sigma_{3}$, where $(z, J),(w, K),(t, L) \in S^{2}(\mathcal{G})$ :

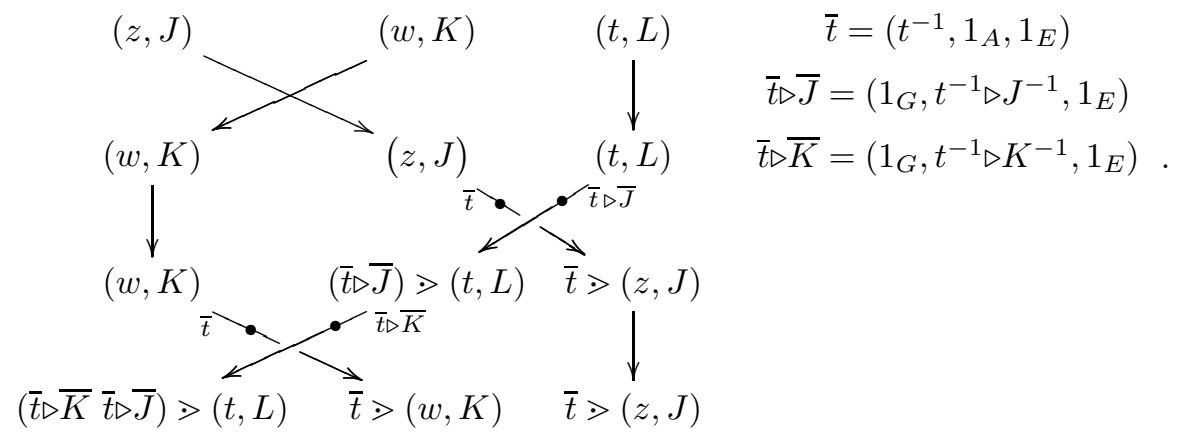

and:

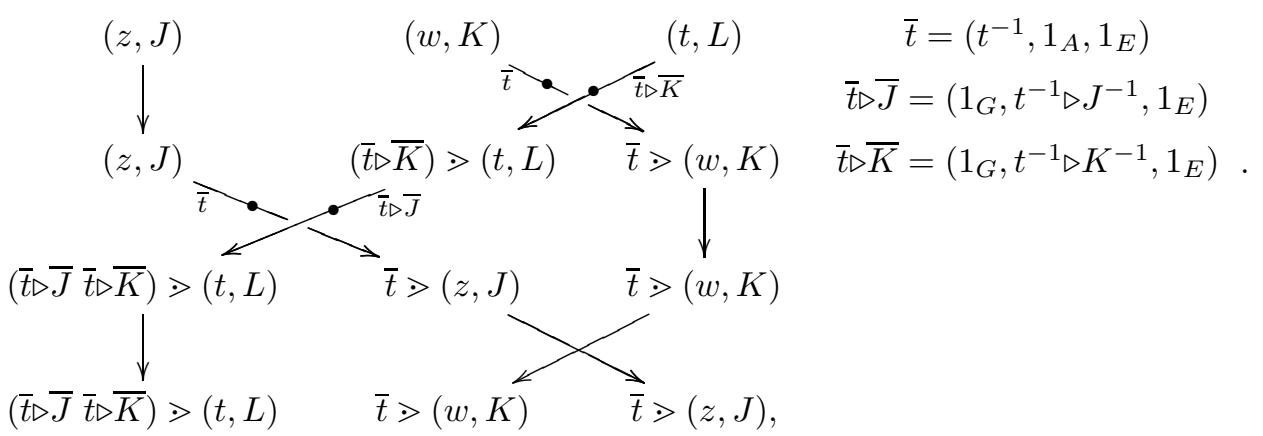


Morphisms (93) and (94) in TRANS $\left(S^{2}(\mathcal{G})\right)^{3} \rtimes \Sigma_{3}$ coincide, for each $(z, J),(w, K),(t, L) \in S^{2}(\mathcal{G})$. This follows since $\bar{t} \triangleright \bar{J} \bar{t} \triangleright \bar{K}=\bar{t} \triangleright \bar{K} \bar{t} \triangleright \bar{J}$, as the group operation in $A=\operatorname{ker}(\partial)$ is commutative; see Lem. 43.

Remark 60. Let $\mathcal{G}=(\partial: E \rightarrow G, \triangleright)$ be a crossed module. We have an abelian gr-group $(G, A=\operatorname{ker}(\partial))$ [6.2]. Write $A$ in multiplicative notation. The W-bikoid $X_{g r^{*}}^{+}$is obtained from the W-bikoid $X_{g r}^{+}$in (69), and vice versa. We have an inclusion morphism of groupoids $\operatorname{Inc}: \operatorname{TRANS}(G, A) \rightarrow \operatorname{TRANS}\left(S^{2}(\mathcal{G})\right)$, with:

$$
\operatorname{Inc}\left((h, K) \stackrel{(g, J)}{\longrightarrow}(g, J)(h, K)(g, J)^{-1}\right)=\left((h, K) \stackrel{\left(g, J, 1_{E}\right)}{\longrightarrow}\left(g, J, 1_{E}\right)>(h, K)\right) .
$$

This inclusion is bijective on objects. And then $X_{g r^{*}}^{+}$is obtained by transporting [6.1] $X_{g r}^{+}$along Inc.

In particular, 4.5 .1 gives a topological interpretation for the existence of the W-bikoid $X_{g r^{*}}^{+}$.

Remark 61. (Cf. [6.2] 6.3] and Rem. 60.) The representations of $\mathrm{WB}_{n}$ derived from $X_{g r^{*}}^{+}$do not appear to encode more information that those derived from $X_{g r}^{+}$. The extra degree of structure appearing in the underpinning groupoid $\operatorname{TRANS}\left(S^{2}(\mathcal{G})\right)$ of $X_{g r^{*}}^{+}$is [2.23] related to gauge transformations betwen 2connections. Hence $X_{g r^{*}}^{+}$is likely more fundamental than $X_{g r}^{+}$. From a strictly mathematical sense, this extra degree of freedom that $\operatorname{TRANS}\left(S^{2}(\mathcal{G})\right)$ has in comparison with $\operatorname{TRANS}(G, A)$ is essential when addressing invariants of welded knots derived from crossed modules, by using $X_{g r^{*}}^{+}$. This will be addressed in [40].

Remark 62. Let $\mathcal{G}=(\partial: E \rightarrow G, \triangleright)$ be a crossed module. The underlying W-birack (90) of $X_{g r^{*}}^{+}$is studied in [32. In the cases in Examples 31] and 34, this W-birack yields non-trivial invariants of welded knots that see beyond their knot groups; see also [60. This indicates that the representations of $\mathrm{WB}_{n}$ derived from $\mathcal{G}=(\partial: E \rightarrow G, \triangleright)$ carry more topological information than those derived (see [1.11] [1.12]) from $G$ alone.

Example 63. Continuing Rem. 62, Consider the crossed module $\mathcal{G}\left(\mathbb{Z}_{2}, \mathbb{Z}_{3}\right)$ derived [6.3] from the abelian

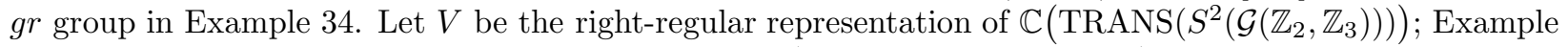
7. Let $V_{\text {OBJ }}$ be the object-regular representation of $\mathbb{C}\left(\operatorname{TRANS}\left(S^{2}\left(\mathcal{G}\left(\mathbb{Z}_{2}, \mathbb{Z}_{3}\right)\right)\right)\right)$; see Example 8 Let also $U$ and $U_{\text {OBJ }}$ be the right-regular and object-regular representations of $\mathbb{C}\left(\operatorname{AUT}\left(\mathbb{Z}_{2}\right)\right)$; see Example 3 . Consider the representations of $\mathrm{WB}_{2}$ derived from the W-bikoid $X_{g r *}^{+}$in (88), on $V \otimes V$ and on $V_{\mathrm{OBJ}} \otimes V_{\mathrm{OBJ}}$; see Thm. 38 or Thm. 41 . Consider the representations of $\mathrm{WB}_{2}$ on $U \otimes U$ and on $U_{\text {OBJ }} \otimes U_{\text {OBJ }}$, derived from the W-bikoid $X_{\mathbb{Z}_{2}}^{+}$in (65). The degree of $(-) \triangleleft^{*} S_{1}^{+}[2]$ in (76), induced by the braid generator $S_{1}^{+}[2]$ in (44) is:

$$
\begin{array}{ll}
2 \text { for the action of } \mathrm{WB}_{2} \text { on } U_{\mathrm{OBJ}} \otimes U_{\mathrm{OBJ}}, & 4 \text { for the action of } \mathrm{WB}_{2} \text { on } U \otimes U, \\
12 \text { for the action of } \mathrm{WB}_{2} \text { on } V_{\mathrm{OBJ}} \otimes V_{\mathrm{OBJ}}, & 12 \text { for the action of } \mathrm{WB}_{2} \text { on } V \otimes V .
\end{array}
$$

Hence Equation (95) tell us that the representations of $\mathrm{WB}_{n}$ derived from a W-bikoid may carry more information than those derived from its underlying W-birack [5.1] Comparing (951) and (96) gives an example of a case when the representations of $\mathrm{WB}_{n}$ derived from a crossed module $(\partial: G \rightarrow E, \triangleright)$ - in this case $\mathcal{G}\left(\mathbb{Z}_{2}, \mathbb{Z}_{3}\right)$ - are finner than those derived from the W-bikoid associated to $G$ alone.

\subsubsection{A W-bikoid structure in $\operatorname{TRANS}\left(T_{R}^{2}(\mathcal{G})\right)$}

Let $\mathcal{G}=(\partial: E \rightarrow G, \triangleright)$ be a crossed module. Let $R \in E$. (The physical meaning of $R$ is explained in [2.18] $)$ We now describe the W-bikoid $X_{R}^{+}$mentioned in the end of $\$ 2.3$ (Hence $X_{R}^{+}$is related to loop-particles in topological higher gauge theory.) This $X_{R}^{+}$is derived from and is isomorphic to $X_{g r^{*}}^{+}$in Equation (88).

Cf. Defs. 54, 55, and Lem. 56. There is a groupoid isomorphism $\Phi_{R}: \operatorname{TRANS}\left(S^{2}(\mathcal{G})\right) \rightarrow \operatorname{TRANS}\left(T_{R}^{2}(\mathcal{G})\right)$.

Theorem 64. We have a $W$-bikoid defined in the groupoid $X_{R}^{+}$in $\operatorname{TRANS}\left(T_{R}^{2}(\mathcal{G})\right.$ ). This $X_{R}^{+}$is obtained by transporting [6.1] $\left(\operatorname{TRANS}\left(S^{2}(\mathcal{G})\right), X_{g r^{*}}^{2}\right)$ in (88) to TRANS $\left(T_{R}^{2}(\mathcal{G})\right)$ along $\Phi_{R}$. The explicit form of $X_{R}^{+}$is 
(recalling the notation of Defs. 48 and 49 , and that we sometimes use $\overline{(-)}$ to denote inverses in a group):

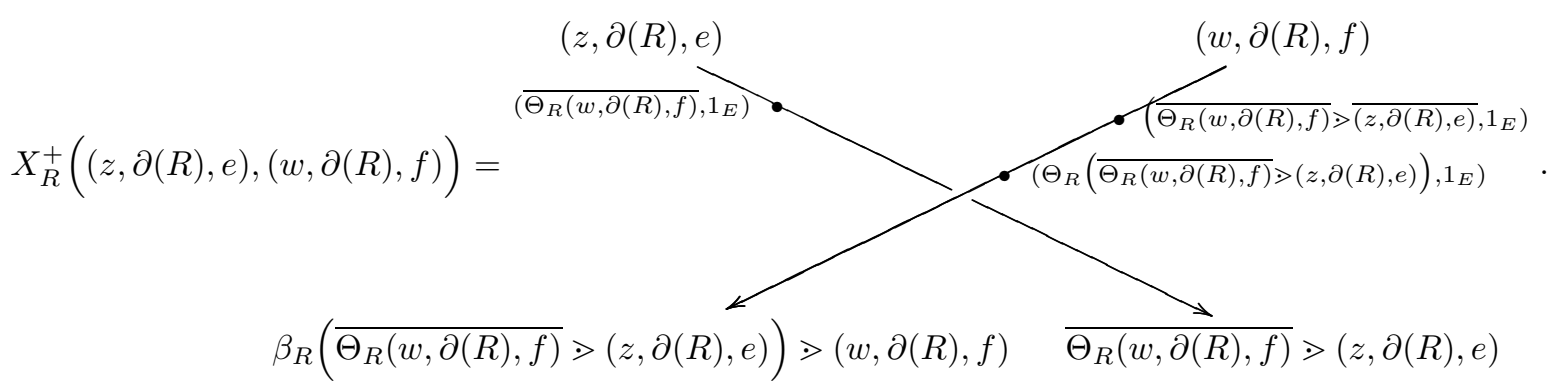

(Recall [6.11]]) Or, in another form:

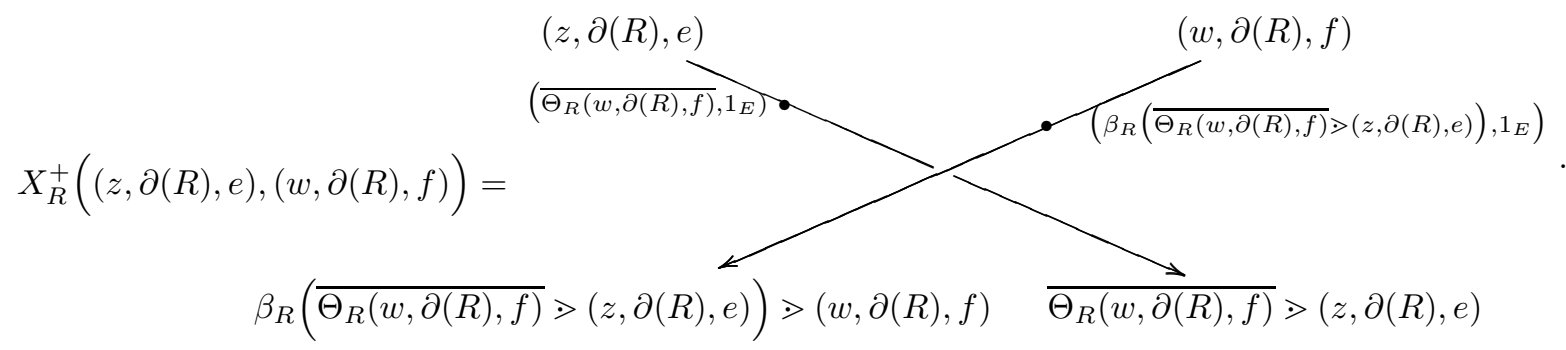

Explicitly, on the second strand of (97), we have the following element of $T_{R}^{2}(\mathcal{G})$ (see (82) and (83)):

$$
\begin{aligned}
\Theta_{R}\left(\overline{\Theta_{R}(w, \partial(R), f)} \gg(z, \partial(R), e)\right) & \overline{\Theta_{R}(w, \partial(R), f)} \triangleright \overline{(z, \partial(R), e)} \\
& =\beta_{R}\left(\overline{\Theta_{R}(w, \partial(R), f)} \gg(z, \partial(R), e)\right) \\
& =\beta_{R}\left(w^{-1} z w, \partial(R), R w^{-1} \triangleright R^{-1} w^{-1} \triangleright e\left(w^{-1} z\right) \triangleright R\left(w^{-1} z w\right) \triangleright R^{-1}\right) \\
& =\left(1, \partial(R), R\left(w^{-1} z\right) \triangleright R^{-1} w^{-1} \triangleright e^{-1} w^{-1} \triangleright R R^{-1}\right) .
\end{aligned}
$$

Hence the element of $T_{R}^{2}(\mathcal{G})$ associated to the target of the second strand of the diagram in (97) is:

$$
\begin{aligned}
\left(\beta _ { R } \left(\overline{\Theta_{R}(w, \partial(R), f)}\right.\right. & \left.>(z, \partial(R), e)), 1_{E}\right) \gg(w, \partial(R), f) \\
& =\left(w, \partial(R), R\left(w^{-1} z\right) \triangleright R^{-1} w^{-1} \triangleright e^{-1} w^{-1} \triangleright R R^{-1} f w \triangleright R R^{-1} \text { e } z \triangleright R w \triangleright R^{-1}\right) .
\end{aligned}
$$

And, on the first strand of the diagram in (97), we have the following element of $T_{R}^{2}(\mathcal{G})$ :

$$
\overline{\Theta_{R}(w, \partial(R), f)}=\left(w^{-1}, \partial(R), R w^{-1} \triangleright R^{-1}\right) .
$$

Hence the element of $T_{R}^{2}(\mathcal{G})$ associated to the target of the first strand in (97) is:

$$
\overline{\Theta_{R}(w, \partial(R), f)} \gg(z, \partial(R), e)=\left(w^{-1} z w, \partial(R), R w^{-1} \triangleright R^{-1} w^{-1} \triangleright e\left(w^{-1} z\right) \triangleright R\left(w^{-1} z w\right) \triangleright R^{-1}\right) .
$$

Remark 65. A more compact formulation of $X_{R}^{+}$in Equations (98) and (99) is in Equation (39).

Proof. By using (83), it follows that the formulations for $X_{R}^{+}$in (97) and (98) coincide, and it also follows (99). Equation (100) follows from (86). Analogously, (101) and (102) follow from Lem. 48 and (86).

We prove that $X_{R}^{+}$in (98) is obtained by transporting the bikoid $X_{g r^{*}}^{+}$in (88) along $\Phi_{R}$ : $\operatorname{TRANS}\left(S^{2}(\mathcal{G})\right) \rightarrow$ $\operatorname{TRANS}\left(T_{R}^{2}(\mathcal{G})\right)$ in Lem. [56, recall [6.1] We use the formulation in Equation (89) for $X_{g r^{*}}^{2}$. Recall Defs. 47 and 52. That $X_{R}^{+}$in (97) is obtained by transporting $X_{g r^{*}}^{+}$in (89) along $\Phi_{R}$ follows swiftly from the construction of $\Phi_{R}$ : $\operatorname{TRANS}\left(S^{2}(\mathcal{G})\right) \rightarrow \operatorname{TRANS}\left(T_{R}^{2}(\mathcal{G})\right)$ in Lem. [56, together with Lem. 48, 50 and 6.8] 


\section{References}

[1] K. Walker and Z. Wang, "(3+ 1)-TQFTs and topological insulators," Front. Phys., vol. 7, no. 2, pp. 150$159,2012$.

[2] Z. Kádár, P. Martin, E. Rowell, and Z. Wang, "Local representations of the loop braid group," Glasg. Math. J., vol. 59, no. 2, pp. 359-378, 2017.

[3] C.-H. Lin and M. Levin, "Loop braiding statistics in exactly soluble three-dimensional lattice models," Phys. Rev. B, vol. 92, p. 035115, Jul 2015.

[4] C. Wang and M. Levin, "Braiding statistics of loop excitations in three dimensions," Phys. Rev. Lett., vol. 113, p. 080403, Aug 2014.

[5] D. V. Else and C. Nayak, "Cheshire charge in (3+1)-dimensional topological phases," Phys. Rev. B, vol. 96, p. 045136, Jul 2017.

[6] C. W. von Keyserlingk, F. J. Burnell, and S. H. Simon, "Three-dimensional topological lattice models with surface anyons," Phys. Rev. B, vol. 87, p. 045107, Jan 2013.

[7] A. Bullivant, M. Calçada, Z. Kádár, J. Faria Martins, and P. Martin, "Topological phases from higher gauge symmetry in 3+1 dimensions," Phys. Rev. B, vol. 95, p. 155118, Apr 2017.

[8] A. Bullivant, M. Calcada, Z. Kádár, J. Faria Martins, and P. Martin, "Higher lattices, discrete two-dimensional holonomy and topological phases in $(3+1)$ D with higher gauge symmetry," ArXiv: 1702.00868, Feb. 2017.

[9] A. Kapustin and R. Thorngren, "Higher symmetry and gapped phases of gauge theories," ArXiv: 1309.4721, Sept. 2013.

[10] D. J. Williamson and Z. Wang, "Hamiltonian models for topological phases of matter in three spatial dimensions," Annals of Physics, vol. 377, pp. 311 - 344, 2017.

[11] J. C. Baez and J. Huerta, "An invitation to higher gauge theory," General Relativity and Gravitation, vol. 43, no. 9, pp. 2335-2392, 2011.

[12] J. C. Baez and U. Schreiber, "Higher gauge theory," in Categories in algebra, geometry and mathematical physics. Conference and workshop in honor of Ross Street's 60th birthday, Sydney and Canberra, Australia, July 11-16/July 18-21, 2005, pp. 7-30, Providence, RI: American Mathematical Society (AMS), 2007.

[13] J. C. Baez, D. K. Wise, and A. S. Crans, "Exotic statistics for strings in 4D BF theory," Adv. Theor. Math. Phys., vol. 11, no. 5, pp. 707-749, 2007.

[14] C. Damiani, "A journey through loop braid groups," Expositiones Mathematicae, vol. 35, no. 3, pp. 252 $-285,2017$.

[15] D. L. Goldsmith, "The theory of motion groups," Michigan Math. J., vol. 28, no. 1, pp. 3-17, 1981.

[16] J. K. Pachos, Introduction to topological quantum computation. Cambridge University Press, 2012.

[17] R. Fenn, R. Rimányi, and C. Rourke, "The braid-permutation group," Topology, vol. 36, no. 1, pp. 123$135,1997$.

[18] S. Kamada, "Braid presentation of virtual knots and welded knots," Osaka J. Math., vol. 44, no. 2, pp. $441-458,2007$.

[19] L. H. Kauffman and S. Lambropolou, "Virtual braids and the L-move," Journal of Knot Theory and Its Ramifications, vol. 15, no. 06, pp. 773-811, 2006.

[20] L. H. Kauffman, "Virtual knot theory," European J. Combin., vol. 20, no. 7, pp. 663-690, 1999. 
[21] V. G. Bardakov, P. Bellingeri, and C. Damiani, "Unrestricted virtual braids, fused links and other quotients of virtual braid groups," Journal of Knot Theory and Its Ramifications, vol. 24, no. 12, p. $1550063,2015$.

[22] A. Bartholomew and R. Fenn, "Biquandles of small size and some invariants of virtual and welded knots," J. Knot Theory Ramifications, vol. 20, no. 7, pp. 943-954, 2011.

[23] V. O. Manturov, "Recognition of virtual braids," Journal of Mathematical Sciences, vol. 131, pp. 54095419 , Nov 2005.

[24] C. Kassel, Quantum groups. New York, NY: Springer-Verlag, 1995.

[25] A. Brochier, "Virtual tangles and fiber functors," ArXiv: 1602.03080, Feb. 2016.

[26] R. Fenn, M. Jordan-Santana, and L. Kauffman, "Biquandles and virtual links," Topology Appl., vol. 145, no. 1-3, pp. 157-175, 2004.

[27] R. Bauernschmidt and S. Nelson, "Birack modules and their link invariants," Commun. Contemp. Math., vol. 15, no. 3, pp. 1350006, 13, 2013.

[28] M. S. J. Scott Carter, Mohamed Elhamdadi, "Homology theory for the set-theoretic Yang-Baxter equation and knot invariants from generalizations of quandles," Fundamenta Mathematicae, vol. 184, no. 1, pp. 31-54, 2004.

[29] R. Fenn, D. P. Ilyutko, L. H. Kauffman, and V. O. Manturov, "Unsolved problems in virtual knot theory and combinatorial knot theory," in Knots in Poland III. Part III, vol. 103 of Banach Center Publ., pp. 9-61, Polish Acad. Sci. Inst. Math., Warsaw, 2014.

[30] V. Manturov, Knot theory. Chapman \& Hall/CRC, Boca Raton, FL, 2004.

[31] M. Elhamdadi and S. Nelson, Quandles: an Introduction to the Algebra of Knots. Student Mathematical Library, American Mathematical Society, 2015.

[32] L. H. Kauffman and J. Faria Martins, "Invariants of welded virtual knots via crossed module invariants of knotted surfaces," Compos. Math., vol. 144, no. 4, pp. 1046-1080, 2008.

[33] B. Audoux, "On the welded tube map," in Knot theory and its applications, vol. 670 of Contemp. Math., pp. 261-284, Amer. Math. Soc., Providence, RI, 2016.

[34] V. G. Bardakov and P. Bellingeri, "Groups of virtual and welded links," J. Knot Theory Ramifications, vol. 23, no. 3, pp. 1450014, 23, 2014.

[35] J. S. Carter, "A survey of quandle ideas," in Introductory lectures on knot theory, vol. 46 of Ser. Knots Everything, pp. 22-53, World Sci. Publ., Hackensack, NJ, 2012.

[36] J. S. Carter, D. Jelsovsky, S. Kamada, and M. Saito, "Computations of quandle cocycle invariants of knotted curves and surfaces," Adv. Math., vol. 157, no. 1, pp. 36-94, 2001.

[37] J. S. Carter, D. Jelsovsky, S. Kamada, L. Langford, and M. Saito, "State-sum invariants of knotted curves and surfaces from quandle cohomology," Electron. Res. Announc. Amer. Math. Soc., vol. 5, pp. 146-156, 1999.

[38] M. Eisermann, "Knot colouring polynomials," Pac. J. Math., vol. 231, no. 2, pp. 305-336, 2007.

[39] B. Winter, "The classification of spun torus knots," J. Knot Theory Ramifications, vol. 18, no. 9, pp. $1287-1298,2009$.

[40] J. Faria Martins et al, "Invariants of welded knots derived from finite crossed modules," In preparation.

[41] P. Higgins, "Categories and groupoids," Repr. Theory Appl. Categ., vol. 2005, no. 7, pp. 1-195, 2005.

[42] F. Bais, "Flux metamorphosis," Nuclear Physics B, vol. 170, no. 1, pp. 32 - 43, 1980. 
[43] F. A. Bais, P. van Driel, and M. de Wild Propitius, "Quantum symmetries in discrete gauge theories," Physics Letters B, vol. 280, no. 1, pp. 63 - 70, 1992.

[44] M. de Wild Propitius and F. A. Bais, Discrete Gauge Theories, pp. 353-439. New York, NY: Springer New York, 1999.

[45] A. F. Bais, B. J. Schroers, and J. K. Slingerland, "Hopf symmetry breaking and confinement in (2+1)dimensional gauge theory," Journal of High Energy Physics, vol. 2003, no. 05, p. 068, 2003.

[46] M. Dokuchaev, R. Exel, and P. Piccione, "Partial representations and partial group algebras," Journal of Algebra, vol. 226, no. 1, pp. 505 - 532, 2000.

[47] S. Willerton, "The twisted drinfeld double of a finite group via gerbes and finite groupoids," Algebraic E Geometric Topology, vol. 8, no. 3, pp. 1419-1457, 2008.

[48] J. C. Morton, "Two-vector spaces and groupoids," Applied Categorical Structures, vol. 19, pp. 659-707, Aug 2011.

[49] D. Altschuler and A. Coste, "Quasi-quantum groups, knots, three-manifolds, and topological field theory," Communications in Mathematical Physics, vol. 150, pp. 83-107, Nov 1992.

[50] V. G. Turaev, Quantum invariants of knots and 3-manifolds, vol. 18 of De Gruyter Studies in Mathematics. De Gruyter, Berlin, 2016.

[51] N. Reshetikhin and V. Turaev, "Ribbon graphs and their invariants derived from quantum groups," Commun. Math. Phys., vol. 127, no. 1, pp. 1-26, 1990.

[52] C. Mochon, "Anyons from nonsolvable finite groups are sufficient for universal quantum computation," Phys. Rev. A, vol. 67, p. 022315, Feb 2003.

[53] C. Delcamp, B. Dittrich, and A. Riello, "Fusion basis for lattice gauge theory and loop quantum gravity," Journal of High Energy Physics, vol. 2017, p. 61, Feb 2017.

[54] G. Böhm, F. Nill, and K. Szlachányi, "Weak Hopf algebras. I. Integral theory and $C^{*}$-structure," $J$. Algebra, vol. 221, no. 2, pp. 385-438, 1999.

[55] D. Nikshych, V. Turaev, and L. Vainerman, "Invariants of knots and 3-manifolds from quantum groupoids," in Proceedings of the Pacific Institute for the Mathematical Sciences Workshop "Invariants of Three-Manifolds" (Calgary, AB, 1999), vol. 127, pp. 91-123, 2003.

[56] P. Etingof, D. Nikshych, and V. Ostrik, "On fusion categories," Ann. of Math. (2), vol. 162, no. 2, pp. 581-642, 2005.

[57] D. N. Yetter, "TQFTs from homotopy 2-types," J. Knot Theory Ramifications, vol. 2, no. 1, pp. 113-123, 1993.

[58] J. Faria Martins and T. Porter, "On Yetter's invariant and an extension of the Dijkgraaf-Witten invariant to categorical groups," Theory Appl. Categ., vol. 18, pp. No. 4, 118-150, 2007.

[59] J. Faria Martins, "Categorical groups, knots and knotted surfaces," J. Knot Theory Ramifications, vol. 16, no. 9, pp. 1181-1217, 2007.

[60] J. Faria Martins, "The fundamental crossed module of the complement of a knotted surface," Trans. Amer. Math. Soc., vol. 361, no. 9, pp. 4593-4630, 2009.

[61] A. Y. Kitaev, "Fault-tolerant quantum computation by anyons," Annals of Physics, vol. 303, no. 1, pp. $2-30,2003$.

[62] M. Benini, A. Schenkel, and R. J. Szabo, "Homotopy colimits and global observables in abelian gauge theory," Lett. Math. Phys., vol. 105, no. 9, pp. 1193-1222, 2015. 
[63] K. Costello, Renormalization and effective field theory, vol. 170 of Mathematical Surveys and Monographs. American Mathematical Society, Providence, RI, 2011.

[64] T. Lan and X.-G. Wen, "Topological quasiparticles and the holographic bulk-edge relation in $(2+1)$ dimensional string-net models," Phys. Rev. B, vol. 90, p. 115119, Sep 2014.

[65] C. Nayak, S. H. Simon, A. Stern, M. Freedman, and S. Das Sarma, "Non-abelian anyons and topological quantum computation," Rev. Mod. Phys., vol. 80, pp. 1083-1159, Sep 2008.

[66] J. S. Birman, Braids, links, and mapping class groups. Princeton University Press, Princeton, N.J.; University of Tokyo Press, Tokyo, 1974. Annals of Mathematics Studies, No. 82.

[67] T. E. Brendle and A. Hatcher, "Configuration spaces of rings and wickets," Comment. Math. Helv., vol. 88, no. 1, pp. 131-162, 2013.

[68] U. Schreiber and K. Waldorf, "Connections on non-abelian gerbes and their holonomy," Theory Appl. Categ., vol. 28, pp. 476-540, 2013.

[69] U. Schreiber and K. Waldorf, "Smooth functors vs. differential forms," Homology Homotopy Appl., vol. 13, no. 1, pp. 143-203, 2011.

[70] J. Faria Martins and R. Picken, "Surface holonomy for non-Abelian 2-bundles via double groupoids," Adv. Math., vol. 226, no. 4, pp. 3309-3366, 2011.

[71] R. Brown, P. J. Higgins, and R. Sivera, Nonabelian algebraic topology. Filtered spaces, crossed complexes, cubical homotopy groupoids. With contributions by Christopher D. Wensley and Sergei V. Soloviev. Zürich: European Mathematical Society (EMS), 2011.

[72] C. Wockel, "Principal 2-bundles and their gauge 2-groups," Forum Math., vol. 23, no. 3, pp. 565-610, 2011.

[73] R. Brown, "Groupoids and crossed objects in algebraic topology," Homology Homotopy Appl., vol. 1, pp. 1-78, 1999.

[74] J. C. Baez and A. D. Lauda, "Higher-dimensional algebra. V: 2-Groups," Theory Appl. Categ., vol. 12, pp. 423-491, 2004.

[75] J. C. Morton and R. Picken, "Transformation double categories associated to 2-group actions," Theory Appl. Categ., vol. 30, pp. 1429-1468, 2015.

[76] S. Mac Lane, Categories for the working mathematician. Springer, second ed., 1998.

[77] M. Gould, "Quantum double finite group algebras and their representations," Bull. Aust. Math. Soc., vol. 48, no. 2, pp. 275-301, 1993.

[78] G. Burde, H. Zieschang, and M. Heusener, Knots. 3rd fully revised and extented edition., vol. 5. Berlin: Walter de Gruyter, 3rd fully revised and extented edition ed., 2014.

[79] A. Kawauchi, A survey of knot theory. Transl. from the Japan. by the author. Basel: Birkhäuser, 1996.

[80] C. Rourke and B. Sanderson, Introduction to piecewise-linear topology. Ergebnisse der Mathematik und ihrer Grenzgebiete, Springer-Verlag, 1972.

[81] T. Kanenobu, "Forbidden moves unknot a virtual knot," Journal of Knot Theory and Its Ramifications, vol. 10, no. 01, pp. 89-96, 2001.

[82] S. Nelson, "Unknotting virtual knots with Gauss diagram forbidden moves," J. Knot Theory Ramifications, vol. 10, no. 6, pp. 931-935, 2001.

[83] T. Nasybullov, "Classification of fused links," J. Knot Theory Ramifications, vol. 25, no. 14, pp. 1650076, $21,2016$. 
[84] J. Wilson, "Representation stability for the cohomology of the pure string motion groups," Algebr. Geom. Topol., vol. 12, no. 2, pp. 909-931, 2012.

[85] A. G. Savushkina, "On the group of conjugating automorphisms of a free group," Mathematical Notes, vol. 60 , no. 1 , pp. $68-80,1996$.

[86] P. P. Martin, D. Woodcock, and D. Levy, "A diagrammatic approach to Hecke algebras of the reflection equation," Journal of Physics A: Mathematical and General, vol. 33, no. 6, p. 1265, 2000.

[87] S. Nelson and J. L. Rische, "On bilinear biquandles," Colloq. Math., vol. 112, no. 2, pp. 279-289, 2008.

[88] D. Stanovský, "On axioms of biquandles," J. Knot Theory Ramifications, vol. 15, no. 7, pp. 931-933, 2006.

[89] S. Satoh, "Virtual knot presentation of ribbon torus-knots," J. Knot Theory Ramifications, vol. 9, no. 4, pp. $531-542,2000$.

[90] D. Rolfsen, Knots and links, vol. 7 of Mathematics Lecture Series. Publish or Perish, Inc., Houston, TX, 1990. Corrected reprint of the 1976 original.

[91] A. Hatcher, Algebraic topology. Cambridge University Press, Cambridge.

[92] D. Bar-Natan, "Balloons and hoops and their universal finite-type invariant, BF theory, and an ultimate Alexander invariant," Acta Math. Vietnam., vol. 40, no. 2, pp. 271-329, 2015. 